Apresentações dos grupos de tranças em superfícies

\author{
Juliana Roberta Theodoro de Lima
}


SERVIÇO DE PÓS-GRADUAÇÃO DO ICMC-USP

Data de Depósito:

Assinatura:

\title{
Apresentações dos grupos de tranças em superfícies
}

\author{
Juliana Roberta Theodoro de Lima
}

Orientador: Prof. Dr. José Eduardo Prado Pires de Campos

Dissertação apresentada ao Instituto de Ciências Matemáticas e de Computação - ICMC-USP, como parte dos requisitos para obtenção do título de Mestre em Ciências - Matemática.

USP - São Carlos

Maio/2010 

"É melhor atirar-se à luta em busca de dias melhores, mesmo correndo o risco de perder tudo, do que permanecer estático, como os pobres de espírito, que não lutam, mas também não vencem, que não conhecem a dor da derrota, nem a glória de ressurgir dos escombros. Esses pobres de espírito, ao final de sua jornada na Terra não agradecem a Deus por terem vivido, mas desculpam-se perante Ele, por terem apenas passado pela vida."

Bob Marley 


\section{Agradecimentos}

Gostaria de começar agradecendo à Deus, que sempre me deu forças para conseguir todas as graças já alcançadas. À minha família e, em especial, à pessoa que sempre esteve presente nos momentos mais importantes da minha vida, me apoiando e dizendo pra eu erguer a cabeça e seguir em frente sempre que precisei: pai, se tenho vontade de ser alguém e de querer que minha vida valha a pena é porque tenho o senhor, meu exemplo.

Para o meu super namorado, melhor amigo, confidente e "personal" monitor, Matheus, que está comigo em todos os instantes, nos bons e nos ruins, nas longas horas de estudo, pelas broncas quando preciso, sempre me fazendo enxergar a vida de um modo mais simples e mais maravilhoso...te amo demais!!!

Agradeço à todos os professores, em especial ao meu orientador prof. Pires que estava pronto para sanar minhas dúvidas sempre que precisei, com muita paciência e dedicação; aos prof. Denise e Edivaldo pela amizade, atenção nas disciplinas, fazendo com que eu me apaixone cada vez mais pela Topologia Algébrica; à prof. Márcia pela amizade e pelos puxões de orelha nos corredores do ICMC sempre preocupada com o melhor para todos.

Às minhas grandes amigas de longa data Lidiane B. de Souza, Aryane Tofanello e Luana Caetano pelas lágrimas, risadas, baladas e histórias engraçadas. Aos amigos que fiz a pouco, mas que já moram no meu coração: Moreno Bonucci, Rodrigo P. Brum, Rafael Rossato, Alex Rezende, Thaís Dalbelo, Renato Mollo, Jaqueline Godoy e Iris de Oliveira pelos passeios, reuniões de estudo, troca de listas de exercícios e deliciosos almoços. Adoro vocês!

Agradeço à CAPES- Coordenação de Aperfeiçoamento de Pessoal de Nível Superior pela bolsa de mestrado e à FAPESP- Fundação de Amparo a Pesquisa do Estado de São Paulo, projeto temático número: 08/57607-6.

E por fim, fazendo minhas as palavras de Chico Xavier: não posso deixar de agradecer aos meus obstáculos; se não fosse por eles, eu não teria saído do lugar. Um caminho sem 
obstáculos nos impede de caminhar. 


\section{Resumo}

Neste trabalho, estudamos os grupos de tranças em superfícies visando encontrar apresentações para estes grupos em superfícies fechadas orientáveis de gênero $g \geq 1$ ou superfícies fechadas não orientáveis de gênero $g \geq 2$. Uma consequência destas apresentações é resolvermos o problema da palavra, que consiste em encontrar um algoritmo para decidir quando uma dada palavra num grupo definido por seus geradores e suas relações é a palavra trivial. 


\section{Abstract}

In this work, we find presentations for surface braid groups either in closed orientable surfaces of genus $g \geq 1$ or in closed non-orientable surfaces of genus $g \geq 2$. A consequence of this presentations is to solve the word problem, which consists in finding an algorithm to decide when a given word in a group defined by its generators and its relations is the trivial word. 


\section{Sumário}

Introdução 1

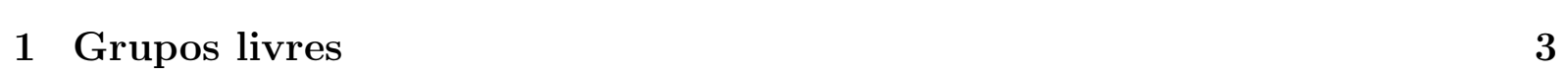

1.1 Introducão . . . . . . . . . . . . . . . . . . . . . . . . . . 3

1.2 Geradores e relatores . . . . . . . . . . . . . . . . . . 11

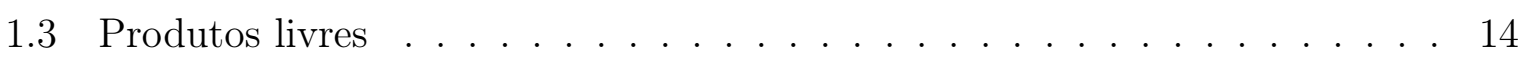

1.4 Push-outs e produtos livres amalgamados . . . . . . . . . . . . . . 15

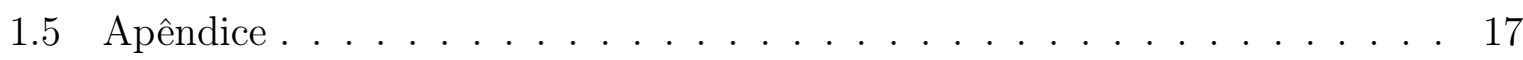

1.5.1 O problema da palavra . . . . . . . . . . . . . . 17

1.5.2 O algoritmo de Dehn . . . . . . . . . . . . . . . 17

2 Apresentacões de produtos diretos, semidiretos e extensões de grupos 19

2.1 Apresentação de produtos diretos . . . . . . . . . . . . . . . . . . . . . . . . 19

2.2 Produtos semidiretos . . . . . . . . . . . . . . . . . . . . 20

2.3 Apresentação de extensões de grupos . . . . . . . . . . . . . . . . . . . . . 21

2.3 .1 Conceitos básicos . . . . . . . . . . . . . . . . . . . 21

2.3 .2 O teorema principal . . . . . . . . . . . . . . . . 24

2.3 .3 Casos especiais . . . . . . . . . . . . . . . . 25

3 Estrutura do grupo fundamental de uma superfície compacta 29

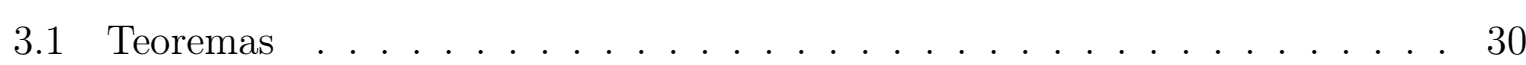

3.2 cálculo de grupos fundamentais . . . . . . . . . . . . . . . . . . . 31

3.2.1 O toro 2-dimensional $\mathbf{T} \ldots \ldots \ldots$. . . . . . . . . . 31

3.2 .2 Soma conexa de n-toros . . . . . . . . . . . . . . . . . . 33

3.3 Cálculo de grupos fundamentais . . . . . . . . . . . . . . . . . . . . . . . . 34

3.3.1 O plano real projetivo $P_{2}(\mathbb{R}) \ldots \ldots \ldots . \ldots . \ldots . \ldots 34$ 
3.3.2 Soma conexa de n-planos projetivos . . . . . . . . . . . . . . . 35

4 Introducão aos grupos das tranças no disco $\quad 37$

4.1 Tranças Geométricas . . . . . . . . . . . . . . . . . . . . . 37

5 Apresentacões dos grupos de tranças em superfícies 45

5.1 Introducão . . . . . . . . . . . . . . . . . . . . . . . 45

5.2 Interpretacões geométricas e afirmações . . . . . . . . . . . . . . . . . . 47

5.2 .1 Caso Orientável . . . . . . . . . . . . . . . . . . 47

5.2 .2 Caso não-orientável . . . . . . . . . . . . . . . . . . . . 53

5.3 As ideias chave das demonstracões . . . . . . . . . . . . . . . . . . 57

5.4 O grupo de trancas de uma superfície orientável . . . . . . . . . . . . . . . 59

5.5 O grupo de trancas de uma superfície não orientável . . . . . . . . . . . . . 88

5.6 O problema da palavra . . . . . . . . . . . . . . . . . . . . 111

5.7 Apêndice . . . . . . . . . . . . . . . . . . . . 115 


\section{Introdução}

Em 1925, Artin introduziu o estudo de tranças (braids), o qual se relaciona profundamente com o estudo de nós e enlaçamentos (links). Um resultado importante obtido por Artin foi os seu teorema da apresentação que fornece uma apresentação para o grupo das tranças no disco.

A teoria de tranças se desenvolveu em várias direções com os trabalhos de Alexander, Markov, Birman e outros. A teoria básica pode ser encontrada em [B].

Mais recentemente, uma das áreas de pesquisa que tem se desenvolvido é o grupo de tranças em superfícies.

Neste trabalho, o principal objetivo é estudar apresentações do grupo das tranças e do grupo das tranças puras em uma superfície fechada. Para tanto, usaremos como referência principal o artigo [GM]. A fim de compreendê-lo utilizaremos alguns dos resultados clássicos sobre tranças ([B], [LH] e [BZ]) bem como um pouco da teoria combinatória de grupos ([C] e [LS]).

Para finalizar, aproveitando esta introdução, vamos dar uma breve referência das figuras que foram extraídas de alguns textos: no Capítulo 3 deste trabalho, as figuras são encontradas em [M]; as figuras do Capítulo 4 são encontradas em [B], [LH] e, por último, as figuras do capítulo 5 são encontradas em [GM]. 


\section{Capítulo}

1

\section{Grupos livres}

\subsection{Introdução}

Seja X um subconjunto gerador de um grupo G. Certos produtos de elementos de $\mathrm{X}$ e seus inversos serão 1 para quaisquer X e G; por exemplo, $x y y z^{-1} z y^{-1} y^{-1} x^{-1}$. Outros produtos tais como $x y z$ ou $x x$ só serão 1 para algumas escolhas de $\mathrm{X}$ e $\mathrm{G}$ mas não para outras.

Estes pares G e X para os quais um produto de elementos em $X \cup X^{-1}$ é 1 somente quando as propriedades que valem em todos os grupos (associatividade, elemento neutro e simétrico) exigirem que este produto seja 1 são de nosso interesse. O grupo G será chamado de grupo livre. Veremos a definição formal a seguir:

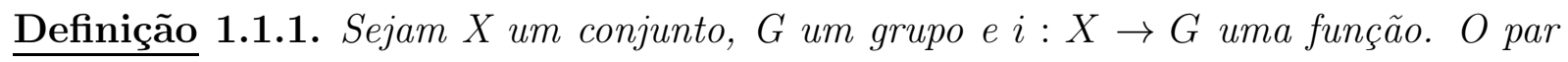
$(G, i)$ é chamado livre sobre $X$ se para cada grupo $H$ e para cada função $f: X \rightarrow H$ existir um único homomorfismo $\varphi: G \rightarrow H$ tal que $f=\varphi \circ i$.

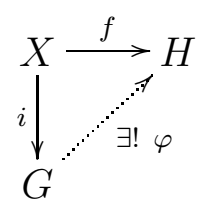

Em particular, o grupo trivial é livre sobre o conjunto vazio e o grupo cíclico infinito $\mathbb{Z}$ é livre em um conjunto unitário $\{x\}$, sendo $i(x)=1$. 
Se $(G, i)$ for livre sobre $X$ e $\varphi: G \rightarrow H$ for um isomorfismo então $(H, \varphi \circ i)$ será livre sobre X.

A proposição a seguir é a recíproca desta propriedade:

Proposição 1.1.2. Sejam $\left(G_{1}, i_{1}\right)$ e $\left(G_{2}, i_{2}\right)$ livres sobre $X$. Então existe um isomorfismo $\varphi: G_{1} \rightarrow G_{2}$ tal que $\varphi \circ i_{1}=i_{2}$.

Demonstração: Pela hipótese, $\left(G_{1}, i_{1}\right)$ é livre sobre X. Logo, exite um único homomorfismo $\varphi: G_{1} \rightarrow G_{2}$ tal que $i_{2}=\varphi \circ i_{1}$. Da mesma forma, como $\left(G_{2}, i_{2}\right)$ é livre sobre X, existe um único homomorfismo $\psi: G_{2} \rightarrow G_{1}$ tal que $i_{1}=\psi \circ i_{2}$.

Assim, sendo $I_{1}: G_{1} \rightarrow G_{1}$ e $I_{2}: G_{2} \rightarrow G_{2}$ as identidades em $G_{1}$ e $G_{2}$ respectivamente, temos:

$$
I_{1} \circ i_{1}=i_{1}=\psi \circ i_{2}=\psi \circ \varphi \circ i_{1}=(\psi \circ \varphi) \circ i_{1}
$$

Pela unicidade dos diagramas, $I_{1}=\psi \circ \varphi$.

De maneira análoga, $I_{2}=\varphi \circ \psi$. Portanto, $\varphi$ é isomorfismo.

Proposição 1.1.3. Se $(F, i)$ é um grupo livre sobre $X$, então i é injetora.

Demonstração: Veja [C].

Agora, vamos construir um grupo livre sobre $X$, começando com uma construção auxiliar:

Sejam $X$ um conjunto qualquer e $M(X)$ o conjunto de todas as sequências finitas $\left(x_{i_{1}}, \ldots, x_{i_{n}}\right)$ de elementos de $X$, onde $n \geq 0$ (se $n=0$ então teremos a sequência vazia denotada por ()).

Portanto, $M(X)=\left\{\left(x_{i_{1}}, \ldots, x_{i_{n}}\right) ; x_{i_{k}} \in X, k=1, \ldots, n\right\}$. Vamos definir a multiplicação em $M(X)$ por:

$$
\left(x_{i_{1}}, \ldots, x_{i_{n}}\right) .\left(x_{j_{1}}, \ldots, x_{j_{m}}\right)=\left(x_{i_{1}}, \ldots, x_{i_{n}}, x_{j_{1}}, \ldots, x_{j_{m}}\right)
$$

Esta multiplicação é obviamente associativa e o elemento identidade é a sequência vazia que denotaremos por 1 . Observe que $X \rightarrow M(X)$ dada por $x \mapsto(x)$ é uma função injetora e, identificando $x \operatorname{com}(x)$, podemos escrever cada elemento de $M(X)$ de modo único como um produto $x_{i_{1}} \ldots x_{i_{n}}$, para algum $n$. Vamos chamar $M(X)$ de monóide livre de $X$. 
Seja $x_{i_{1}} \ldots x_{i_{n}}$ um elemento em $M(X)$. Um segmento de $x_{i_{1}} \ldots x_{i_{n}}$ é um elemento $x_{i_{r}} x_{i_{r+1}} \ldots x_{i_{s}}$, onde $1 \leq r \leq s \leq n$. Ele é dito segmento inicial se $r=1$, segmento final se $s=n$ e segmento próprio se $r \neq 1$ ou $s \neq n$.

Observação 1.1.4. Um ponto técnico é omitido nesta construção. Desde que X é um conjunto arbitrário, é possível que algum elemento de $X$ possa ser uma sequência finita de elementos de $X$. Queremos distinguir um elemento de $X$ de um elemento de $M(X)$ : o caminho mais simples para sanar este problema é substituir $X$ pelo conjunto $X^{\prime}$ onde:

$$
X^{\prime}=\{\{x\} ; x \in X\}
$$

e assim definimos o monóide livre $M\left(X^{\prime}\right)$.

Contudo, vamos ignorar este detalhe no futuro.

Agora, vamos construir o grupo livre sobre $X$ : vamos tomar o conjunto $\bar{X}$ bijetor com $X$ que leva $x$ em $\bar{x}$ e tal que $X \cap \bar{X}=\emptyset$. Usualmente, denotaremos $\bar{x}$ por $x^{-1}$ e $x^{1}$ ao invés de $x$. Os elementos de $M(X \cup \bar{X})$ são chamados palavras em $X$. Assim, temos:

$$
M(X \cup \bar{X})=\left\{x_{i_{1}}^{\xi_{1}} \ldots x_{i_{n}}^{\xi_{n}} ; x_{i_{k}}^{\xi_{k}} \in X \cup \bar{X}, k=1, \ldots, n\right\}
$$

Se $w$ for uma palavra $x_{i_{1}}^{\xi_{1}} \ldots x_{i_{n}}^{\xi_{n}}$ então $n$ será chamado comprimento de $w$, denotado por $|w|$ ou por $l(w)$, e cada elemento $x_{i_{k}}^{\xi_{k}}$ será chamado letra de $w$. Uma palavra $w$ é dita reduzida se, para cada $1 \leq r \leq n-1$ ou $i_{r+1} \neq i_{r}$ ou $i_{r+1}=i_{r} \operatorname{com} \xi_{r+1} \neq-\xi_{r}$. A palavra vazia é uma palavra reduzida.

Suponhamos que $w$ não seja uma palavra reduzida e vamos escolher $r$ tal que $i_{r+1}=i_{r}$ e $\xi_{r+1}=-\xi_{r}$. Seja $w^{\prime}$ a palavra obtida de $w$ retirando o par adjacente de letras $x_{i_{r}}^{\xi_{r}} \mathrm{e}$ $x_{i_{r+1}}^{\xi_{r+1}}$. Dizemos que $w^{\prime}$ foi obtida de $w$ através de uma redução elementar.

Se $w^{\prime \prime}$ for obtida de $w$ por uma sequência de reduções elementares então dizemos que $w^{\prime \prime}$ foi obtida de $w$ através de uma redução.

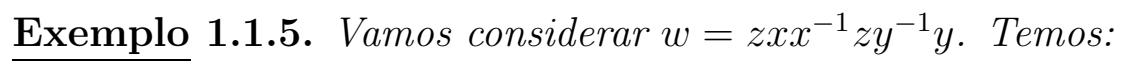

- w não é uma palavra reduzida;

- $w^{\prime}=z z y^{-1} y$ é obtida de $w$ através de uma redução elementar;

- $w^{\prime \prime}=z x x^{-1} z$ é obtida de $w$ através de uma redução elementar; 
- $w^{\prime \prime \prime}=z z$ é obtida de $w$ através de uma redução.

Dadas $w$ e $w^{\prime}$, vamos definir a seguinte relação: $w \approx w^{\prime}$ se, e somente se, $w$ é igual a $w^{\prime}$ ou existe uma sequência de palavras $w_{1}, \ldots, w_{k}$, para algum $k$, tal que $w_{1}=w, w_{k}=w^{\prime}$ e, para cada $j<k, w_{j+1}$ é obtido de $w_{j}$ (ou vice-versa) por redução elementar.

Claramente, $\approx$ é uma relação de equivalência. Vamos denotar o conjunto das classes de equivalência por $F(X)$ :

$$
F(X)=\{[w] ; w \text { é uma palavra em } M(X \cup \bar{X})\},[w]=\left\{w^{\prime} ; w \approx w^{\prime}\right\}
$$

É fácil ver que são válidas as seguintes propriedades:

1. Se $u, v, w$ e $w^{\prime}$ são palavras tal que $w \approx w^{\prime}$ então $u w v \approx u w^{\prime} v$.

2. Se $u \approx u^{\prime}$ e $w \approx w^{\prime}$ então $u w \approx u^{\prime} w^{\prime}$.

As duas propriedades acima nos permite definir uma multiplicação em $F(X)$ da seguinte forma:

$$
[u] \odot[v]=[u v] .
$$

Claramente, $(F(X), \odot)$ é um grupo.

Vamos definir a seguinte função $i: X \rightarrow F(X)$ dada por $i(x)=[x], \forall x \in X$. Afirmamos que $F(X)$ é gerado por $i(X)$. De fato, se $\alpha \in F(X)$ então $\alpha=[w]$, para algum $w \in M(X \cup \bar{X})$. Mas, $w=x_{i_{1}}^{\xi_{1}} \ldots x_{i_{n}}^{\xi_{n}} . \operatorname{Logo}, \alpha=\left[x_{i_{1}}^{\xi_{1}} \ldots x_{i_{n}}^{\xi_{n}}\right]=\left[x_{i_{1}}^{\xi_{1}}\right] \ldots\left[x_{i_{n}}^{\xi_{n}}\right]=i\left(x_{1}\right)^{\xi_{1}} \ldots i\left(x_{n}\right)^{\xi_{n}}$.

Assim, cada elemento em $F(X)$ é escrito como combinação de elementos de $i(X)$.

Teorema 1.1.6. $(F(X), i)$ é livre sobre $X$.

Demonstração: Sejam $G$ um grupo e $f: X \rightarrow G$ uma função. Então $f$ se estende a uma função $\bar{f}: M(X \cup \bar{X}) \rightarrow G$ tal que $\bar{f}(x)=f(x), \forall x \in X$ e, para cada palavra $x_{i_{1}}^{\xi_{1}} \ldots x_{i_{n}}^{\xi_{n}}$, temos $\bar{f}\left(x_{i_{1}}^{\xi_{1}} \ldots x_{i_{n}}^{\xi_{n}}\right)=f\left(x_{i_{1}}\right)^{\xi_{1}} \ldots f\left(x_{i_{n}}\right)^{\xi_{n}}$.

Observemos que:

1. Se $w^{\prime}$ é obtida de $w$ através de uma redução elementar então $w$ e $w^{\prime}$ possuem a mesma imagem.

2. Se $w \approx w^{\prime}$ então $w$ e $w^{\prime}$ possuem a mesma imagem. 
Portanto, é possível definir $\varphi: F(X) \rightarrow G$ dada por $\varphi([w])=\bar{f}(w)$. Temos:

- $\varphi$ é homomorfismo;

- $\varphi \circ i(w)=\varphi(i(w))=\varphi([w])=\bar{f}(w)$;

- Suponhamos que exista $\psi: F(X) \rightarrow G$ outro homomorfismo tal que $f=\psi \circ i$. Como $i(X)$ gera $F(X), \varphi$ e $\psi$ coincidem nos geradores de $F(X)$. Portanto, $\varphi=\psi$.

Teorema 1.1.7. (Forma Normal para Grupos Livres): Existe exatamente uma palavra reduzida em cada classe de equivalência.

Observação 1.1.8. Geralmente vamos considerar $X$ como um subconjunto de $F(X)$ com $i$ sendo a função inclusão e, consequentemente, vamos omitir $i$ no futuro.

Frequentemente vamos identificar os elementos de $F(X)$ com as palavras reduzidas correspondentes. Algumas veses vamos precisar considerar palavras como elementos de $M(X \cup \bar{X})$ e outras vezes vamos considerá-las como elementos de $F(X)$.

Agora, vamos estabelecer algumas notações: escrevemos $w \equiv w^{\prime}$ quando $w$ e $w^{\prime}$ são a mesma palavra e $w=w^{\prime}$ se elas definirem o mesmo elemento em $F(X)$ ( ou seja, se elas são palavras equivalentes).

É fácil ver, sem usar o Teorema da Forma Normal, que se $w$ e $w^{\prime}$ são palavras reduzidas então existe uma única sequência de reduções elementares a qual podemos aplicar em $w w^{\prime}$ para obtermos uma palavra reduzida:

Exemplo 1.1.9. Vamos considerar $w=x y z^{-1} v x^{-1}$ e $w^{\prime}=x v^{-1} z t k$.

Temos, $w w^{\prime}=x y z^{-1} v x^{-1} x v^{-1} z t k=x y z^{-1} v v^{-1} z t k=x y z^{-1} z t k=x y t k$.

Observemos que a única sequência de reduções elementares é justamente a sequência feita acima. Note que não podemos omitir nenhum dos passos.

Proposição 1.1.10. $F(X)$ é isomorfo a $F(Y)$ se, e somente se, $|X|=|Y|$.

Definição 1.1.11. Um grupo $G$ é chamado grupo livre se $G$ for isomorfo a $F(X)$, para algum $X$. 
Se $i: F(X) \rightarrow G$ é um isomorfismo, então $i(X)$ é chamado base de $G$. Também dizemos que $G$ é livre em $i(X)$.

Desta definição, segue que:

Se $A$ e $B$ são bases de $G$ então $|A|=|B|$. De fato, pela hipótese temos $A=i(X)$ e $B=\bar{i}(Y)$ bases de $G$, com $i: F(X) \rightarrow G$, para algum $X$ e $\bar{i}: F(Y) \rightarrow G$, para algum $\mathrm{Y}$, isomorfismos. Assim, $\bar{i} \circ i: F(X) \rightarrow F(Y)$ é um isomorfismo. Pelo item anterior, $|X|=|Y|$. Portanto, $|A|=|B|$.

Definição 1.1.12. A cardinalidade da base de um grupo livre é chamado posto.

Proposição 1.1.13. Seja $X$ um subconjunto do grupo G. São equivalentes:

(i) G é livre com base $X$;

(ii) Cada elemento de $G$ pode ser escrito de maneira única como $g=x_{i_{1}}^{\xi_{1}} \ldots x_{i_{n}}^{\xi_{n}}$, para algum $n \geq 0, x_{i_{r}} \in X, \xi_{r}= \pm 1$ onde $\xi_{r+1} \neq-\xi_{r}$, se $i_{r+1}=i_{r}$;

(iii) $X$ gera $G$ e 1 não é igual a nenhum produto $x_{i_{1}}^{\xi_{1}} \ldots x_{i_{n}}^{\xi_{n}}$ com $n>0, x_{i_{r}} \in X, \xi_{r}= \pm 1$ onde $\xi_{r+1} \neq-\xi_{r}$, se $i_{r+1}=i_{r}$.

Demonstração: $(i i) \Rightarrow($ iii $)$ é óbvio.

$($ iii $) \Rightarrow(i i)$

Como $X$ gera $G$, para cada $g \in G, g=x_{i_{1}}^{\xi_{1}} \ldots x_{i_{n}}^{\xi_{n}}$, para algum $n \geq 0, x_{i_{r}} \in X, \xi_{r}= \pm 1$, onde $\xi_{r+1} \neq-\xi_{r}$ se $i_{r+1}=i_{r}$. E também, se existissem duas maneiras distintas de escrever $g$, para algum $g \in G$, então multiplicando um pelo inverso do outro, conseguiríamos escrever 1 como um produto não trivial de letras em $X$, contrariando a hipótese.

(iii) e $(i i) \Leftrightarrow(i)$

Se $G$ é livre em $X$ então $G$ possui as propriedades da hipótese uma vez que $F(X)$ também as possui. Se $G$ possui as propriedades $(i i)$ e $(i i i)$, vamos considerar o homomorfismo $\alpha: F(X) \rightarrow G$ induzido pela aplição identidade em $X$. Assim, como $G$ é gerado por $X$, segue que $\alpha$ é sobrejetor. E também, por (iii), $\alpha$ é injetor. Portanto, $\alpha$ é isomorfismo e, assim, $G$ é livre em $X$.

Seja $g$ um elemento de $F(X)$. Estamos considerando $g$ como uma palavra reduzida, que tem comprimento $|g|$. Se $h$ é outra palavra reduzida de $F(X)$, pode ser que $g h$ não seja 
uma palavra reduzida (mas este produto está em $F(X)$ e gh é uma classe de equivalência desta palavra). Quando o produto $g h$ for ainda uma palavra reduzida, dizemos que o produto gh é reduzido como escrito. Mais geralmente, se $g_{1}, \ldots, g_{k}$ são palavras reduzidas e $g_{1} \ldots g_{k}$ for uma palavra reduzida, dizemos que este produto é reduzido como escrito.

Temos as seguintes propriedades:

(i) $|g h| \leq|g|+|h|$ (a igualdade é válida se, e somente se, $g h$ for reduzida como escrito).

(ii) A primeira letra de $g h$ é a primeira letra de $g$ ou $g$ é cancelada completamente no produto $g h$. Isto acontece se, e somente se, $h \equiv g^{-1} k$, para alguma palavra reduzida $k$. Similarmente, $g h$ termina com a última letra de $h$ ou $h$ é cancelado completamente no produto $g h$. Isto acontece se, e somente se, $g \equiv k h^{-1}$, para alguma palavra reduzida $k$.

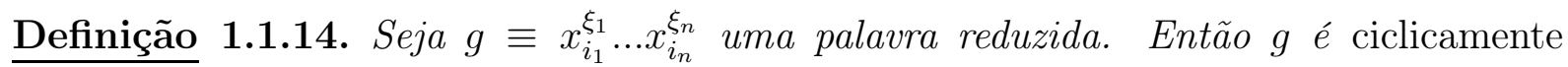
reduzida se ou $i_{n} \neq i_{1}$ ou $i_{n}=i_{1}$ com $\xi_{n} \neq-\xi_{1}$.

Claramente, 1 é ciclicamente reduzida.

Exemplo 1.1.15. 1. $g=x y z$ é ciclicamente reduzida.

2. $g^{\prime}=x y x$ é ciclicamente reduzida.

3. $g^{\prime \prime}=x y x^{-1}$ é não ciclicamente reduzida.

Temos ainda, mais algumas propriedades:

1. $g$ é ciclicamente reduzida se, e somente se, $g g$ é reduzida como escrito.

2. Se $g$ for ciclicamente reduzida então, indutivamente, $g^{n}$ será reduzida como escrito e $\left|g^{n}\right|=n|g|$.

3. Se $g \equiv u^{-1} v u$ for reduzida como escrito e $v$ for ciclicamente reduzida, então $g^{n}=$ $u^{-1} v^{n} u$ será reduzida como escrito.

Definição 1.1.16. Uma permutação cíclica da palavra $x_{i_{1}}^{\xi_{1}} \ldots x_{i_{n}}^{\xi_{n}}$ é qualquer palavra $x_{i_{r}}^{\xi_{r}} \ldots x_{i_{n}}^{\xi_{n}}$ $x_{i_{1}}^{\xi_{1}} \ldots x_{i_{r-1}}^{\xi_{r-1}}$. 
Exemplo 1.1.17. Seja $w=x y x^{-1} z y^{-1} x z^{-1}$. Então $z y^{-1} x z^{-1} x y x^{-1}$ é uma permutação cíclica de $w$.

Proposição 1.1.18. (i) Qualquer elemento de $F(X)$ é conjugado de uma palavra ciclicamente reduzida.

(ii) Qualquer permutação cíclica de uma palavra ciclicamente reduzida é ciclicamente reduzida.

(iii) Duas palavras reduzidas são conjugadas se, e somente se, elas são permutações cíclicas uma da outra.

Demonstração: $(i)$ Seja $g \equiv x_{i_{1}}^{\varepsilon_{1}} \ldots x_{i_{n}}^{\varepsilon_{n}}$ uma palavra reduzida mas não ciclicamente reduzida. Então $g \equiv x_{i_{1}}^{\varepsilon_{1}} g^{\prime} x_{i_{1}}^{-\varepsilon_{1}}$, onde $g^{\prime}$ é a palavra $x_{i_{2}}^{\varepsilon_{2}} \ldots x_{i_{n-1}}^{\varepsilon_{n-1}}$. O resultado segue por indução.

(ii) A palavra $x_{i_{n}}^{\varepsilon_{n}} x_{i_{1}}^{\varepsilon_{1}} \ldots x_{i_{n-1}}^{\varepsilon_{n-1}}$ é ciclicamente reduzida desde que, por hipótese, cada $i_{n} \neq i_{1}$ ou $\varepsilon_{n} \neq-\varepsilon_{1}$. O resultado segue indutivamente.

(iii) Qualquer permutação cíclica de uma palavra ciclicamente reduzida $g$ é um conjugado de $g$. Reciprocamente, tomemos qualquer conjugado $u g u^{-1}$ de $g$. Se $u^{-1} g u$ é reduzida como escrito, com $u \neq 1$, então $u^{-1} g u$ não é ciclicamente reduzido, desde que ela termina com a última letra de $u$ e começa com sua inversa, a primeira letra de $u^{-1}$. Se $u^{-1} g u$ não é reduzida como escrito então a primeira letra de $u$ é $x_{i_{1}}^{\varepsilon_{1}}$ ou $x_{i_{n}}^{-\varepsilon_{n}}$. Em ambos os casos, $u^{-1} g u=v^{-1} h v$, onde $|v|<|u|$ e $h$ é uma permutação cíclica de $g$. O resultado segue por indução no comprimento.

Proposição 1.1.19. Grupos livres são livres de torção, ou seja, se F for um grupo livre então:

$$
T(F)=\{w \in F ; o(w)<\infty\}=\{1\} .
$$

Demonstração: Sejam $F$ um grupo livre e $g \in F, g \neq 1$. Tomando o conjugado se necessário, podemos assumir que $g$ é ciclicamente reduzida.

Logo, $\left|g^{n}\right|=n|g| \neq 0$. Portanto, $g^{n} \neq 1$.

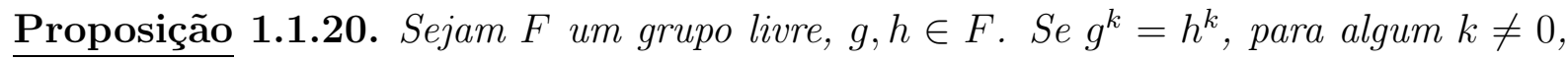
então $g=h$. 
Demonstração: Podemos assumir $k \geq 0$. Tomando o conjugado se necessário, podemos assumir $g$ ciclicamente reduzida. Portanto, $g^{k}$ é ciclicamente reduzida como escrito.

Se $h$ não é ciclicamente reduzida então $h^{k}$ não é ciclicamente reduzida como escrito. Logo, $h^{k} \neq g^{k}$, contrariando nossa hipótese. Logo, $h$ é ciclicamente reduzido e, portanto, $h^{k}$ é ciclicamente reduzido como escrito. Assim, se $g^{k}=h^{k}$ então $g \ldots g=h \ldots h$ e isto requer $h=g$.

Proposição 1.1.21. Sejam F um grupo livre e $g, h \in F$. Se $g h=h g$ então $<g, h>e ́$ cíclico; isto é, existe $u \in F$ e inteiros $r$ e s tais que $g=u^{r}$ e $h=u^{s}$.

\subsection{Geradores e relatores}

Proposição 1.2.1. Qualquer grupo $G$ é quociente de algum grupo livre.

Demonstração: Vamos definir a aplicação $I_{d}: G \rightarrow G, I_{d}(g)=g, \forall g \in G$. Esta aplicação pode ser estendida a um homomorfismo $\varphi: F(G) \rightarrow G$, definido por $\varphi(w)=$ $\varphi\left(g_{i_{1}}^{\xi_{1}} \ldots g_{i_{n}}^{\xi_{n}}\right)=I_{d}\left(g_{i_{1}}\right)^{\xi_{1}} \ldots I_{d}\left(g_{i_{n}}\right)^{\xi_{n}}=g_{i_{1}}^{\xi_{1}} \ldots g_{i_{n}}^{\xi_{n}}=w, \forall w=g_{i_{1}}^{\xi_{1}} \ldots g_{i_{n}}^{\xi_{n}} \in F(G)$. Claramente, $\varphi$ é sobrejetor.

Pelo Teorema do Homomorfismo, $\frac{F(G)}{\operatorname{ker} \varphi} \simeq G$.

Sejam $G$ um grupo, $X$ um conjunto e $\varphi: F(X) \rightarrow G$ um epimorfismo. $X$ é chamado um conjunto de símbolos geradores para $G$ (via $\varphi$ ) e a família $\{\varphi(x) ; x \in X\}$ é chamada família de geradores de $G$. Claramente, $G=<\varphi(X)>$. Desde que nós temos $\varphi(x)=\varphi(y)$ com $x \neq y$ nos referimos a família em vez de conjunto.

Vamos chamar ker $\varphi$ de conjunto de relatores de $G$ (via $\varphi$ ). Se $u=x_{i_{1}}^{\xi_{1}} \ldots x_{i_{n}}^{\xi_{n}}$ e $v=x_{j_{1}}^{\delta_{1}} \ldots x_{j_{n}}^{\delta_{n}}$ são palavras (não necessariamente reduzidas) com $u v^{-1}$ representando um elemento do $k \operatorname{er} \varphi$, e $\varphi\left(x_{i}\right)=a_{i}$, dizemos que $a_{i_{1}}^{\xi_{1}} \ldots a_{i_{n}}^{\xi_{n}}=a_{j_{1}}^{\delta_{1}} \ldots a_{j_{n}}^{\delta_{n}}$ é uma relação em $G$. Em particular, se $u$ representa um elemento em $\operatorname{ker} \varphi$ então a relação correspondente em $G$ é $a_{i_{1}}^{\xi_{1}} \ldots a_{i_{n}}^{\xi_{n}}=1$.

Para qualquer subconjunto $S$ de um grupo $H$, o fecho normal $<S>^{H}$ em $H$ do subgrupo $<S>$ é chamado o conjunto das consequências de $S$ em $H$, ou simplesmente subgrupo normal de $H$ gerado por $S$.

Se ker $\varphi$ é o conjunto das consequências de algum subconjunto $R$ de $F(X)$ chamamos $R$ o conjunto dos relatores definidores de $G$ (via $\varphi$ ). Temos um conjunto correspondente de relações definidoras. Também dizemos que a relação $u=v$ é uma consequência de 
um conjunto de relatores definidores (ou relações definidoras) se o relator correspondente $u v^{-1}$ é uma consequência dos relatores definidores.

Uma apresentação $<X ; R>^{\varphi}$ de $G$ consiste de um conjunto $X$, um epimorfismo $\varphi$ de $F(X)$ em $G$, e um conjunto $R$ de relatores definidores de $\mathrm{G}$ via $\varphi$. Frequentemente omitimos a menção de $\varphi$, especialmente quando $\varphi$ é a aplicação natural de $F(X)$ em $\frac{F(X)}{<R>F(X)}$ ou quando $\varphi$ é $1-1 \mathrm{em} X$ (neste caso consideramos $X$ como um subconjunto de $G$ ). Escreveremos $G=<X ; R>^{\varphi}$ quando $<X ; R>^{\varphi}$ for uma apresentação de $G$. As vezes, é mais conveniente substituir cada relator $r$ pela relação correspondente $r=1$, ou mais geralmente, por uma relação $u=v$, onde $r=u v^{-1}$ (ou $v^{-1} u$ ). Se for conveniente, podemos misturar relatores e relações. Por exemplo, se soubermos que um grupo $G$ gerado por um conjunto $X$ é abeliano, é mais fácil escrever relações do tipo $x y=y x, x, y \in X$, ao invés do relator correspondente $x^{-1} y^{-1} x y$, mesmo que não troquemos os outros relatores por relações.

Se ambos $X$ e $R$ forem finitos, vamos nos referir à apresentações finitas e à grupos finitamente apresentados.

Exemplo 1.2.2. 1. O grupo livre em $X$ tem uma apresentação $<X ; R>\operatorname{com} R=\emptyset$. De fato, vamos considerar $\psi: F(X) \rightarrow F(X)$ o homomorfismo bijetor induzido pela aplicação identidade $I: X \rightarrow X$. Desde que $\psi$ é injetora, segue que ker $\psi=\{1\}$. Assim, se $R=\emptyset$ então ker $\psi=<R>^{F(X)}=\{1\}$. Portanto, $F(X)=<X ; \emptyset>^{\psi}$.

2. A apresentação $<x, y ; x y^{2}=y^{3} x, y x^{2}=x^{3} y>$ representa o grupo trivial. De fato, basta notarmos que $x y^{4} x^{-1}=y^{6}, x^{2} y^{4} x^{-2}=y^{9}, y x^{2} y^{4} x^{-2} y^{-1}=y^{9}$ e $x^{3} y^{4} x^{-3}=$ $y x^{2} y^{4} x^{-2} x^{-1}$. Logo, $x^{2} y^{4} x^{-2}=x^{3} y^{4} x^{-3}, y^{4} x^{-2}=x y^{4} x^{-3}$ e $y^{4} x=x y^{4}$. Assim, $y^{6}=x y^{4} x^{-1}=y^{4} x x^{-1}=y^{4}$. Então, $y^{2}=1$. Logo, se $x y^{2}=y^{3} x$ entãa $y=1$. E também se $y x^{2}=x^{3} y$ então $x=1$. Portanto, $X=\{1\}$ e $F(X)=\{1\}$. Desde que $\varphi: F(X) \rightarrow G$ é um homomorfismo sobrejetor, temos $G=\{e\}$.

Teorema 1.2.3. (Teorema de Von Dick) Sejam $G=<X ; R>^{\varphi}, f: X \rightarrow H$ uma função em algum grupo $H$ e $\theta: F(X) \rightarrow H$ o homomorfismo correspondente. Então existe um homomorfismo $\psi: G \rightarrow H$ tal que $f(x)=\psi \circ \varphi(x), \forall x \in X$ se $\theta(r)=1, \forall r \in R$. Mais ainda, $\psi$ é um epimorfismo se $f(X)$ gera $H$.

Demonstração: Temos que $R \subseteq k e r \theta$, e, desde que ker $\varphi$ é o subgrupo normal gerado por $R$, temos $k e r \varphi \subseteq k e r \theta$. Segue, como sabemos, que o homomorfismo $\psi$ requerido pode 
ser definido se exigirmos que $\psi(g)=\theta(w)$ para qualquer $w$ tal que $\varphi(w)=g$. Claramente, $\varphi(G)=<f(X)>$, e então $\psi(G)=H$ se $f(X)$ gera $H$.

Observação 1.2.4. Como um caso particular deste teorema, a inclusão de $X$ em $X \cup Y$ induz um homomorfismo de $<X ; R>$ em $<X \cup Y ; R \cup S>$ para qualquer subconjunto $S$ de $F(X \cup Y)$. Este homomorfismo será usado no futuro sem explicitarmos como foi construído.

Pelo mesmo argumento usado no teorema, podemos demonstrar o resultado a seguir (ao qual também nos referiremos como Teorema de Von Dick): Sejam $R$ um subconjunto de um grupo $A$ e $\theta: A \rightarrow H$ um homomorfismo tal que $\theta(R)=\{1\}$. Então existe um homomorfismo $\psi: \frac{A}{<R>^{A}} \rightarrow H$ tal que $\theta=\psi \circ \pi$, onde $\pi$ é o epimorfismo canônico de $A$ em $\frac{A}{<R>^{A}}$.

Quando estivermos procurando por uma apresentação de um grupo $H$ o procedimento a seguir será muito útil: começamos determinando um conjunto $X$ de geradores de $H$. Então, encontraremos um conjunto $R$ de relatores de $H$ com geradores $X$ escolhido de modo que temos razão pra acreditar (ou pelo menos achar) que $R$ é um conjunto de relatores definidores. Pelo Teorema de Von Dick, existe um epimorfismo de $G=<X ; R>$ em $H$.

Pode ser que os relatores em $R$ nos possibilitem escrever os elementos de $G$ numa forma simples que nos permita enxergar que a aplicação é injetora. Alternativamente, se $H$ for finito, pode ser que possamos mostrar que $G$ é finito e que $|G| \leq|H|$, o que novamente mostra que a aplicação é injetora.

Exemplo 1.2.5. O grupo cíclico $\mathbb{Z}_{n}$ de ordem $n$ tem apresentação $<x ; x^{n}>$ desde que o grupo com esta apresentação certamente tem um epimorfismo em $\mathbb{Z}_{n}$ e é fácil ver que ele tem no máximo n elementos.

Claramente, um grupo $G$ pode ter várias apresentações, mesmo para dados $X$ e $\varphi$. Vamos agora olhar para apresentações diferentes de um mesmo grupo e compará-las.

Seja $<X ; R>^{\varphi}$ uma apresentação de um grupo $G$. Então $<X ; R \cup S>^{\varphi}$, para qualquer $S \subset<R>^{F(X)}$ também é uma apresentação para $G$. Dizemos que $<X ; R \cup S>^{\varphi}$ vem de $<X ; R>^{\varphi}$ por uma Transformação Geral de Tietze de Tipo I e $<X ; R>^{\varphi}$ vem de $<X ; R \cup S>^{\varphi}$ por uma Transformação de Geral Tietze de Tipo I'. Se $|S|=1$, vamos nos referir a uma Transformação Simples de Tietze. 
Sejam $Y$ um conjunto tal que $X \cap Y=\emptyset$ e $u_{y}$ um elemento de $F(X)$ para cada $y \in Y$. Então $<X \cup Y ; R \cup\left\{y u_{y}^{-1}, \forall y \in Y\right\}>^{\psi}$ também é uma apresentação de $G$, onde $\psi(x)=\varphi(x)$ e $\psi(y)=\varphi\left(u_{y}\right)$. De fato, seja $N$ o subgrupo normal de $F(X \cup Y)$ gerado por $R \cup\left\{y u_{y}\right\}^{-1}$. Então pelo Teorema do Homomorfismo, $\psi$ induz um epimorfismo $\pi: \frac{F(X \cup Y)}{N} \rightarrow G$, desde que $\psi\left(R \cup\left\{y u_{y}{ }^{-1}\right\}\right)=\{1\}$. Mas, pelo Teorema de Von Dick, existe também um epimorfismo $\theta: G \rightarrow \frac{F(X \cup Y)}{N} \operatorname{com} \theta(\varphi(x))=x N$. Claramente, $\pi \circ \theta$ é a aplicação identidade. E também, desde que $\theta \circ \pi(y N)=\theta \circ \psi(y)=\theta \circ \varphi\left(u_{y}\right)=u_{y} N=y N$, temos $\theta \circ \pi$ a aplicação identidade.

Dizemos que $<X \cup Y ; R \cup\left\{y u_{y}^{-1}, \forall y \in Y\right\}>^{\psi}$ vem de $<X ; R>^{\varphi}$ por uma Transformação Geral de Tietze de tipo II e $<X ; R>^{\varphi}$ vem de $<X \cup Y ; R \cup\left\{y u_{y}^{-1}, \forall y \in Y\right\}>^{\psi}$ por uma Transformação Geral de Tietze de Tipo $I I^{\prime}$. Se $|Y|=1$, nos referiremos a uma Transformação de Tietze Simples.

Claramente, uma Transformação Geral de Tietze com $|S|$ ou $|Y|$ finitos pode ser obtida através de um número finito de Transformações de Tietze Simples.

Teorema 1.2.6. Qualquer duas apresentações de um mesmo grupo podem ser obtidas uma da outra por uma sequência de Transformações gerais de Tietze. Se ambas as apresentações são finitas então cada uma pode ser obtida da outra por uma sequência de Transformações de Tietze Simples.

\subsection{Produtos livres}

Definição 1.3.1. Sejam $\left\{G_{\alpha}\right\}_{\alpha \in \Gamma}$ uma família de grupos, $G$ um grupo e $i_{\alpha}: G_{\alpha} \rightarrow G$ homomorfismo, para todo $\alpha \in \Gamma$. Dizemos que o par $\left(G,\left\{i_{\alpha}\right\}\right)$ é um produto livre dos grupos $G_{\alpha}$ se, para cada grupo $H$, para cada homomorfismo $f_{\alpha}: G_{\alpha} \rightarrow H$, existir um único homomorfismo $f: G \rightarrow H$ tal que $f_{\alpha}=f \circ i_{\alpha}$, para todo $\alpha \in \Gamma$.

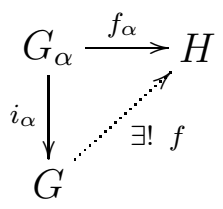

Proposição 1.3.2. Se $\left(G,\left\{i_{\alpha}\right\}\right)$ e $\left(H,\left\{j_{\alpha}\right\}\right)$ são produtos livres de uma família $G_{\alpha}$ de grupos então existe um único isomorfismo $f: G \rightarrow H$ tal que $f \circ i_{\alpha}=j_{\alpha}$, para todo 
$\alpha \in \Gamma$.

Demonstração: Pela hipótese, existem homomorfismos $f: G \rightarrow H$ e $f^{\prime}: H \rightarrow G$ tais que $f \circ i_{\alpha}=j_{\alpha}$ e $f^{\prime} \circ j_{\alpha}=i_{\alpha}$, para todo $\alpha \in \Gamma$. Assim, $f^{\prime} \circ f \circ i_{\alpha}=i_{\alpha}$. A unicidade dos homomorfismos na definição fornece $f^{\prime} \circ f=I d_{G}$ e $f \circ f^{\prime}=I d_{H}$. Portanto, $f^{\prime}=f^{-1}$.

Teorema 1.3.3. Qualquer familia $\left\{G_{\alpha}\right\}_{\alpha \in \Gamma}$ de grupos admite um produto livre.

Observação 1.3.4. O produto livre de uma família de grupos $\left\{G_{\alpha}\right\}$ será denotado por $* G_{\alpha}$ enquanto o produto livre de dois grupos é geralmente denotado por $G_{1} * G_{2}$. Mais geralmente, o produto livre de $n$ grupos $G_{i}$ é denotado por $G_{1} * \ldots * G_{n}$.

Exemplo 1.3.5. O grupo livre $F(X)$ é o produto livre de grupos $C_{x}, \forall x \in X$ onde $C_{x} e ́$ o grupo cíclico infinito gerado por $x$.

Teorema 1.3.6. (Forma Normal) Seja $\left(G,\left\{i_{\alpha}\right\}\right)$ o produto livre da família de grupos $\left\{G_{\alpha}\right\}$. Então:

(i) Cada $i_{\alpha}$ é um monomorfismo.

(ii) Considerando $i_{\alpha}$ como a inclusão, qualquer elemento de $G$ pode ser escrito de maneira única como $g_{1} \ldots g_{n}$, para algum $n \geq 0, g_{i} \in G_{\alpha_{i}}$ para algum $\alpha_{i}, g_{i} \neq 1$ e $\alpha_{r} \neq \alpha_{r+1}$, para $r<n$.

Proposição 1.3.7. Sejam $\left\{G_{\alpha}\right\}$ subgrupos de um grupo G. Então são equivalentes:

(i) $G$ é o produto livre dos subgrupos $G_{\alpha}$;

(ii) Todo elemento de $G$ pode ser escrito de maneira única como $g_{1} \ldots g_{n}$, com $n \geq 0$, $g_{i} \in G_{\alpha_{i}}, g_{i} \neq 1$ e $\alpha_{i} \neq \alpha_{i+1}$

(iii) $G$ é gerado pelos subgrupos $G_{\alpha}$ e 1 não pode ser escrito como um produto $g_{1} \ldots g_{n}$, com $n>0, g_{i} \in G_{\alpha_{i}}, g_{i} \neq 1$ e $\alpha_{i} \neq \alpha_{i+1}$.

\subsection{Push-outs e produtos livres amalgamados}

Definição 1.4.1. Sejam $G_{0}, G_{1}, G_{2}$ grupos e $i_{1}: G_{0} \rightarrow G_{1}$ e $i_{2}: G_{0} \rightarrow G_{2}$ homomorfismos. Sejam $G$ um grupo, $j_{1}: G_{1} \rightarrow G, j_{2}: G_{2} \rightarrow G$ homomorfismos. Dizemos que $\left(G, j_{1}, j_{2}\right)$ é push-out de $\left(i_{1}, i_{2}\right)$ se: 
(i) $j_{1} \circ i_{1}=j_{2} \circ i_{2}$;

(ii) Para cada grupo $H$ e homomorfismos $\varphi_{r}: G_{r} \rightarrow H, r=1,2 \operatorname{com} \varphi_{1} \circ i_{1}=\varphi_{2} \circ i_{2}$ existir um único homomorfismo $\varphi: G \rightarrow H$ tal que $\varphi_{r}=\varphi \circ j_{r}, r=1,2$

Assim, dizemos que temos um quadrado push-out:

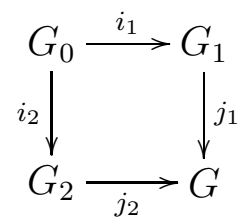

Observe que o push-out é único a menos de isomorfismos.

Teorema 1.4.2. Qualquer par $\left(i_{1}, i_{2}\right)$ admite um push-out.

Exemplo 1.4.3. Se $G_{2}$ é o grupo trivial então o push-out é $G=\frac{G_{1}}{\left.<i_{1}\left(G_{0}\right)\right\rangle^{G_{1}}}$.

Os homomorfismos $j_{1}$ e $j_{2}$ não precisam ser injetores, mesmo se ou $i_{1}$ ou $i_{2}$ for injetor. Por exemplo, suponha que $G_{1}$ seja simples, $i_{1}$ seja injetor e $i_{2}$ sobrejetor mas não injetor.

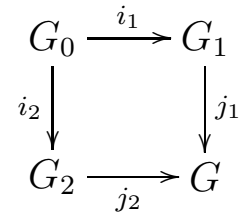

Vamos tomar $w \in G_{0} \operatorname{com} w \neq 1$ e $i_{2}(w)=1$. Como $i_{1}$ é injetor, ker $i_{1}=\{1\}$. Logo, como $w \neq 1$ temos $i_{1}(w) \neq 1$. E mais, $j_{1} \circ i_{1}(w)=j_{2} \circ i_{2}(w)=j_{2}(1)=1$. Como $G_{1}$ é simples, então $G_{1}=<i_{1}\left(G_{0}\right)>^{G_{1}}$ e como $j_{1} \circ i_{1}(w)=1, \forall w \in G_{0}, w \neq 1$, segue que $j_{1}$ é trivial. Como $i_{2}$ é sobrejetor, $i_{2}\left(G_{0}\right)=G_{2}$ e como $j_{2} \circ i_{2}=j_{1} \circ i_{1}=1, \forall w \in G_{0}$, temos que $j_{2}$ é trivial. Portanto, $G=\{1\}$.

Quando $i_{1}$ e $i_{2}$ forem injetores, chamamos o push-out $G$ de produto livre amalgamado de $G_{1}$ e $G_{2}$ com $G_{0}$ amalgamado. Neste caso, geralmente consideramos $G_{0}$ como um subgrupo de $G_{1}$ e $G_{2}$ e $i_{1}, i_{2}$ como inclusões. A notação usual para esta situação é

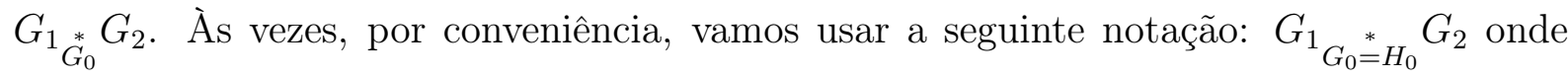
$G_{0} \subseteq G_{1}, H_{0} \subseteq G_{2}, i_{1}$ a inclusão e $i_{2}$ isomorfismo entre $G_{0}$ e $H_{0}$. Para maior precisão, pode-se mencionar o isomorfismo específico de $G_{0}$ para $H_{0}$. 


\subsection{Apêndice}

Nesta seção faremos um estudo breve e informal sobre o problema da palavra nos grupos finitamente apresentados. Veremos um algoritmo em especial, a saber, Algoritmo de Dehn para resolvermos o problema em questão. Este algoritmo será usado no artigo que estudaremos nessa dissertação quando estivermos abordando o problema da palavra nos grupos de tranças em superfícies tanto no caso orientável quanto no caso não orientável. Mais detalhes podem ser encontrados em [LH] e [LS].

\subsubsection{O problema da palavra}

Por um grupo definido por seus geradores e relações, o problema da palavra consiste basicamente, em encontrar um algoritmo para decidir quando uma dada palavra representa o elemento identidade.

A fim de estudar o Algoritmo de Dehn precisamos de duas definições que virão a seguir:

Definição 1.5.1. Seja $F$ um grupo livre sobre um conjunto $X$. Um subconjunto $R$ de $F$ é dito simetrizado se todos seus elementos são ciclicamente reduzidos e, para cada $r \in R$, todos os conjugados ciclicamente reduzidos de $r$ e $r^{-1}$ também pertencem a $R$.

Definição 1.5.2. Um conjunto $R$ é dito recursivo se existe um algoritmo para decidir quando um dado $r$ pertence a $R$.

\subsubsection{O algoritmo de Dehn}

Vamos considerar um grupo $G$ com apresentação $<x_{1}, \ldots, x_{n} ; R>$ onde $R$ é um conjunto recursivo simetrizado de relatores definidores e que esteja verificado que palavras não triviais livremente reduzidas que são 1 em $G$ contém mais que a metade de algum elemento de $R$. Seja $w$ uma palavra não trivial de G. Se $w=1$ em $G$, então $w$ possui alguma fatoração $w \equiv b c d$, onde para algum $r \in R, r \equiv c t$, com $|t|<|c|$. Agora, esse tal $r$ satisfaz $|r|<2|w|$. O conjunto $S$ das palavras em $\left\{x_{1}, \ldots, x_{n}\right\}$ que têm comprimento menor que $2|w|$ é finito. Desde que $R$ é recursivo podemos efetivamente listar todos os elementos de $R^{\prime}=R \cap S$. Se encontrarmos um $r$ adequado então $w=b t^{-1} d$ em $G$, e 
$b t^{-1} d$ é uma palavra de comprimento menor. Um número finito de passos ou nos dão 1 como resultado, dando uma prova que $w=1 \mathrm{em} G$, ou nos dão uma palavra $w^{*}$ a qual não pode ser "simplificada", estabelecendo $w \neq 1 \mathrm{em} G$.

Exemplo 1.5.3. Vamos considerar $G=<a, b ; a b a^{-1} b^{-1}>$. Então a apresentação de $G$ onde o conjunto de relatores é recursivo simetrizado é dada por

$$
G=<a, b ; a b a^{-1} b^{-1}, b^{-1} a b a^{-1}, b a^{-1} b^{-1} a, a b^{-1} a^{-1} b, a^{-1} b a b^{-1}, a^{-1} b^{-1} a b, b^{-1} a^{-1} b a>.
$$

Seja $w=a b a^{-1} b^{-1} a b a^{-1} b^{-1}$. Vamos aplicar o algoritmo de Dehn para mostrar que $w=1$ em $G$ :

- $w$ tem a fatoração bcd onde $b=a b a^{-1} b^{-1}, c=a b a^{-1} b^{-1}$ e d =

- para $r=a b a^{-1} b^{-1}$, temos $r \equiv c t$, com $t=1, \operatorname{com}|r|=4<16$;

Então, temos $w=b t^{-1} d=a b a^{-1} b^{-1}$, uma palavra de comprimento menor. Aplicando novamente o passo anterior, temos $w=1 \mathrm{em} G$.

Observação 1.5.4. A hipótese de que $R$ é um conjunto simetrizado não pode ser descartada. De fato, vamos considerar o mesmo grupo do exemplo anterior e suponhamos $R$ não seja simetrizado. Assim, o único elemento $r$ de $R$ será $a b a^{-1} b^{-1}$. Vamos tomar $w=$ $a^{-1} b^{-1} a b$. Assim, pelo Algoritmo de Dehn w seria diferente de 1 já que não existiria $r \in R$ que satisfizesse as condições do algoritmo. Por outro lado, $w=a^{-1} b^{-1} a b=a^{-1} b^{-1} b a=1$ já que $a b=b a$. Portanto, uma contradição.

Mas, se considerarmos $R$ um conjunto simetrizado, o algoritmo fica bem definido, e, escolhendo $r=b a^{-1} b^{-1} a \in R, w=1$ pelo Algoritmo de Dehn. 


\section{Capítulo}

2

\section{Apresentações de produtos diretos,}

\section{semidiretos e extensões de grupos}

\subsection{Apresentação de produtos diretos}

Proposição 2.1.1. Se $G, H$ são grupos apresentados por $<X ; R>e<Y$; $S>$ respectivamente, então seu produto direto $G \times H$ tem a apresentação $<X, Y ; R, S,[X, Y]>$, onde $[X, Y]$ denota o conjunto dos comutadores $\left\{x^{-1} y^{-1} x y ; x \in X, y \in Y\right\}$.

Demonstração: Vamos denotar por $D$ o grupo que possui a seguinte apresentação: $<X, Y ; R, S,[X, Y]>$. Mostremos que $D$ é isomorfo a $G \times H$.

Vamos considerar as inclusões $i_{X}: X \rightarrow D$ e $i_{Y}: Y \rightarrow D$. Pelo Teorema de Von Dick, estas inclusões induzem homomorfismos $\bar{i}_{X}: G \rightarrow D$ e $\bar{i}_{Y}: H \rightarrow D$ respectivamente.

Os relatores $[X, Y]$ garantem que os elementos da imagem de $\bar{i}_{X}$ comutam com os elementos da imagem de $\bar{i}_{Y}$ em $D$. Temos então o homomorfismo $\alpha: G \times H \rightarrow D$ dado por $\alpha(g, h)=\bar{i}_{X}(g) \bar{i}_{Y}(h)$. Resta mostrarmos agora que $\alpha$ é isomorfismo. Primeiramente, vamos observar que $\alpha(x, 1)=x$, para cada $x \in X$ e $\alpha(1, y)=y$, para cada $y \in Y$.

Agora, vamos considerar a aplicação $\gamma: X \cup Y \rightarrow G \times H$ dada por $\gamma(x)=(x, 1)$ e $\gamma(y)=(1, y)$. Pelo Teorema de Von Dick, existe um homomorfismo $\beta: D \rightarrow G \times H$ que estende $\gamma$. Temos que $\beta$ nada mais é que o inverso de $\alpha$ pois $\beta \circ \alpha$ e $\alpha \circ \beta$ fixam os geradores de $D$ e de $G \times H$ respectivamente. Portanto $\alpha$ é isomorfismo, como queríamos. 


\subsection{Produtos semidiretos}

Para a construção da apresentação para os produtos semidiretos vamos precisar de dois grupos $G$ e $A$ e um homomorfismo $\alpha: G \rightarrow$ AutA. A aplicação $\alpha$ determina uma ação de $G$ em A, dada por:

$$
a^{x}=\alpha(x)(a), a \in A, x \in G
$$

Observemos que $\alpha(x)$ é um automorfismo de A.

Desde que $\alpha$ é um homomorfismo, seguem algumas propriedades: para cada $a, b \in A$, $x, y \in G$

1. $(a b)^{x}=a^{x} b^{x}$

2. $a^{x y}=\left(a^{x}\right)^{y}$

3. $a^{1}=a$.

Agora, vamos considerar o produto cartesiano $K=G \times A$, com a operação $(x, a)(y, b)=$ $\left(x y, a^{y} b\right)$. As propriedades acima nos garantem que os axiomas de grupo valem para esta operação, onde o elemento neutro é dado por $(1,1)$ e o inverso de $(x, a)$ é dado por $\left(x^{-1},\left(a^{-1}\right)^{x^{-1}}\right)$.

Definição 2.2.1. O grupo $K$ definido acima é chamado produto semidireto de $\mathrm{G}$ em A (com respeito a $\alpha$ ) e será denotada por $A] G$.

Claramente, as aplicações $A \rightarrow K$ e $G \rightarrow K$ dadas por $a \longmapsto(e, a)$ e $x \longmapsto(x, e)$ respectivamente são homomorfismos e é de costume identificarmos $A$ e $G$ com suas imagens em $K$. Então $A$ é um subgrupo normal em $K$, com complemento $G$, isto é, $G$ é um subgrupo de $K, A \cap G=E$, onde $E=\{1\}, G A=K$.

A razão para o nome produto semidireto é clara agora: no caso especial em que a $\operatorname{Im} \alpha=E$, a ação definida é trivial e a operação definida será o produto direto $G \times A$.

Não é difícil escrevermos uma apresentação para $A] G$ em termos das apresentações para $A$ e $G$ e da aplicação $\alpha$. Esta apresentação será estudada numa situação mais geral no próximo parágrafo. 


\subsection{Apresentação de extensões de grupos}

\subsubsection{Conceitos básicos}

Definição 2.3.1. Sejam $G, \tilde{G}$, A grupos. Dizemos que $\tilde{G}$ é uma extensão do grupo $G$ por $A$ se existir um subgrupo normal $N$ de $\tilde{G}$ tal que $A$ é isomorfo a $N$ e o quociente $\frac{\tilde{G}}{N} e ́$ isomorfo a $G$, ou seja, $A \stackrel{\alpha}{\cong} N$ e $\frac{\tilde{G}}{N} \stackrel{\beta}{\simeq} G$, onde $\alpha$ e $\beta$ são os respectivos isomorfismos.

Vamos ver agora alguns casos em que surgem extensões de grupos:

1. Seja $l: A \rightarrow \tilde{G}$ um mergulho normal. Então, $I m l \triangleleft \tilde{G}$. Assim, vamos definir $N=I m l$ e $G=\frac{\tilde{G}}{I m l}$. Logo, temos $A \simeq I m l$ pela própria definição de mergulho normal e, pelo modo que tomamos $G$, temos $\frac{\tilde{G}}{\operatorname{Iml}} \simeq G$.

2. Seja $\vartheta: \tilde{G} \rightarrow G$ um epimorfismo. Defina $A=k e r \vartheta$. Temos, ker $\triangleleft \tilde{G}$. Pelo Teorema do Homomorfismo, $\frac{\tilde{G}}{k e r \vartheta} \simeq G$. Portanto $\tilde{G}$ é uma extensão de $G$ por $A$.

Para os nossos propósitos, o melhor jeito de ilustrarmos a extensão de grupos é a seguinte:

$$
A \stackrel{\alpha}{\longrightarrow} N \stackrel{i}{\longrightarrow} \tilde{G} \quad \tilde{G} \stackrel{p}{\longrightarrow} \frac{\tilde{G}}{N} \stackrel{\beta}{\longrightarrow} G
$$

onde $i$ é a aplicação inclusão e $p$ é a aplicação natural.

A partir dos casos mencionados acima, obtemos o seguinte diagrama:

$$
A \stackrel{l}{\longrightarrow} \tilde{G} \stackrel{\vartheta}{\longrightarrow} G
$$

onde:

1. $l$ é um homomorfismo injetor, ou seja, $\operatorname{kerl}=\{1\}=E$;

2. $\vartheta$ é um homorfismo sobrejetor, ou seja, $\operatorname{Im\vartheta }=G$;

3. $I m l=k e r \vartheta$. De fato, seja $y \in I m l$. Logo, existe $a \in A$ tal que $y=l(a)$, ou seja, existe $a \in A$ tal que $y=l(a)=i \circ \alpha(a)=\alpha(a) \in N$. Portanto $\operatorname{Iml} \subseteq N$. Reciprocamente, se $y \in N$ então, desde que $\alpha$ é isomorfismo, existe $a \in A$ tal que $y=\alpha(a)=i \circ \alpha(a)=l(a)$. Logo, existe $a \in A$ tal que $y=l(a)$, ou seja, $y \in I m l$. Assim, $N \subseteq I m l$. Portanto, Iml $=N$. 
Por outro lado, se $w \in k e r \vartheta$ então $w \in \tilde{G}$ e $\vartheta(w)=1$. Logo, $\beta \circ p(w)=1$. Desde que $\beta$ é ismorfismo, temos $p(w)=1$. Logo, $w \in N$. Assim, $k e r \vartheta \subseteq N$. De forma análoga, temos $N \subseteq k e r \vartheta$. Assim, $N=k e r \vartheta$

Portanto, $I m l=k e r \vartheta$.

Definição 2.3.2. Uma sequência:

$$
A_{0} \stackrel{\alpha_{0}}{\longrightarrow} A_{1} \stackrel{\alpha_{1}}{\longrightarrow} \cdots \longrightarrow A_{n-1} \stackrel{\alpha_{n-1}}{\longrightarrow} A_{n}
$$

onde os $A_{i}, i=0, \ldots, n$ são grupos e os $\alpha_{i}, i=0, \ldots, n$ são homomorfismos é chamada sequência exata se $\operatorname{Im}_{\alpha_{i}-1}=k e r \alpha_{i}, \operatorname{com} 1 \leq i \leq n-1$.

Em particular, se $A_{0}=A_{n}$ são grupos triviais e $n=4$, dizemos que a sequência é exata curta. Neste caso, temos o seguinte diagrama:

$$
1 \longrightarrow A_{1} \stackrel{\alpha_{1}}{\longrightarrow} A_{2} \stackrel{\alpha_{2}}{\longrightarrow} A_{3} \longrightarrow 1
$$

onde $\alpha_{0}$ e $\alpha_{3}$ são triviais e as condições de exatidão de $A_{1}, A_{2}$ e $A_{3}$ respectivamente são $\operatorname{ker} \alpha_{1}=\{1\}, I m \alpha_{1}=\operatorname{ker} \alpha_{2}$ e Im $\alpha_{2}=A_{3}$ que são as mesmas condições que as propriedades 1, 2 e 3 enunciadas anteriormente possuem.

Assim, podemos pensar na extensão de grupos como uma sequência exata curta:

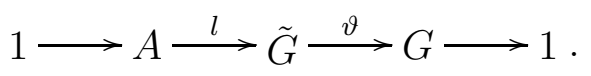

Definição 2.3.3. Um diagrama é um grafo dirigido cujos vértices são grupos e as arestas são homomorfismos entre seus pontos extremos. Um tal diagrama é comutativo se, dados quaisquer dois vértices e qualquer caminho entre eles, a composição dos homomorfismos correspondentes são iguais.

Lema 2.3.4. (Lema Dos Cinco) Consideremos o seguinte diagrama comutativo cujas 
linhas são sequências exatas:

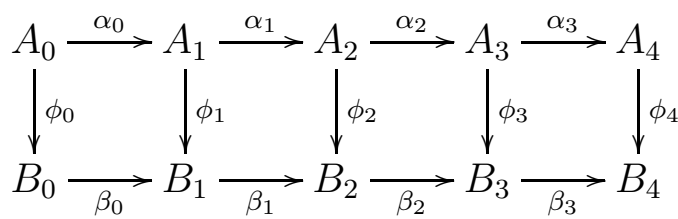

Se $\phi_{0}, \phi_{1}, \phi_{3}$ e $\phi_{4}$ são isomorfismos então $\phi_{2}$ também o é.

Demonstração: Temos de imediato que $\phi_{2}$ é um homomorfismo. Estão basta mostrarmos que $\phi_{2}$ é bijeção. Afirmamos que $\phi_{2}$ é injetor. De fato, seja $a \in k e r \phi_{2}$. Logo, $\phi_{2}(a)=1$ e assim $\beta_{2} \circ \phi_{2}(a)=1$. Pela comutatividade no quadrado 3 pela direita, temos $\beta_{2} \circ \phi_{2}=\phi_{3} \circ \alpha_{2}$. Logo, $\phi_{3}\left(\alpha_{2}(a)\right)=1$, ou seja, $\alpha_{2}(a) \in \operatorname{ker} \phi_{3}$. Desde que $\phi_{3}$ é injetor, segue que $\alpha_{2}(a)=1$, ou seja, $a \in k e r \alpha_{2}$. Pela exatidão em $A_{2}$, temos $\operatorname{Im} \alpha_{1}=k e r \alpha_{2}$. Logo, $a \in I m \alpha_{1}$ e assim, existe $a_{1} \in A_{1}$ tal que $\alpha_{1}\left(a_{1}\right)=a$.

Por outro lado, pela comutatividade do quadrado 2 pela direita, temos $\beta_{1} \circ \phi_{1}=\phi_{2} \circ \alpha_{1}$. Logo, $\beta_{1}\left(\phi_{1}\left(a_{1}\right)\right)=\phi_{2}(a)=1$ e assim, $\phi_{1}\left(a_{1}\right) \in \operatorname{ker} \beta_{1}$. Pela exatidão em $B_{1}$, temos $\operatorname{Im} \beta_{0}=\operatorname{ker} \beta_{1}$. Logo, $\phi_{1}\left(a_{1}\right) \in \operatorname{Im} \beta_{0}$. Logo, existe $b_{0} \in B_{0}$ tal que $\beta_{0}\left(b_{0}\right)=\phi_{1}\left(a_{1}\right)$. Como $\phi_{0}$ é sobrejetor, existe $a_{0} \in A_{0}$ tal que $\phi_{0}\left(a_{0}\right)=\phi_{1}\left(a_{1}\right)$. Pela comutatividade no quadrado 1 , segue que $\beta_{0} \circ \phi_{0}=\phi_{1} \circ \alpha_{0}$. Logo, $\beta_{0} \circ \phi_{0}\left(a_{0}\right)=\phi_{1} \circ \alpha_{0}\left(a_{0}\right)$ e então $\phi_{1}\left(a_{1}\right)=\phi_{1}\left(\alpha_{0}\left(a_{0}\right)\right)$. Desde que $\phi_{1}$ é injetor, segue que $\alpha_{0}\left(a_{0}\right)=a_{1}$, ou seja, $a_{1} \in \operatorname{Im} \alpha_{0}$ e, pela exatidão de $A_{1}$, temos $\operatorname{Im} \alpha_{0}=k e r \alpha_{1}$. Logo, $a_{1} \in k e r \alpha_{1}$, ou seja, $\alpha_{1}\left(a_{1}\right)=1$. Mas provamos acima que $\alpha_{1}\left(a_{1}\right)=a$. Portanto, $a=1$ como queríamos.

Agora, afirmamos que $\phi_{2}$ é sobrejetor. De fato, seja $b \in B_{2}$. Logo, pela definição, $\beta_{2}(b) \in B_{3}$. Como $\phi_{3}$ é sobrejetor, existe $a_{3} \in A_{3}$ tal que $\phi_{3}\left(a_{3}\right)=\beta_{2}(b)$. Assim, $\phi_{3}\left(a_{3}\right) \in \operatorname{Im} \beta_{2}=\operatorname{ker} \beta_{3}$ pela exatidão em $B_{3}$.

Portanto, $\beta_{3} \circ \phi_{3}\left(a_{3}\right)=1$. Logo, pela comutatividade do diagrama no quadrado 4 , segue que $\phi_{4} \circ \alpha_{3}\left(a_{3}\right)=1$. Como $\phi_{4}$ é injetor, segue que $\alpha_{3}\left(a_{3}\right)=1$, e assim, pela exatidão em $A_{3}$, temos $a_{3} \in \operatorname{ker} \alpha_{3}=I m \alpha_{2}$. Assim, existe $a_{2} \in A_{2}$ tal que $\alpha_{2}\left(a_{2}\right)=a_{3}$. Pela comutatividade do quadrado 3 , temos $\beta_{2} \circ \phi_{2}=\phi_{3} \circ \alpha_{2}$. Logo, $\beta_{2}\left(\phi_{2}\left(a_{2}\right)\right)=\phi_{3}\left(a_{3}\right)=\beta_{2}(b)$.

Seja $\left(\beta_{2}\left(\phi_{2}\left(a_{2}\right)\right)\right)^{-1}$ o inverso de $\beta_{2}\left(\phi_{2}\left(a_{2}\right)\right)$. Logo, temos $1=\beta_{2}(b)\left(\beta_{2}\left(\phi_{2}\left(a_{2}\right)^{-1}\right)=\right.$ $\beta_{2}\left(b \phi_{2}\left(a_{2}\right)^{-1}\right)$. Assim, pela exatidão em $B_{2}$, temos $b \phi_{2}\left(a_{2}\right)^{-1} \in \operatorname{ker} \beta_{2}=\operatorname{Im} \beta_{1}$. Logo, $b \phi_{2}\left(a_{2}\right)^{-1} \in \operatorname{Im} \beta_{1}$, ou seja, existe $b_{0} \in B_{0}$ tal que $\beta_{0}\left(b_{0}\right)=\phi_{1}\left(a_{1}\right)$. Como $b_{1} \in B_{1}$ e $\phi_{1}$ é sobrejetor, existe $a_{1} \in A_{1}$ tal que $\phi_{1}\left(a_{1}\right)=b_{1}$. Assim, $\beta_{1}\left(\phi_{1}\left(a_{1}\right)\right)=b \phi_{2}\left(a_{2}\right)^{-1}$. Por outro lado, pela comutatividade do quadrado 2 , temos $\phi_{2}\left(\alpha_{1}\left(a_{1}\right)\right)=b \phi_{2}\left(a_{2}\right)^{-1}$. Logo, $\phi_{2}\left(\alpha_{1}\left(a_{1}\right)\right) \phi_{2}\left(a_{2}\right)=b$ e, como $\phi_{2}$ é homomorfismo, temos $\phi_{2}\left(\alpha\left(a_{1}\right) a_{2}\right)=b, \operatorname{com} \alpha_{1}\left(a_{1}\right) a_{2} \in$ 
$A_{2}$. Portanto, $\phi_{2}$ é sobrejetor.

\subsubsection{O teorema principal}

Suponhamos que são dadas uma extensão:

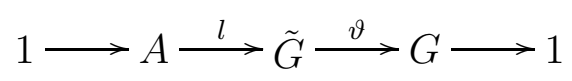

e apresentações $G=<X ; R>, A=<Y ; S>$ para $G$ e $A$. Nosso objetivo é conseguir uma apresentação para $\tilde{G}$.

Primeiro, seja $\tilde{Y}=\{\tilde{y}=l(y) ; y \in Y\}$ e seja $\tilde{S}=\{\tilde{s} ; s \in S\}$ o conjunto das palavras em $\tilde{Y}$ obtidas de $S$ substituindo cada $y$ por $\tilde{y}$ sempre que $y$ aparecer. Seja $\tilde{X}=\{\tilde{x} ; x \in X\}$ os membros de uma transversal para $\operatorname{Iml}$ em $\tilde{G}$ tal que $\vartheta(\tilde{x})=x$, para todo $x \in X$. Além disso, para cada $r \in R$, seja $\tilde{r}$ a palavra em $\tilde{X}$ obtida de $r$ substituindo cada $x$ por $\tilde{x}$. Agora, $\vartheta$ anula cada $\tilde{r}$, e então para cada $r \in R, \tilde{r} \in k e r \vartheta=I m l$ e desde que $I m l$ é gerada pelo conjunto $\tilde{Y}$, cada $\tilde{r}$ pode ser escrito como uma palavra, digamos, $\vartheta_{r}$ em $\tilde{Y}$. Seja $\tilde{R}=\left\{\tilde{r} \vartheta_{r}^{-1} ; r \in R\right\}$.

Finalmente, como Iml é um subgrupo normal de $\tilde{G}$, cada conjugado $\tilde{x}^{-1} \tilde{y} \tilde{x}, \tilde{x} \in$ $\tilde{X}, \tilde{y} \in \tilde{Y}$ pertence a $\operatorname{Iml}$ e assim é uma palavra, digamos, $w_{x, y}$ em $\tilde{Y}$. Seja $\tilde{T}=$ $\left\{\tilde{x}^{-1} \tilde{y} \tilde{x} w_{x, y}^{-1} ; x \in X, y \in Y\right\}$, e temos o seguinte resultado:

Teorema 2.3.5. Com as notações anteriores, o grupo $\tilde{G}$ tem como apresentação:

$$
<\tilde{X}, \tilde{Y} ; \tilde{R}, \tilde{S}, \tilde{T}>
$$

Demonstração: Seja $D$ o grupo que tem a apresentação $<\tilde{X}, \tilde{Y} ; \tilde{R}, \tilde{S}, \tilde{T}>$. Vamos mostrar que $\tilde{G}$ é isomorfo a $D$. Para isso, usaremos o Lema dos Cinco que provamos a pouco.

Sendo $D=<\tilde{X}, \tilde{Y} ; \tilde{R}, \tilde{S}, \tilde{T}>$, temos, pelo teorema de Von Dick, que existe um homomorfismo $\theta: D \rightarrow \tilde{G}$ dado por $\theta(\tilde{x})=\tilde{x}$ e $\theta(\tilde{y})=\tilde{y}$.

A restrição de $\theta$ ao subgrupo $<\tilde{Y}>$ de $D$ dá origem a um homomorfismo

$$
\theta_{1}:<\tilde{Y}>\rightarrow \operatorname{Iml}(\simeq A)
$$

dado por $\theta_{1}(\tilde{y})=y$. 
Como as relações definidoras $S$ de $A$ (com cada $y$ substituído por $\tilde{y}$ ) são satisfeitas em $<\tilde{Y}>\leq D$, então $\theta_{1}$ é uma bijeção.

A presença das relações $\tilde{T}$ na apresentação de $D$ nos diz que $<\tilde{Y}>$ é um subgrupo normal de $D$ e, desde que $\theta(<\tilde{Y}>) \leq I m l, \theta$ induz um homomorfismo:

$$
\theta_{2}: \frac{D}{<\tilde{Y}>} \rightarrow \frac{\tilde{G}}{I m l}(\simeq G)
$$

dado por $\theta_{2}(\tilde{x}<\tilde{Y}>)=x$.

Agora, as relações $R$ definidas em $G$ são satisfeitas (substituindo $x$ por $\tilde{x}<\tilde{Y}>$ ) em $\frac{D}{<\tilde{Y}>}$, então $\theta_{2}$ deve ser uma bijeção. Desta forma, temos o seguinte diagrama comutativo:

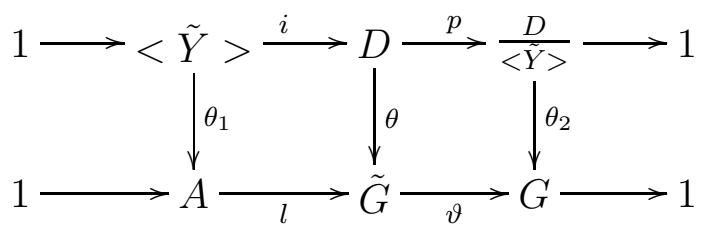

com linhas exatas. Como $\theta_{1}$ e $\theta_{2}$ são isomorfismos, o resultado segue pelo Lema dos Cinco.

Corolário 2.3.6. Sejam $G=<X ; R>$ e $A=<Y$; $S>$ grupos $\alpha: G \rightarrow$ AutA um homomorfismo tal que $\alpha(x)(y)=w_{x, y}$, onde $w_{x, y}$ é uma palavra em $Y^{ \pm 1}$, com $x \in X, y \in$ Y. Então o produto semidireto tem a seguinte apresentação:

$$
A] G=<X, Y ; R, S,\left\{x^{-1} y x w_{x, y}^{-1} ; x \in X, y \in Y\right\}>
$$

Corolário 2.3.7. Seja $\tilde{G}$ uma extensão de $G$ por $A$. Se $G$ e A são finitamente apresentados, então $\tilde{G}$ também será finitamente apresentado.

\subsubsection{Casos especiais}

Como havíamos dito anteriormente, a ideia de uma extensão de grupos é muito geral. Existem quatro casos particulares favoráveis e muito úteis que vamos discutir agora. Para isso, vamos considerar a extensão:

$$
1 \longrightarrow A \stackrel{l}{\longrightarrow} \tilde{G} \stackrel{\vartheta}{\longrightarrow} G \longrightarrow 1
$$


e sua apresentação $<\tilde{X}, \tilde{Y} ; \tilde{R}, \tilde{S}, \tilde{T}>$.

\section{Produtos semidiretos}

Este é o caso em que $N=I m l$ tem um complemento, que chamamos de $C$ em $\tilde{G}$, que é, um subgrupo $C \leq \tilde{G}$ tal que

$$
\tilde{G}=N C, \quad N \cap C=1 \quad(*)
$$

então $\tilde{G}$ é um produto semidireto $A] C$. Por $(*)$, os elementos de $C$ formam uma transversal à direita para $N$ em $\tilde{G}$, e segue que a restrição $\left.\vartheta\right|_{C}$ é um isomorfismo. Então, existe um homomorfismo $\sigma$ :

$$
G \stackrel{\left(\left.\vartheta\right|_{C}\right)^{-1}}{\longrightarrow} C \stackrel{i}{\longrightarrow} \tilde{G}
$$

onde $i: C \rightarrow \tilde{G}$ é o homomorfismo inclusão e $\sigma$ é tal que $\sigma \circ \vartheta=I d_{G}$ (tal homomorfismo $\sigma$ é chamado uma cisão para a extensão $1 \longrightarrow A \stackrel{l}{\longrightarrow} \tilde{G} \stackrel{\vartheta}{\longrightarrow} G \longrightarrow 1$ ).

Reciprocamente, se $1 \longrightarrow A \stackrel{l}{\longrightarrow} \tilde{G} \stackrel{\vartheta}{\longrightarrow} G \longrightarrow 1$ é cindida por $\sigma: G \rightarrow \tilde{G}$ (isto é, $\sigma$ é um homomorfismo tal que $\left.\sigma \circ \vartheta=I d_{G}\right)$, então $I m \sigma$ é claramente um complemento para $N$ em $\tilde{G}$. Assim o produto semidireto nada mais é que uma extenção cindida.

No caso em que $1 \longrightarrow A \stackrel{l}{\longrightarrow} \tilde{G} \stackrel{\vartheta}{\longrightarrow} G \longrightarrow 1$ é cindida, por digamos $\sigma$, podemos escolher os geradores $\tilde{X}$ na prova do Teorema 2.3 .5 como sendo $\{\sigma(x) ; x \in X\}$. Então, para cada $r \in R$, temos $\tilde{r}=\sigma(r)=1$, e, em $\tilde{R}$, todos os $\vartheta_{r}$ são iguais a 1 . Extensões cindidas de $G$ por $A$ são então parametrizadas por $w_{x, y}, x \in X, y \in Y$ apenas. O automorfismo $\alpha$ que define o correspondente produto semidireto $A] G$ é então dado por: $\alpha: G \rightarrow A u t A$, $\operatorname{com} \alpha(x)(y)=w_{x, y}$.

\section{Extensões com núcleo abeliano}

$A$ é geralmente chamado de núcleo da extensão $1 \longrightarrow A \stackrel{l}{\longrightarrow} \tilde{G} \stackrel{\vartheta}{\longrightarrow} G \longrightarrow 1$. Como $A$ é subgrupo normal de $\tilde{G}$, existe um homomorfismo $\gamma: \tilde{G} \rightarrow A u t A$ induzido por conjugação. Então a aplicação $\gamma \circ l: A \rightarrow A u t A$ é induzida por conjugação em $A$, e é trivial se, e somente se, $A$ for abeliano. Neste caso, $\gamma$ induz um homomorfismo $\alpha: G \rightarrow A u t A$.

\section{Extensões Centrais}


Este é o caso em que $N=I m l$ está contido no centro de $\tilde{G}$. Então, não apenas $A$ é abeliano, mas o homomorfismo $\gamma$ definido acima é o trivial. Quando isso acontece, os $w_{x, y}$ que aparecem nos relatores $\tilde{T}$ são os mais simples possíveis, ou seja, $w_{x, y}=\tilde{y}$, para todo $x \in X, y \in Y$. Extensões centrais são portanto, parametrizadas pelos $\vartheta_{r}, r \in R$ apenas. Em outras palavras, $G$ é determinado por $|R|$ escolhas de um conjunto com $|A|$ elementos, e assim, temos o seguinte lema:

Lema 2.3.8. O número total de extensões de um grupo $G=<X ; R>$ por um grupo $A$ é no máximo $|A|^{|R|}$.

\section{Produto Direto}

Suponhamos os dois casos anteriores ocorrendo ao mesmo tempo, isto é, a extensão sendo central com núcleo abeliano, ou seja, a extensão $1 \longrightarrow A \stackrel{l}{\longrightarrow} \tilde{G} \stackrel{\vartheta}{\longrightarrow} G \longrightarrow 1$ é cindida central. Então os $\vartheta_{r}$ na apresentação de $\tilde{G}$ são iguais a 1 e $w_{x, y}=\tilde{y}$, de modo que a apresentação no Teorema 2.3.5 reduz-se a apresentação do produto direto $A \times G$. Isto acontece se, e somente se existir um homomorfismo $\tau: \tilde{G} \rightarrow A$ tal que $\tau \circ l=I d_{A}$. 


\section{Capítulo}

\section{3}

\section{Estrutura do grupo fundamental de uma superfície compacta}

O nosso objetivo neste capítulo será calcular o grupo fundamental das seguintes superfícies: toro, soma conexa de n-toros (casos orientáveis), plano projetivo e soma conexa de n-planos projetivos (casos não-orientáveis). Para isto, vamos enunciar alguns teoremas muito importantes como o Teorema da Classificação de Superfícies e o Teorema de Seifert e Van Kampen.

Definição 3.0.9. Um subconjunto $A$ de um espaço topológico $X$ é chamado retrato de $X$ se existir uma aplicação contínua $r: X \rightarrow A$ (chamada retração) tal que $r(a)=a$, para cada $a \in A$.

Definição 3.0.10. Um subconjunto $A$ de um espaço topológico $X$ é um retrato por deformação de $X$ se existir uma retração $r: X \rightarrow A$ e uma homotopia $f: X \times I \rightarrow X$ tal que $f(x, 0)=x, f(x, 1)=r(x)$, para todo $x \in X$ e $f(a, t)=a$, para cada $a \in A$ e cada $t \in I$, onde $I=[0,1]$.

Definição 3.0.11. Uma superfície que é soma conexa de $n$-toros ou $n$-planos projetivos é dita ser de gênero $n$, enquanto a esfera é de gênero 0. 


\subsection{Teoremas}

Teorema 3.1.1. (Teorema da Classificação de Superfícies) Qualquer superfície compacta é homeomorfa a uma esfera, ou a uma soma conexa de toros, ou uma soma conexa de planos projetivos.

Teorema 3.1.2. Seja A um subconjunto de um espaço topológico $X$. Se A é um retrato por deformação de $X$, então a aplicação inclusão $i: A \rightarrow X$ induz um isomorfismo de $\pi_{1}(A, a)$ sobre $\pi_{1}(X, a)$, para todo $a \in A$.

Teorema 3.1.3. O grupo fundamental do produto de dois espaços topológicos $X$ e $Y$ é isomorfo ao produto direto de seus grupos fundamentais. Em simbolos, $\pi_{1}(X \times Y) \simeq$ $\pi_{1}(X) \times \pi_{1}(Y)$.

Teorema 3.1.4. Se $X$ e $Y$ forem espaços topológicos conexos por caminhos que possuem o mesmo tipo de homotopia então seus grupos fundamentais serão isomorfos, isto é, $\pi_{1}(X) \simeq \pi_{1}(Y)$.

Teorema 3.1.5. Se $X$ for conexo por caminhos então os grupos $\pi_{1}(X, x)$ e $\pi_{1}(X, y)$ são isomorfos para todo $x, y \in X$.

Agora, vamos considerar $X$ um espaço topológico conexo por caminhos, $U, V$ subconjuntos abertos, conexos por caminhos de $\mathrm{X}$ tais que $X=U \cup V$ e $U \cap V$ é não-vazio e conexo por caminhos. Vamos escolher também um ponto base $x_{0} \in X$ tal que $x_{0} \in U \cap V$.

Teorema 3.1.6. (Teorema de Seifert-Van Kampen - versão 1) Considere $\left(X, x_{0}\right)$ um espaço topológico com ponto base $x_{0}$. Sejam $U, V \subset X$ abertos conexos por caminhos tal que $x_{0} \in U \cap V$ e $U \cap V$ é conexo por caminhos, $X=U \cup V$. Então as inclusões naturais induzem um quadrado push-out de grupos:

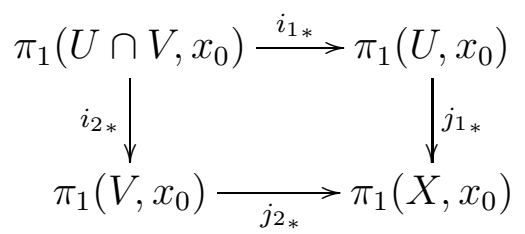


Corolário 3.1.7. (Teorema de Seifert-Van Kampen - versão 2) Nas mesmas hipóteses do teorema anterior e assumindo que $V$ seja simplesmente conexo. Então, $\psi_{1}: \pi_{1}(U) \rightarrow$ $\pi_{1}(X)$ é um epimorfismo e seu kernel é o menor subgrupo normal de $\pi_{1}(U)$ contendo a imagem $\varphi_{1}\left[\pi_{1}(U \cap V)\right]$.

Teorema 3.1.8. (Teorema de Seifert-Van Kampen - versão 3) Se $\left(X, x_{0}\right)$ é um espaço topológico com ponto base $x_{0}$ tal que $X=U \cup V$, onde $U, V$ são abertos conexos por caminhos, $U \cap V$ é conexo por caminhos e $x_{0} \in U \cap V$. Então, $\pi_{1}\left(X, x_{0}\right)$ é isomorfo a $\frac{\pi_{1}\left(U, x_{0}\right) * \pi_{1}\left(V, x_{0}\right)}{N}$, onde $N$ é o subgrupo normal de $\pi_{1}\left(X, x_{0}\right)$ gerado pela palavra $\left(i_{1} *[\alpha]\right) *$ $\left(i_{2} *[\alpha]\right)^{-1}$, para cada $[\alpha] \in \pi_{1}\left(U \cap V, x_{0}\right)$, onde $i_{1}: U \cap V \hookrightarrow U$ e $i_{2}: u \cap V \hookrightarrow V$ são as inclusões naturais.

As demonstrações dos teoremas enunciados acima podem ser encontrados em [M] e [C]. Vamos agora, mostrar por meio de exemplos como a versão 2 do Teorema de SeifertVan Kampen pode ser usado para determinar a estrutura do grupo fundamental de várias 2 -variedades conexas e compactas.

\section{$3.2 \mathrm{O}$ grupo fundamental de algumas superfícies com- pactas orientáveis}

\subsubsection{O toro 2-dimensional $\mathrm{T}$}

" O grupo fundamental do toro 2 -dimensional $\pi_{1}\left(\boldsymbol{T}, x_{0}\right)$ é um grupo livre gerado por 2 elementos."

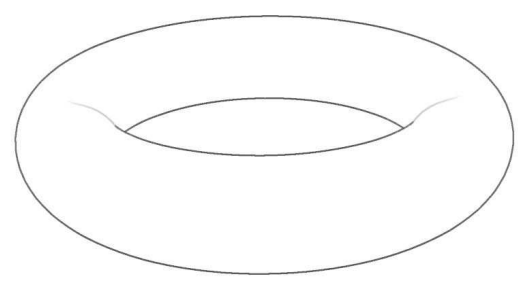

Figura 3.1: $X=\mathbf{T}$

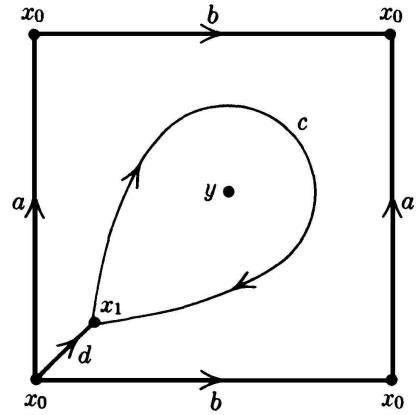

Figura 3.2: Identificação do toro 
Temos $\mathbf{T}=S^{1} \times S^{1}$. Logo, pelo Teorema 3.1.3, $\pi_{1}(\mathbf{T}) \simeq \pi_{1}\left(S^{1}\right) \times \pi_{1}\left(S^{1}\right)$ é o produto de dois grupos cíclicos infinitos, isto é, um grupo abeliano com dois geradores. Contudo, vamos obter esse resultado usando a versção 2 do Teorema de Seifert-Van Kampen.

Considere a identificação do toro como na Figura 3.2. Assim, os lados $a$ e $b$ tornam- se círculos que se interceptam em $x_{0}$. Sejam $y$ o ponto no centro do quadrado, $U=\mathbf{T} \backslash\{y\}$, $V$ a imagem do interior do quadrado e a interseção de $U$ e $V$ como na Figura 3.3 abaixo:
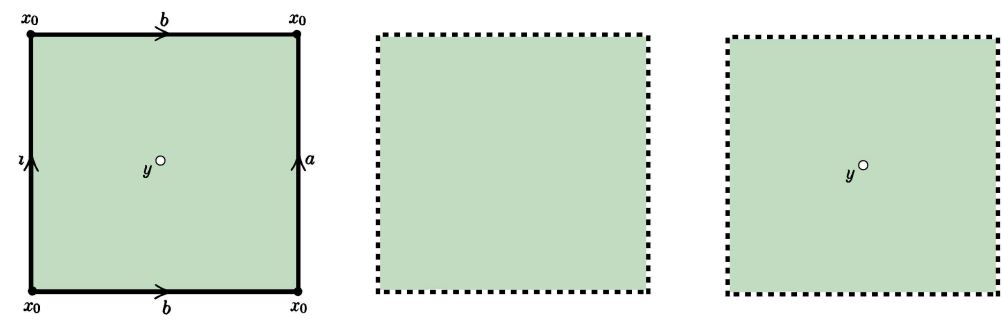

Figura 3.3: Os abertos $U, V$ e $U \cap V$

Desta forma, $U$ e $V$ são abertos, conexos por caminhos, e desde que $V$ tem o mesmo tipo de homotopia do disco, segue que $V$ é simplesmente conexo. Assim, estamos nas hipóteses da versão 2 do Teorema de Seifert-Van Kampen. Observe que o Teorema 3.1.5 nos permite considerar o ponto base $x_{1}$ que está na interseção de $U$ e $V$. Logo, temos que $\psi_{1}: \pi_{1}\left(U, x_{1}\right) \rightarrow \pi_{1}\left(\mathbf{T}, x_{1}\right)$ é um epimorfismo e o núcleo de $\psi_{1}$ é o menor subgrupo normal contendo a imagem do homomorfismo $\varphi_{1}: \pi_{1}\left(U \cap V, x_{1}\right) \rightarrow \pi_{1}\left(U, x_{1}\right)$.

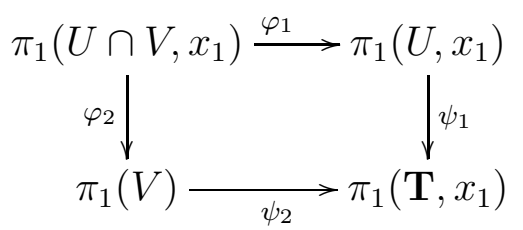

Desde que a fronteira do quadrado é um retrato de deformação do quadrado todo menos um ponto, temos que a união de dois círculos $a$ e $b$ é um retrato de deformação de $U$. Desta forma, o Teorema 3.1.2 nos garante que o grupo $\pi_{1}\left(U, x_{1}\right)$ é um grupo livre com dois geradores. Mais precisamente, $\pi_{1}\left(U, x_{0}\right)$ é um grupo livre com dois geradores $\alpha$ e $\beta$ onde tais geradores são representados pelos círculos $a$ e $b$ respectivamente.

Então $\pi_{1}\left(U, x_{1}\right)$ é um grupo livre sobre os dois geradores:

$$
\begin{aligned}
& \alpha^{\prime}=\delta^{-1} \alpha \delta \\
& \beta^{\prime}=\delta^{-1} \beta \delta,
\end{aligned}
$$

onde $\delta$ é a classe de equivalência de um caminho $d$ de $x_{0}$ a $x_{1}$. Claramente, $U \cap V$ possui o mesmo tipo de homotopia de um círculo. Portanto $\pi_{1}\left(U \cap V, x_{1}\right)$ é um grupo 
cíclico infinito gerado por $\gamma$, onde $\gamma$ representa a classe de equivalência de um caminho fechado $c$, representado na figura 3.2 ao redor de $y$. Também, pela Figura 3.2 segue que $\varphi(\gamma)=\alpha^{\prime} \beta^{\prime} \alpha^{\prime-1} \beta^{\prime-1}$.

Por outro lado, temos que $V$ possui o mesmo tipo de homotopia de um ponto. Logo, $\pi_{1}\left(V, x_{1}\right)=1$. E também, pelo Teorema dos isomorfismos, $\operatorname{Im} \varphi_{1}$ é isomorfo a $\frac{\pi_{1}\left(U, x_{1}\right)}{\left\langle\varphi_{1}(U \cap V)>^{\pi_{1}\left(U, x_{1}\right)}\right.}$. Portanto, temos $\pi_{1}\left(\mathbf{T}, x_{1}\right)$ é isomorfo a $\frac{\pi_{1}\left(U, x_{1}\right)}{<\alpha^{\prime} \beta^{\prime} \alpha^{\prime-1} \beta^{\prime-1}>^{\pi_{1}\left(U, x_{1}\right)}}$, e assim:

$$
\varphi\left(\pi_{1}\left(U \cap V, x_{1}\right)\right) \subset<\alpha^{\prime} \beta^{\prime} \alpha^{\prime-1} \beta^{\prime-1}>^{\pi_{1}\left(U, x_{1}\right)}=\operatorname{ker} \varphi_{1} .
$$

$\operatorname{Logo}, \varphi_{1}(\gamma)=\alpha^{\prime} \beta^{\prime} \alpha^{\prime-1} \beta^{\prime-1}=1$, ou seja, $\alpha^{\prime} \beta^{\prime}=\beta^{\prime} \alpha^{\prime}$. Portanto, $\pi_{1}\left(\mathbf{T}, x_{1}\right)$ é um grupo livre abeliano gerado por $\left\{\alpha^{\prime}, \beta^{\prime}\right\}$, e assim segue que $\pi_{1}\left(\mathbf{T}, x_{0}\right)$ é um grupo abeliano livre gerado por $\{\alpha, \beta\}$.

\subsubsection{Soma conexa de n-toros}

Com raciocínio análogo ao anterior, vamos calcular o grupo fundamental da soma conexa de $n$-toros $\pi_{1}\left(\mathbf{M}, x_{0}\right)$.

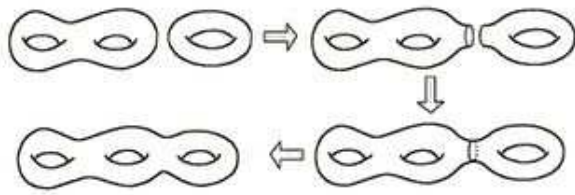

Figura 3.4: Soma conexa de toros

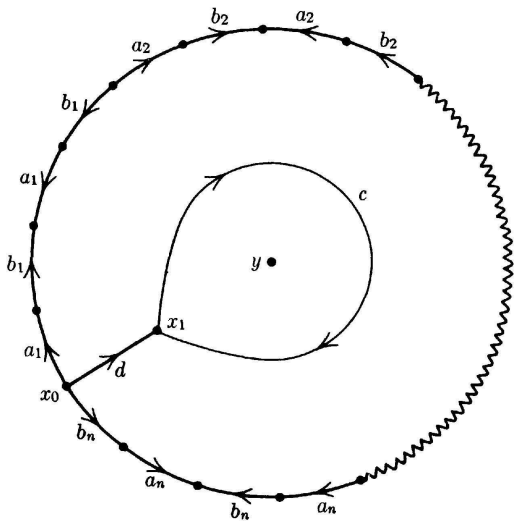

Figura 3.5: Identificação da soma conexa de toros

Vamos chamar a soma conexa de n-toros de $\mathbf{M}$ e sua representação é dada por um 4n-polígono com os lados identificados em pares, como mostra a Figura 3.5. Sob esta identificação, os arcos $a_{1}, b_{1}, a_{2}, b_{2}, \ldots, a_{n}, b_{n}$ tornam-se círculos em $\mathbf{M}$ e, quaisquer dois destes círculos se interceptam apenas no ponto base $x_{0}$. Vamos tomar os seguintes abertos: $U=\mathbf{M} \backslash\{y\}$, o complemento do ponto central $y$, assim $V$ é a imagem do interior do polígono (por um disco aberto em $\mathbf{M}$ ). A união de $2 n$ círculos $a_{1}, b_{1}, a_{2}, b_{2}, \ldots, a_{n}, b_{n}$ é um retrato por deformação de $U$ e, portanto, $\pi_{1}\left(U, x_{0}\right)$ é um grupo livre de $2 n$ geradores, a saber, $\alpha_{1}, \beta_{1}, \ldots, \alpha_{n}, \beta_{n}$, onde $\alpha_{i}$ é representado por $a_{i}$ e $\beta_{i}$ é representado por $b_{i}$. Como 
antes, $\pi_{1}\left(U \cap V, x_{1}\right)$ é um grupo cíclico infinito com gerador $\gamma$ representado pelo círculo $c$ e $\varphi_{1}(\gamma)=\prod_{i=1}^{n}\left[\alpha_{i}^{\prime}, \beta_{i}^{\prime}\right]$, onde $\left[\alpha_{i}^{\prime}, \beta_{i}^{\prime}\right]=\alpha_{i}^{\prime} \beta_{i}^{\prime} \alpha_{i}^{\prime-1} \beta_{i}^{\prime-1}, \operatorname{com} \alpha_{i}^{\prime}=\delta^{-1} \alpha_{i} \delta, \beta_{i}^{\prime}=\delta^{-1} \beta_{i} \delta$, sendo $\delta$ a classe de equivalência do caminho $d$.

Consideremos também o seguinte push-out:

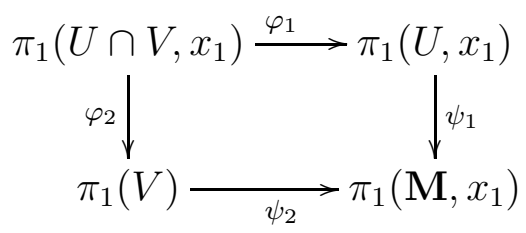

Assim, concluímos que $\pi_{1}\left(\mathbf{M}, x_{0}\right)$ é o quociente de um grupo livre nos geradores $\alpha_{1}, \beta_{1}, \ldots, \alpha_{n}, \beta_{n}$ pelo subgrupo normal gerado por $\prod_{i=1}^{n}\left[\alpha_{i}, \beta_{i}\right]$, ou seja,

$$
\pi_{1}\left(\mathbf{M}, x_{0}\right)=<\alpha_{1}, \beta_{1}, \ldots, \alpha_{n}, \beta_{n} ; \prod_{i=1}^{n}\left[\alpha_{i}, \beta_{i}\right]>
$$

Observemos que se "abelianizarmos" $\pi_{1}\left(\mathbf{M}, x_{0}\right)$ (isto é, fazendo o quociente de $\pi_{1}\left(\mathbf{M}, x_{0}\right)$ pelo seu subgrupo dos comutadores) vamos obter um grupo livre abeliano de $2 n$ geradores.

Esta é uma consequência da única relação obtida que está contida no subgrupo dos comutadores do grupo livre gerado por $\alpha_{1}, \beta_{1}, \ldots, \alpha_{n}, \beta_{n}$. Disto segue que se $m \neq n$, não há isomorfismo entre a soma conexa de n-toros e a soma conexa de m-toros e, pelo Teorema 3.1.4, elas não possuem o mesmo tipo de homotopia.

\subsection{O grupo fundamental de algumas superfícies não ori-}

\section{entáveis}

\subsubsection{O plano real projetivo $P_{2}(\mathbb{R})$}

O grupo fundamental do plano projetivo $\pi_{1}\left(P_{2}(\mathbb{R})\right)$ é um grupo cíclico de ordem 2 . De fato, consideremos $P_{2}(\mathbb{R})$ o espaço obtido identificando os lados opostos de um polígono de 2 lados como mostra a Figura 3.6 abaixo: 


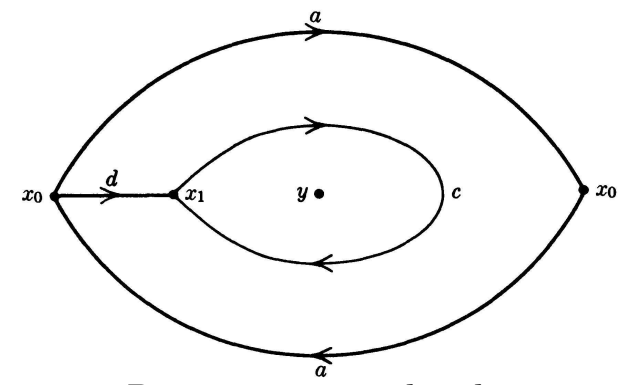

Figura 3.6: Representação do plano projetivo.

Considerando a identificação acima, a aresta $a$ torna-se um círculo. Sejam y o ponto no centro do polígono, $U=P_{2}(\mathbb{R}) \backslash\{y\}$ e $V$ a imagem do interior do polígono sob a identificação.
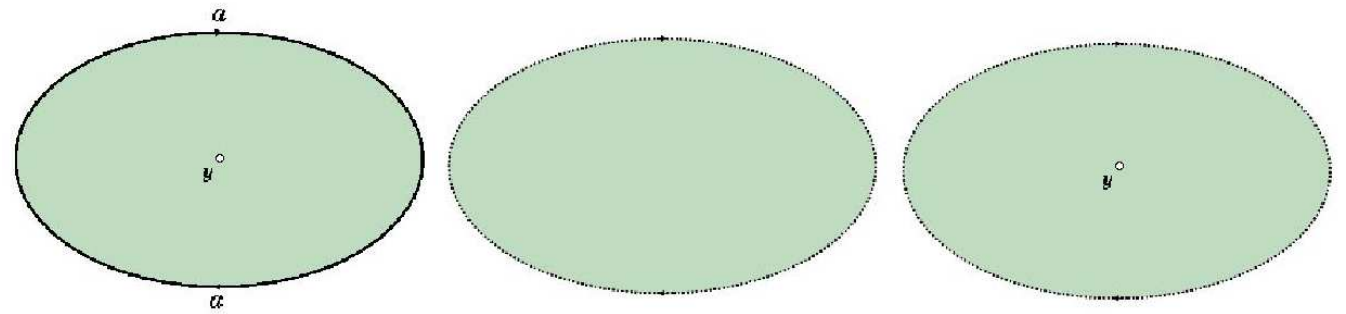

Figura 3.7: Os abertos $U, V$ e $U \cap V$

Desde que $V$ possui o mesmo tipo de homotopia de um ponto, segue que $V$ é simplesmente conexo e, assim $\pi_{1}\left(V, x_{1}\right)=1$. E também estamos nas condições do Corolário 3.1.7. Neste caso, o círculo a é um retrato por deformação de $U$, portanto, $\pi_{1}\left(U, x_{0}\right)$ é um grupo cíclico infinito gerado por $\alpha$ representado pelo caminho fechado $a$. E também, $\pi_{1}\left(U, x_{1}\right)$ é um grupo cíclico infinito gerado por $\alpha^{\prime}=\delta^{-1} \alpha \delta$, com $\delta$ sendo a classe de equivalência do caminho $d$. Finalmente, $\pi_{1}\left(U \cap V, x_{1}\right)$ é um grupo cíclico finito com gerador $\gamma$ que representa o caminho fechado $c$ que está ao redor de $y$.

Claramente, temos $\varphi_{1}(\gamma)=\alpha^{\prime 2}$. Portanto, $\pi_{1}\left(P_{2}(\mathbb{R}), x_{1}\right)$ é o quociente de um grupo cíclico infinito gerado por $\alpha^{\prime}$ pelo subgrupo gerado por $\alpha^{\prime 2}$. Logo, $\pi_{1}\left(P_{2}(\mathbb{R}), x_{0}\right)$ também é quociente de um grupo cíclico infinito gerado por $\alpha$ pelo subgrupo gerado por $\alpha^{2}$ e portanto, $\pi_{1}\left(P_{2}(\mathbb{R}), x_{0}\right)$ é um grupo cíclico de ordem 2 .

\subsubsection{Soma conexa de n-planos projetivos}

Aqui, vamos analisar dois casos para o grupo fundamental da soma conexa de $n$-planos projetivos: o caso em que $n$ for par e o caso em que $n$ for ímpar. Seja $\mathbf{M}$ a soma conexa 
de n-planos projetivos. Temos que $\mathbf{M}$ pode ser obtido identificando em pares os lados de um $2 n$-polígono como mostra a Figura 3.8 abaixo:

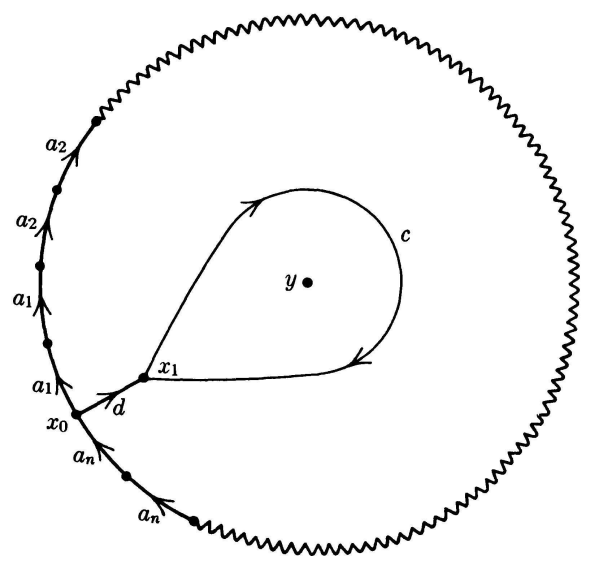

Figura 3.8: Representação da soma conexa de n-planos projetivos.

Procedendo como anteriormente, encontramos o grupo fundamental com apresentação consistindo dos geradores $\left\{\alpha_{1}, \ldots, \alpha_{n}\right\}$ onde cada $\alpha_{i}$ é representado pelo círculo $a_{i}$ e a relação $\alpha_{1}^{2} \alpha_{2}^{2} \ldots \alpha_{n}^{2}$, ou seja,

$$
\pi_{1}\left(\mathbf{M}, x_{0}\right)=<\left\{\alpha_{1}, \ldots, \alpha_{n}\right\} ; \alpha_{1}^{2} \alpha_{2}^{2} \ldots \alpha_{n}^{2}>
$$

Observemos que se "abelianizarmos" $\pi_{1}\left(\mathbf{M}, x_{0}\right)$, obteremos um grupo abeliano que também possui uma apresentação com $n$ geradores e uma relação. 


\section{Capítulo}

4

\section{Introdução aos grupos das tranças no}

\section{disco}

Na primeira seção deste capítulo vamos definir os Grupos de Tranças no Disco e ver uma apresentação para o mesmo. Depois estudaremos os Grupos de Tranças em Superfícies e veremos que o grupo de tranças no disco é um caso particular deste.

\subsection{Tranças Geométricas}

Vamos denotar por $\mathbb{E}^{3}$ o espaço euclidiano. Vamos identificá-lo com o espaço real de dimensão $3, \mathbb{R}^{3}$, escolhendo um sistema de coordenadas $(x, y, z)$ no qual o eixo $Z$ está orientado pra baixo como mostra a Figura 4.1. Vamos considerar também, dois planos paralelos em $\mathbb{E}^{3}$ nas constantes $z=z_{0}$ e $z=z_{1}$, onde $z_{0}<z_{1}$. Chamaremos os planos $z=z_{0}$ de plano superior e $z=z_{1}$ de plano inferior. Vamos marcar $n$ pontos distintos $P_{1}, \ldots, P_{n}$ numa reta no plano superior e projetá-los ortogonalmente sobre o plano inferior nos pontos $P_{1}^{\prime}, \ldots, P_{n}^{\prime}$.

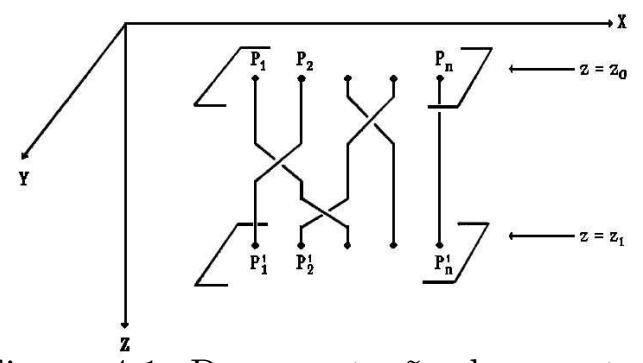

Figura 4.1: Representação de uma trança. 
Definição 4.1.1. Uma trança geométrica de $\mathrm{n}$-cordas $\beta$ é um sistema de arcos mergulha$\operatorname{dos} \mathscr{A}=\left\{\mathscr{A}_{1}, \ldots, \mathscr{A}_{n}\right\}$ em $\mathbb{E}^{3}$, onde o $i$-ésimo arco $\mathscr{A}_{i}$ conecta o ponto $P_{i}$ do plano superior com o ponto $P_{\tau(i)}^{\prime}$ no plano inferior para alguma permutação $\tau$ de $\{1, \ldots, n\}$, satisfazendo:

(i) Cada arco $\mathscr{A}_{i}$ intercepta cada plano paralelo intermediário entre os planos superior e inferior exatamente uma vez;

(ii) Os arcos $\left\{\mathscr{A}_{1}, \ldots, \mathscr{A}_{n}\right\}$ interceptam cada plano paralelo intermediário entre os planos superior e inferior em exatamente $n$ pontos distintos.

A permutação $\tau$ é chamada permutação da trança. O arco $\mathscr{A}_{i}$ é chamado de i-ésima corda na trança.

Observação 4.1.2. Podemos pensar num arco em $\mathbb{E}^{3}$ como a imagem de um mergulho $\mathscr{A}_{i}:[0,1] \rightarrow \mathbb{E}^{3}$. Usaremos a mesma notação para o arco e seu mergulho correspondente.

Vamos introduzir um conceito de equivalência de tranças.

Definição 4.1.3. Duas n-tranças $\mathscr{A}^{0}=\left\{\mathscr{A}_{1}^{0}, \ldots, \mathscr{A}_{n}^{0}\right\}$ e $\mathscr{A}^{1}=\left\{\mathscr{A}_{1}^{1}, \ldots, \mathscr{A}_{n}^{1}\right\}$ com a mesma permutação $\tau$ são chamadas equivalentes, se existir uma homotopia entre as tranças geométricas com permutação $\tau$ de $\mathscr{A}^{0}$ a $\mathscr{A}^{1}$, em outras palavras, se existir $n$ aplicações continuas

$$
F_{i}:[0,1] \times[0,1] \rightarrow \mathbb{E}^{3}, 1 \leq i \leq n
$$

satisfazendo

$$
F_{i}(t, 0)=\mathscr{A}_{i}^{0}(t), F_{i}(t, 1)=\mathscr{A}_{i}^{1}(t), 0 \leq t \leq 1,1 \leq i \leq n,
$$

e

$$
F_{i}(0, s)=P_{i}, F_{i}(1, s)=P_{\tau(i)}^{\prime}, 0 \leq s \leq 1,1 \leq i \leq n
$$

e tal que se definirmos $\mathscr{A}_{i}^{s}:[0,1] \rightarrow \mathbb{E}^{3}$ por $\mathscr{A}_{i}^{s}(t)=F_{i}(t, s)$, então $\mathscr{A}^{s}=\left\{\mathscr{A}_{1}^{s}, \ldots, \mathscr{A}_{n}^{s}\right\}$ é uma n-trança geométrica (com permutação $\tau$ ), para cada $0 \leq s \leq 1$.

Podemos assumir, a menos de equivalência, que uma trança $\beta$ consiste apenas de arcos poligonais e que temos cruzamentos transversais dos arcos se projetarmos a trança ortogonalmente sobre o plano em $\mathbb{E}^{3}$ contendo os pontos $P_{1}, \ldots, P_{n}, P_{1}^{\prime}, \ldots, P_{n}^{\prime}$. Esta projeção 
fornece uma figura padrão da trança $\beta$. Também, podemos assumir que os cruzamentos dos arcos ocorrem em diferentes níveis. Tais cruzamentos são indicados como na figura abaixo:

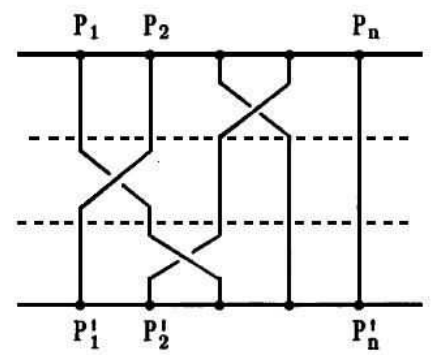

Figura 4.2: Os cruzamentos por baixo e por cima na trança.

Observando a Figura 4.2, verificamos que uma trança pode ser "decomposta" em tranças elementares, que serão definidas a seguir.

Para $1 \leq i \leq n-1$, vamos denotar por $\sigma_{i}$ a $n$-trança geométrica elementar, na qual a $i$-ésima corda cruza por cima a $(i+1)$-ésima corda uma única vez e todas as outras cordas vão do começo ao fim sem se cruzar.

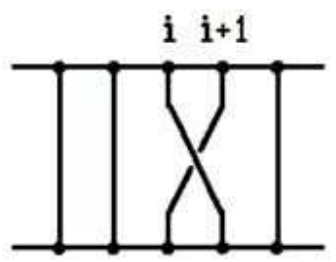

Figura 4.3: A trança elementar $\sigma_{i}$.

Vamos denotar por $B(n)$ o conjunto de todas as classes de equivalência de $n$-tranças geométricas. Este conjunto pode ser equipado com uma estrutura natural de grupo, que vamos definir agora.

Sejam $\beta_{1}, \beta_{2}$ duas $n$-tranças geométricas e vamos definir o produto (composição) de $\beta_{1}$ e $\beta_{2}$, que vamos denotar por $\beta_{1} \beta_{2}$ como segue: primeiro, vamos "grudar" a tranças $\beta_{2}$ embaixo da trança $\beta_{1}$ juntando o plano debaixo de $\beta_{1}$ com o plano de cima de $\beta_{2}$. Então removemos este plano que acabamos de grudar. Agora, comprimimos este novo sistema de cordas até ficarem entre os planos $z=z_{0}$ e $z=z_{1}$. Este é o produto definido entre as tranças $\beta_{1}$ e $\beta_{2}$. 

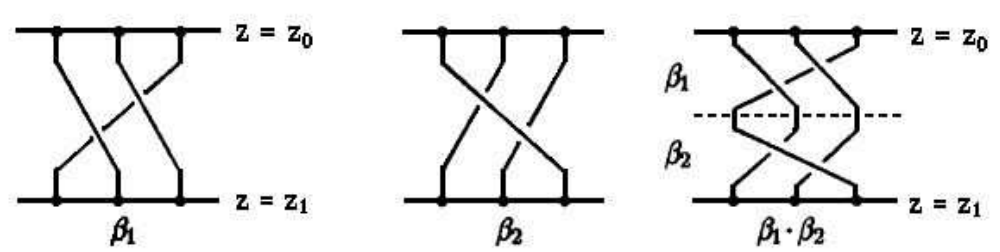

Figura 4.4: O produto entre duas tranças.

Afirmamos agora que, se tomarmos duas tranças $\beta_{1}^{\prime}$ e $\beta_{2}^{\prime}$ equivalentes a $\beta_{1}$ e $\beta_{2}$ respectivamente, então o produto $\beta_{1} \beta_{2}$ será equivalente ao produto $\beta_{1}^{\prime} \beta_{2}^{\prime}$. De fato, sendo $F_{i}$ e $G_{i}, 1 \leq i \leq n$ as $n$ homotopias entre $\beta_{1}$ e $\beta_{1}^{\prime}$ e $\beta_{2}$ e $\beta_{2}^{\prime}$ respectivamente, basta tomarmos as $n$ homotopias $H_{i}: I \times I \rightarrow \mathbb{E}^{3}$ dadas por:

$$
H_{i}(t, s)=\left\{\begin{array}{ll}
F_{i}(2 t, s), & 0 \leq t \leq \frac{1}{2}, s \in I \\
G_{\tau(i)}(2 t-1, s), & \frac{1}{2} \leq t \leq 1, s \in I
\end{array} .\right.
$$

Assim, o produto fica bem definido nas classes de equivalência das $n$-tranças, ou seja, em $B(n)$.

A trança trivial que denotaremos por $\epsilon$ é uma trança na qual todas as cordas apenas vão do começo no plano superior até o fim no plano inferior sem cruzamentos. É fácil ver que a classe de equivalência de $\epsilon$ é o elemento neutro para o produto definido em $B(n)$. A projeção de $\epsilon$ é dada na Figura 4.5:

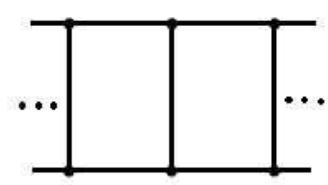

Figura 4.5: A trança trivial.

A trança inversa $\beta^{-1}$ é obtida como a imagem de $\beta$ num espelho com respeito ao plano horizontal entre os planos superior e inferior como se vê na Figura 4.6:

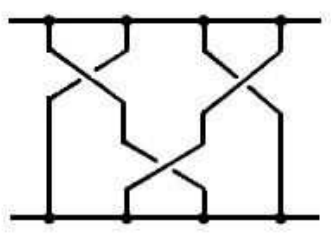

$\beta$

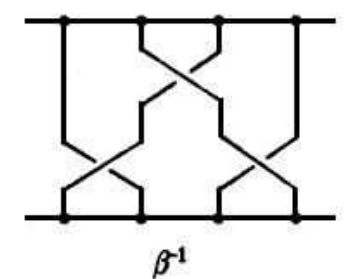

Figura 4.6: A trança inversa. 
A classe de equivalência de $\beta^{-1}$ está bem definida e as tranças produto $\beta \beta^{-1}$ e $\beta^{-1} \beta$ são homotópicas a trança trivial. Portanto, a classe de equivalência de $\beta^{-1}$ é o elemento inverso em $B(n)$ para a classe de equivalência de $\beta$.

Para a trança elementar $\sigma_{i}, 1 \leq i \leq n-1$, a trança $\sigma_{i}^{-1}$ é obtida mudando-se (na projeção padrão) da $i$-ésima corda sobre a $(i+1)$-ésima corda pelo cruzamento da $i$-ésima corda sob a $(i+1)$-ésima corda.

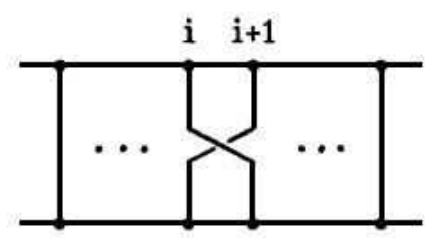

$\sigma_{1}$

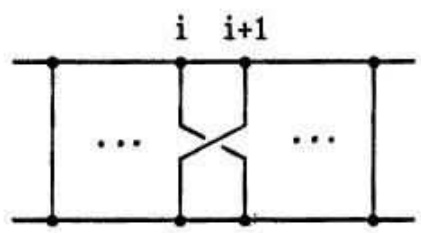

$\sigma_{\mathrm{i}}^{-1}$

Figura 4.7: A trança inversa da trança elementar.

E, com o produto de tranças definido acima, temos que $B(n)$ é um grupo. Este grupo é chamado de Grupo das Tranças de Artin sob n cordas ou simplesmente Grupo das Tranças no Disco. Definimos também o Grupo das Tranças Puras no Disco (subgrupo de B(n)) que é o grupo cujos elementos tem permutação igual a (1), denotado por $P B(n)$.

Observação 4.1.4. A 5-trança $\beta$ da Figura 4.2 pode ser escrita como $\beta=\sigma_{3}^{-1} \sigma_{1}^{-1} \sigma_{2}$. E também, é intuitivo que a classe de equivalência de qualquer n-trança pode ser escrito como um produto de $n$-tranças elementares $\sigma_{i}, 1 \leq i \leq n-1$ e seus inversos. Em outras palavras, as $n$-tranças elementares $\sigma_{1}, \ldots, \sigma_{n}$ geram o grupo $B(n)$.

Vamos olhar agora para algumas relações entre os elementos de $B(n)$. Primeiro, notemos que se $|i-j| \geq 2$ e $1 \leq i, j \leq n-1$, então, desde que o par consistindo das cordas $i$ e $i+1$ não interferem no par consistindo das cordas $j$ e $j+1$, temos a seguinte relação:

$$
\sigma_{i} \sigma_{j}=\sigma_{j} \sigma_{i}, \text { se }|i-j| \geq 2,1 \leq i, j \leq n-1
$$

que é ilustrada abaixo:

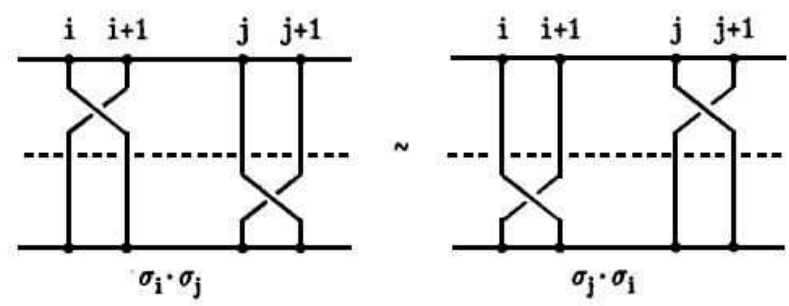

Figura 4.8: Uma relação em $B(n)$. 
Outra relação em $B(n)$ é dada por:

$$
\sigma_{i} \sigma_{i+1} \sigma_{i}=\sigma_{i+1} \sigma_{i} \sigma_{i+1}, \text { se } 1 \leq i \leq n-2
$$

que é ilustrada abaixo:

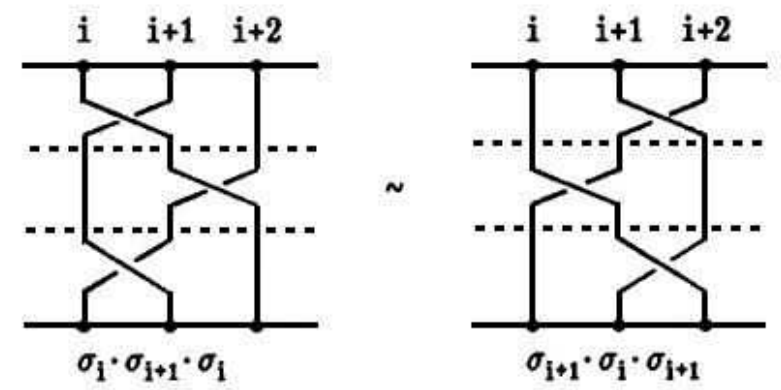

Figura 4.9: Outra relação em $B(n)$.

Teorema 4.1.5. O grupo $B(n)$ das tranças geométricas sob $n$ cordas admite uma apresentação com os seguintes geradores:

$$
\sigma_{1}, \ldots, \sigma_{n-1}
$$

e as seguintes relações:

$$
\begin{aligned}
& \sigma_{i} \sigma_{j}=\sigma_{j} \sigma_{i}, \quad|i-j| \geq 2, \quad 1 \leq i, j \leq n-1 \\
& \sigma_{i} \sigma_{i+1} \sigma_{i}=\sigma_{i+1} \sigma_{i} \sigma_{i+1}, \quad 1 \leq i \leq n-2 .
\end{aligned}
$$

Uma demonstração para este teorema pode ser encontrada em [LH].

Para finalizarmos esta seção de tranças no disco, vamos propor agora uma breve discussão sobre o grupo das tranças puras no disco. Os detalhes podem ser encontrados em BZ].

Definimos as $i$-Tranças Puras $\mathcal{F}^{(i)}$ de $P B(n)$ se para cada $j \neq i$, a corda que sai do ponto $P_{j}$ é a corda trivial e, além disso, a corda que sai de $P_{i}$ pode cruzar somente as cordas que saem de $P_{k}$, com $k=1, \ldots, i-1$. A $i$-trança pura é representada na figura abaixo: 

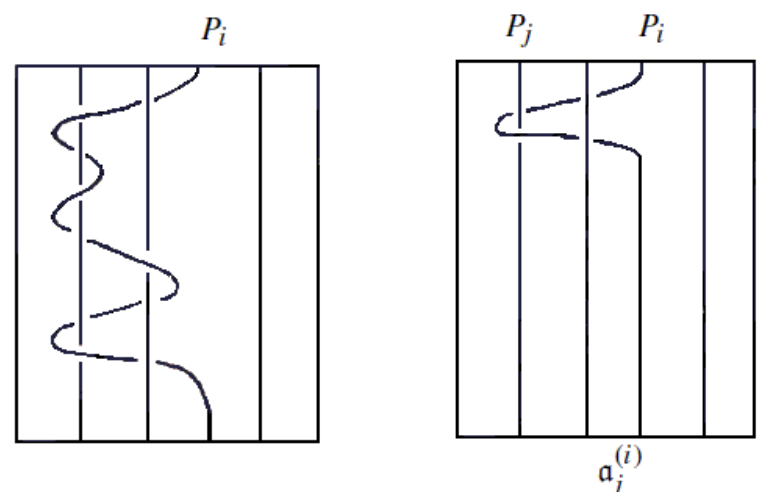

Figura 4.10: A $i$-trança pura denotada por $a_{j}^{(i)}$.

Proposição 4.1.6. As $i$-tranças puras de $P B(n)$ formam um subgrupo livre de posto $i-1$.

Proposição 4.1.7. O subgrupo $B(i-1) \subset B(n)$ gerado pelo conjunto $\left\{\sigma_{r} ; 1 \leq r \leq i-2\right\}$ opera sobre $\mathcal{F}^{(i)}$ por conjugação.

$$
\sigma_{r}^{-1} a_{j}^{(i)} \sigma_{r}= \begin{cases}a_{j}^{(i)}, & j \neq r, r+1, \\ a_{r}^{(i)} a_{r+1}^{(i)} a_{r}^{(i)-1}, & j=r, \\ a_{r}^{(i)}, & j=r+1 .\end{cases}
$$

Proposição 4.1.8. As tranças z de $P B(n)$ admitem uma única decomposição:

$$
z=z_{2} \ldots z_{n}, z_{i} \in \mathcal{F}^{(i)}, \mathcal{F}^{(1)}=1
$$

Esta decomposição é chamada de forma normal de z. Existe uma regra produto para formas normais:

$$
\left(\prod_{i=2}^{n} \zeta_{i}\right)\left(\prod_{i=2}^{n} \eta_{i}\right)=\left(\zeta_{2} \eta_{2}\right)\left(\zeta_{3}^{\lambda_{2}} \eta_{3}\right) \ldots\left(\zeta_{n}^{\lambda_{n-1} \ldots \lambda_{3} \lambda_{2}} \eta_{n}\right)
$$

onde $\lambda_{i}$ denota o automorfismo trança associado à trança $\eta_{i} \in \mathcal{F}^{(i)}$.

O método para pentear uma trança consiste em escrevê-la como produto de $(i)$-tranças puras. Este método é utilizado para resolver o problema da palavra, daí o objetivo da discussão. 


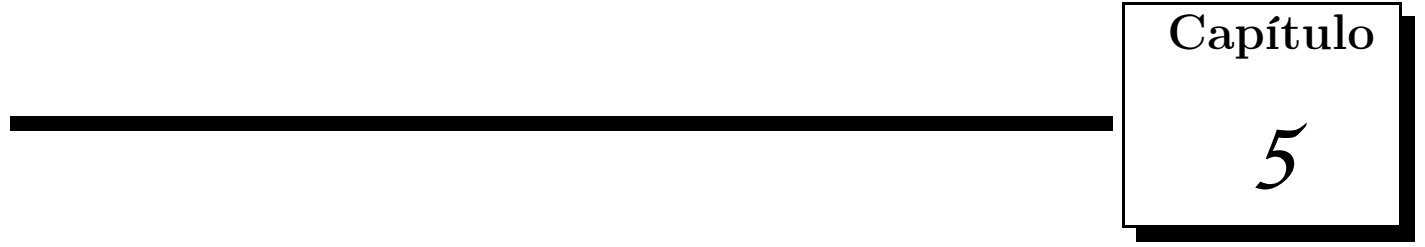 \\ Apresentações dos grupos de tranças em superfícies}

Neste artigo vamos estudar apresentações para o grupo das tranças e o grupo das tranças puras de uma superfície fechada. Também, vamos estudar um algoritmo que resolve o problema da palavra nestes grupos usando as apresentações que encontramos.

\subsection{Introdução}

Seja $M$ uma superfície fechada, não necessariamente orientável, e $\mathcal{P}=\left(P_{1}, \ldots, P_{n}\right)$ um conjunto de $n$ pontos distintos de $M$. Uma trança geométrica em $M$ baseada em $\mathcal{P}$ é uma $n$-upla $\Gamma=\left(\gamma_{1}, \ldots, \gamma_{n}\right)$ de caminhos $\gamma_{i}:[0,1] \rightarrow M$ tal que:

(i) $\gamma_{i}(0)=P_{i}$, para todo $i=1, \ldots, n$;

(ii) $\gamma_{i}(1) \in \mathcal{P}$, para todo $i=1, \ldots, n$;

(iii) $\left\{\gamma_{1}(t), \ldots, \gamma_{n}(t)\right\}$ são $n$ pontos distintos em $M$ para cada $t \in[0,1]$. Para cada $i=1, \ldots, n$ nós dizemos que $\gamma_{i}$ é a i-ésima corda de $\Gamma$.

Duas tranças geométricas baseadas em $\mathcal{P}$ são equivalentes se existir uma homotopia que deforma uma trança na outra de modo que em qualquer tempo sempre tenhamos uma trança geométrica baseada em $\mathcal{P}$. Definimos o produto de duas tranças como o produto induzido pelo produto de caminhos: para cada $i=1, \ldots, n$, compomos a corda da primeira 
trança que termina em $P_{i}$ com a i-ésima corda da segunda trança. Este produto está bem definido e induz no conjunto de classes de equivalência de tranças uma estrutura de grupo. Este grupo é chamado grupo de tranças com $n$ cordas em $M$ baseado em $\mathcal{P}$ e será denotado por $B_{n}(M, \mathcal{P})$. Este grupo não depende, a menos de isomorfismos, da escolha de $\mathcal{P}$, mas depende do número de cordas, então podemos simplificar nossa notação escrevendo apenas $B_{n}(M)$.

Dizemos que uma trança $\Gamma=\left(\gamma_{1}, \ldots, \gamma_{n}\right)$ é pura se $\gamma_{i}(1)=P_{i}$, para cada $i=1, \ldots, n$, isto é, se todas as suas cordas são laços. O conjunto das classes de equivalência das tranças puras é um subgrupo de $B_{n}(M)$ chamado grupo das tranças puras com $n$ cordas em $M$ baseado em $\mathcal{P}$ e vamos denotá-lo por $P B_{n}(M, \mathcal{P})$. Pela mesma razão acima, denotamos este grupo apenas por $P B_{n}(M)$. Claramente, se $n=1$ então $B_{1}(M)=P B_{1}(M)=\pi_{1}(M)$, o grupo fundamental de $M$.

Observação 5.1.1. Se considerarmos o plano $\mathbb{E}^{2}$ como um disco aberto em $M$ e sendo $j: \mathbb{E}^{2} \rightarrow M$ a aplicação inclusão. Então $j$ induz um homomorfismo de grupos $j_{*}: B(n) \rightarrow$ $B_{n}(M)$. Nessas condições temos o seguinte resultado: "Se $M$ é uma superfície fechada exceto $S^{2}$ e $P^{2}$ então $\operatorname{ker} j_{*}=1 "$.

Mais detalhes podem ser encontrados em [B].

Vamos fazer uso de algumas sequências exatas envolvendo grupos de tranças. Considere o grupo simétrico em $n$ elementos $\Sigma_{n}$. A primeira sequência exata será a seguinte:

$$
1 \longrightarrow P B_{n}(M) \stackrel{e}{\longrightarrow} B_{n}(M) \stackrel{f}{\longrightarrow} \Sigma_{n} \longrightarrow 1
$$

onde $e$ é a inclusão natural e $f$ é a aplicação que leva uma dada trança na permutação induzida por ela.

Agora, vamos tomar $\mathcal{P}^{\prime}=\left\{P_{2}, \ldots, P_{n}\right\}$ e considerar $M$ diferente da esfera $S^{2}$ e do plano projetivo $P_{2}$. Temos a seguinte sequência exata:

$$
1 \longrightarrow \pi_{1}\left(M \backslash \mathcal{P}^{\prime}, P_{1}\right) \stackrel{u}{\longrightarrow} P B_{n}(M, \mathcal{P}) \stackrel{\nu}{\longrightarrow} P B_{n-1}\left(M, \mathcal{P}^{\prime}\right) \longrightarrow 1
$$

onde se $\gamma \in \pi_{1}\left(M \backslash \mathcal{P}^{\prime}, P_{1}\right)$ então $u(\gamma)=\left(\gamma, e P_{2}, \ldots, e P_{n}\right)$ onde $e P_{i}$ denota o caminho constante em $P_{i}$ e, para $\Gamma=\left(\gamma_{1}, \ldots, \gamma_{n}\right) \in P B_{n}(M, \mathcal{P})$ temos $\nu(\Gamma)=\left(\gamma_{2}, \ldots, \gamma_{n}\right)$.

Nosso objetivo aqui será determinar apresentações dos grupos de tranças em superfícies fechadas diferentes da esfera e do plano projetivo. Apresentações dos grupos de tranças 
da esfera e do plano projetivo podem ser encontradas em [FvB] e [vB] Vamos mostrar também que estas apresentações fornecerão um algoritmo para solucionar o problema da palavra para grupos de tranças em superfícies.

\subsection{Interpretações geométricas e afirmações}

Nesta seção vamos obter noções geométricas das tranças em superfícies. Mais ainda, vamos conhecer os geradores e as relações dos grupos de tranças e de tranças puras nos casos orientável e não orientável com objetivo de nos familiarizarmos com suas notações e interpretações.

\subsubsection{Caso Orientável}

Vamos supor que $M$ seja uma superfície fechada, orientável, com gênero $g \geq 1$, portanto diferente da esfera. A primeira coisa que queremos ter em mente é uma representação geométrica da trança em $M$. Vamos representar $M$ como um polígono $L$ de $4 g$ lados, identificado como na figura abaixo:

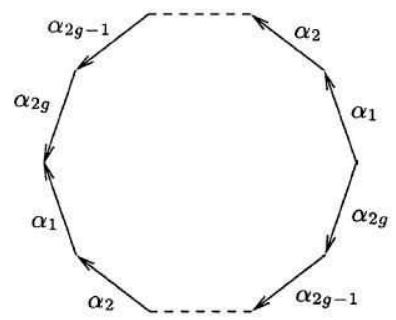

Figura 5.1: Polígono $L$ representando $M$.

Observação 5.2.1. Observemos que a representação acima não é a representação clássica que temos de uma superfície fechada, orientável de gênero $g \geq 1$. Mas afirmamos neste momento que as representações são homeomorfas e, a prova desta afirmação será dada no Apêndice deste mesmo capítulo.

Podemos agora, tomar o cilindro $L \times I$, com $I=[0,1]$, e representar uma trança $\Gamma$ em $M$ como fizemos para as tranças no disco, isto é, em $L \times\{t\}$ desenhamos os $n$ pontos $\gamma_{1}(t), \ldots, \gamma_{2}(t)$. Mas temos uma situação diferente que não ocorre com as tranças no disco: aqui, uma trança pode atravessar alguma parede do cilindro e aparecer do outro lado. Um exemplo disso é o desenho à esquerda na figura seguinte: 

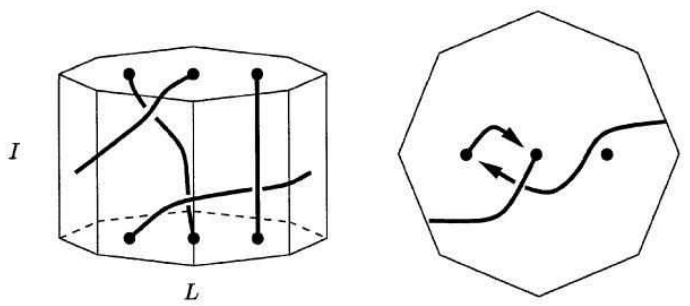

Figura 5.2: Trança numa superfície de gênero 2 vista de maneiras distintas.

Uma outra maneira é olharmos o cilindro por cima, como no desenho à direita na Figura 5.2. Deste modo, conseguimos ver as cordas como caminhos na superfície. Quando duas cordas se cruzam, a que passa por cima da outra é a primeira a chegar no ponto de cruzamento. De qualquer forma, é bom mantermos a idéia que estamos olhando para o cilindro e considerar os caminhos como cordas: desta maneira, podemos ver mais facilmente quando duas tranças geométricas são equivalentes.

Agora podemos definir os geradores de $B_{n}(M)$. Vamos escolher os $n$ pontos base ao longo do diâmetro horizontal de $L$ e, dado $r, 1 \leq r \leq 2 g$, vamos definir a trança $a_{r}$ como segue: a sua única corda não trivial é a primeira, a qual atravessa a $r$-ésima parede indo para cima na figura se $r$ é ímpar e indo para baixo na figura caso contrário.Também, para cada $i=1, \ldots, n-1$ sejam $\sigma_{i}$ as tranças elementares como as que geram o grupo de tranças no disco, vistas no capítulo anterior. As figuras que seguem abaixo mostram os elementos que definimos acima:

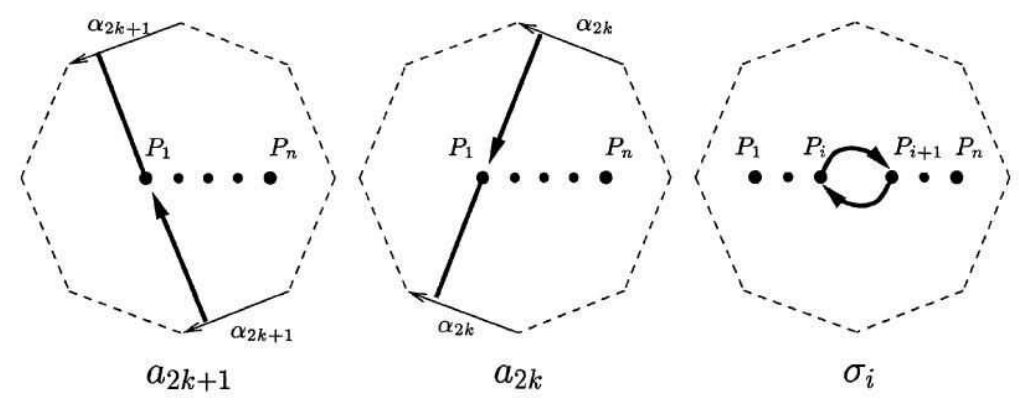

Figura 5.3: Elementos do grupo $B_{n}(M)$.

Vamos mostrar mais tarde que o conjunto $\left\{a_{1}, \ldots, a_{2 g}, \sigma_{1}, \ldots, \sigma_{n-1}\right\}$ gera o grupo $B_{n}(M)$. Pode-se provar (ver $[\overline{\mathrm{PR}}]$ ) que existe uma injeção do grupo $B(n)$ das tranças no disco no grupo $B_{n}(M)$ das tranças em $M$, sendo $M$ diferente da esfera e do plano projetivo. Logo, as relações clássicas de $B(n)$ :

$$
\begin{aligned}
\sigma_{i} \sigma_{j} & =\sigma_{j} \sigma_{i}, \text { se }|i-j| \geq 2 \\
\sigma_{i} \sigma_{i+1} \sigma_{i} & =\sigma_{i+1} \sigma_{i} \sigma_{i+1}, \text { se } 1 \leq i \leq n-2
\end{aligned}
$$


são válidas em $B_{n}(M)$.

Vamos observar também que se $i \in\{2, \ldots, n-1\}$ e $r \in\{1, \ldots, 2 g\}$, então as cordas não triviais de $\sigma_{i}$ e a corda não trivial de $a_{r}$ podem ser tomadas disjuntas. Isso mostra que estas duas tranças comutam como mostra a figura abaixo:

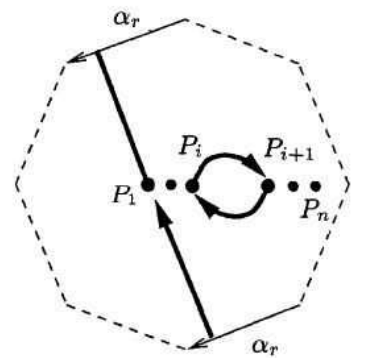

Figura 5.4: A trança $a_{r} \sigma_{i}$.

Assim, temos:

$$
a_{r} \sigma_{i}=\sigma_{i} a_{r}, \text { se } 1 \leq r \leq 2 g ; i \geq 2
$$

Note que a relação acima foi extraída através do $P_{1}$-polígono. Poderíamos extraí-la também (mas talvez com mais dificuldade) se considerássemos a trança $a_{r} \sigma_{i}$ no cilindro do polígono inicial:

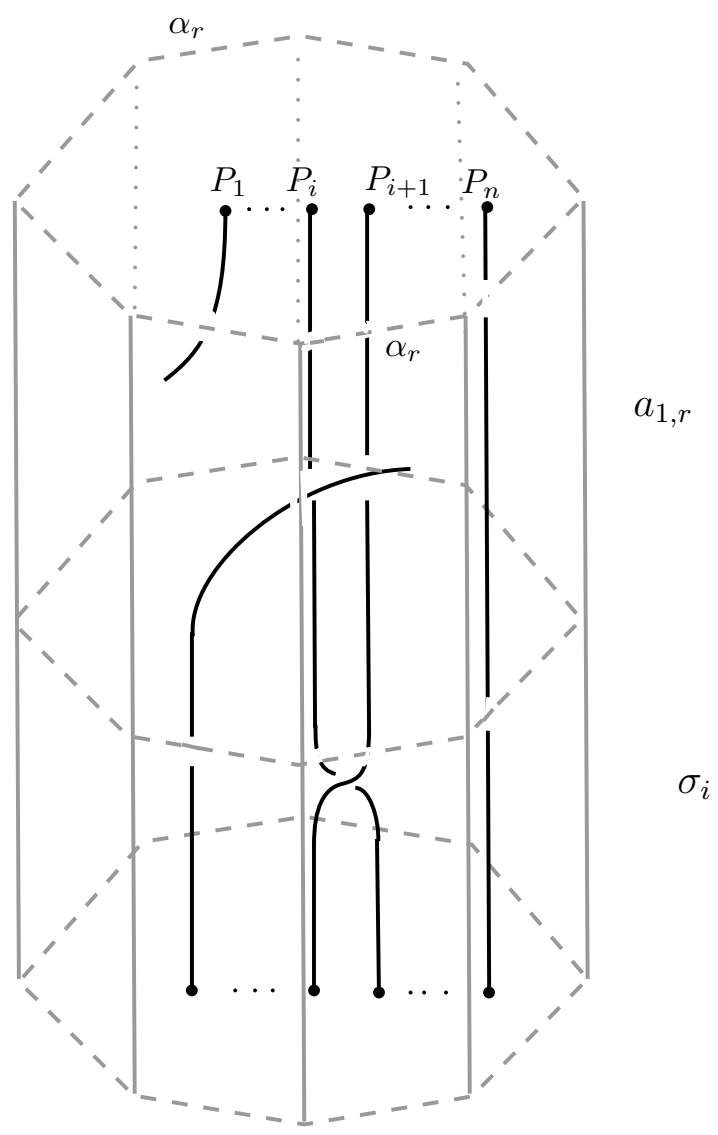


Agora, para encontrar mais relações para o conjunto de geradores, faremos a seguinte contrução: vamos denotar por $s_{r}$ a primeira corda de $a_{r}$, para todo $r=1, \ldots, 2 g$, e consideremos todos os caminhos $s_{1}, \ldots, s_{2 g}$. Podemos "cortar" o polígono $L$ ao longo desses caminhos e "colar" os pedaços ao longo dos caminhos $\alpha_{1}, \ldots, \alpha_{2 g}$. Desta forma, obtemos outro polígono de $4 g$ lados os quais são rotulados por $s_{1}, \ldots, s_{2 g}$. Vamos chamar este novo polígono de $P_{1}$-polígono de $M$ já que todos os seus vértices são identificados em $P_{1}$, enquanto $L$ será chamado de polígono inicial. Desta maneira, obtemos uma nova representação da superfície $M$ :
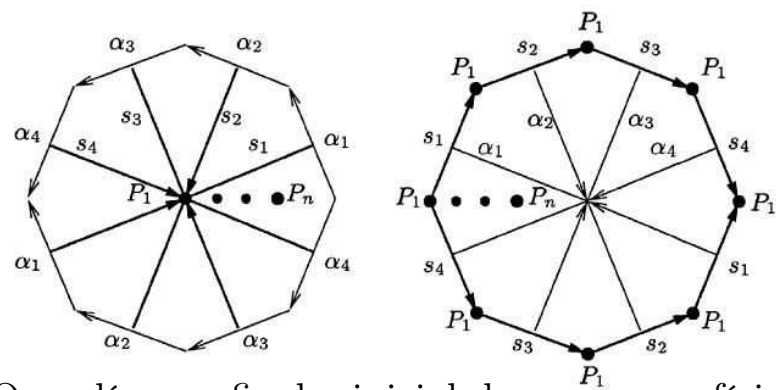

Figura 5.5: Os polígonos final e inicial de uma superfície de gênero 2.

O objetivo de construirmos o $P_{1}$-polígono é usá-lo para mostrar mais três relações em $B_{n}(M)$. Por exemplo, consideremos a trança $a_{1} \ldots a_{2 g} a_{1}^{-1} \ldots a_{2 g}^{-1}$. Se olharmos no $P_{1^{-}}$ polígono, veremos que a trança mencionada acima é equivalente a figura abaixo:

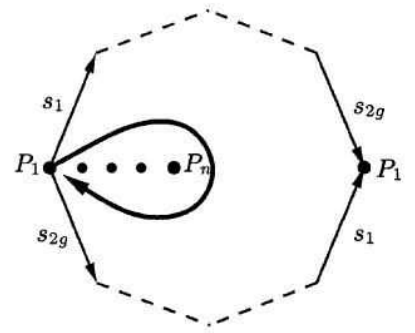

Figura 5.6: A trança $a_{1} \ldots a_{2 g} a_{1}^{-1} \ldots a_{2 g}^{-1}$

Mas vamos observar que a trança da figura acima pode ser vista no polígono inicial como uma trança que não atravessa paredes, ou seja, um elemento de $B(n)$, o grupo de tranças do disco. Logo, é fácil ver que esta trança é equivalente à trança $\sigma_{1} \ldots \sigma_{n-2} \sigma_{n-1}^{2} \sigma_{n-2} \ldots \sigma_{1}$.

Desta forma, obtemos a seguinte relação:

$$
a_{1} \ldots a_{2 g} a_{1}^{-1} \ldots a_{2 g}^{-1}=\sigma_{1} \ldots \sigma_{n-2} \sigma_{n-1}^{2} \sigma_{n-2} \ldots \sigma_{1} .
$$

Como observamos anteriormente podemos ver a trança $a_{1} \ldots a_{2 g} a_{1}^{-1} \ldots a_{2 g}^{-1}$ no cilindro do 
polígono inicial:

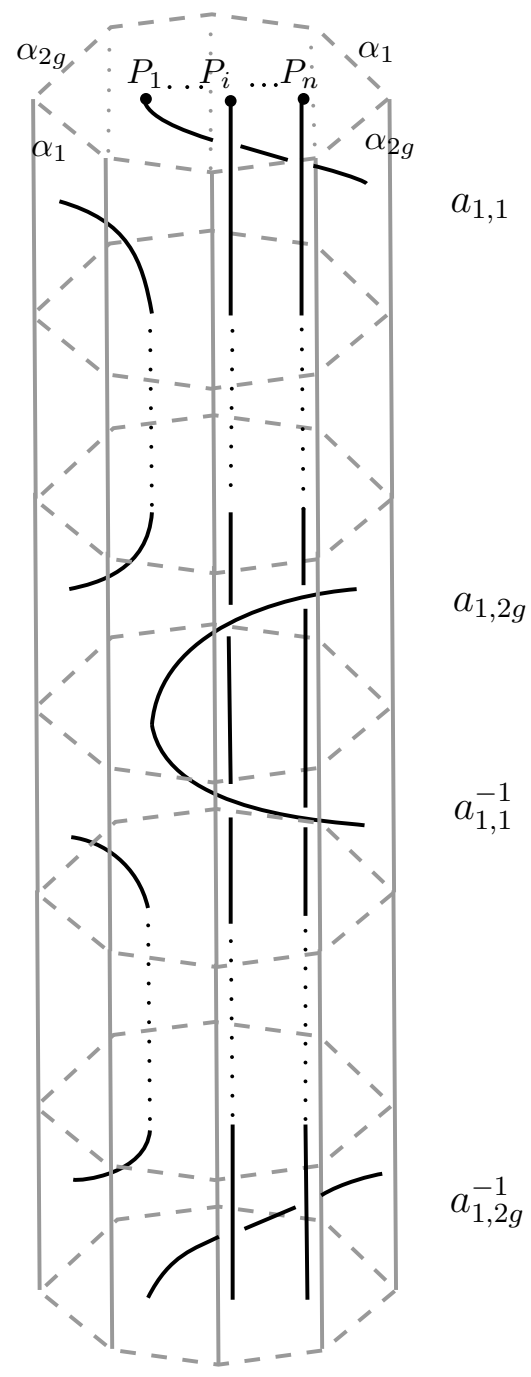

Agora, vamos definir para cada $r=1, \ldots, 2 g$ a trança:

$$
A_{2, r}=\sigma_{1}^{-1}\left(a_{1} \ldots a_{r-1} a_{r+1}^{-1} \ldots a_{2 g}^{-1}\right) \sigma_{1}^{-1}
$$
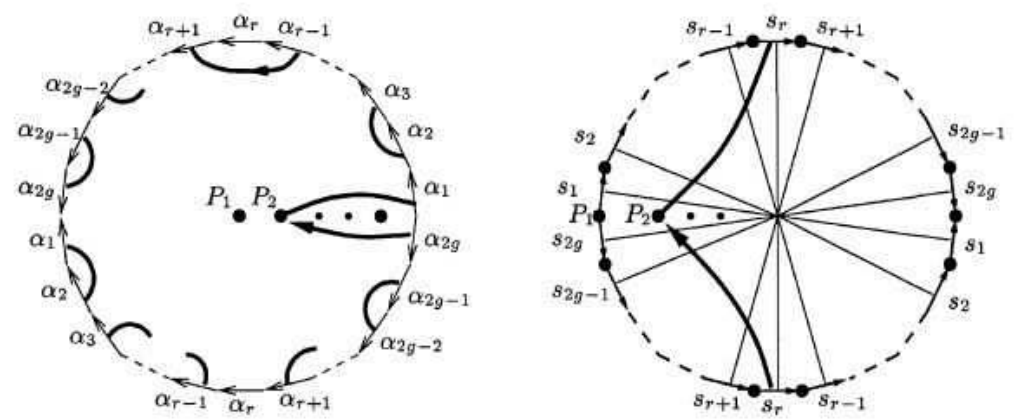

Figura 5.7: A trança $A_{2, r}$ no polígono inicial e no $P_{1}$-polígono 
No lado esquerdo da Figura 5.7, podemos ver uma trança a qual é equivalente a $A_{2, r}$ (se $r$ for ímpar, o caso par é análogo). Se "cortarmos" e "colarmos" da forma definida anteriormente para vermos esta trança no $P_{1}$-polígono, obteremos a situação representada pelo lado direito da Figura 5.7, ou seja, $A_{2, r}$ pode ser vista como uma trança cuja a única corda não trivial é a segunda, a qual sai do ponto $P_{2}$ "para cima" e atravessa a $r$-ésima parede $s_{r}$. Notemos que, ao contrário de $a_{r}, A_{2, r}$ sempre vai "para cima" no $P_{1^{-}}$ polígono, não importando a paridade de $r$. Desta forma, vemos que a trança $A_{2, r}$ pode ser representada por uma trança geométrica, na qual a única corda não trivial pode pode ser tomada disjunta de todos os caminhos $s_{t}$, com $t \neq r$. Logo, temos:

$$
a_{t} A_{2, r}=A_{2, r} a_{t}, \text { se } 1 \leq t, r \leq 2 g ; t \neq r .
$$

Vamos terminar nosso conjunto de relações considerando o comutador das tranças $\left(a_{1} \ldots a_{r}\right)$ e $A_{2, r}$, para todo $r=1, \ldots, 2 g$

A figura a seguir é um esboço da homotopia que começa com o comutador e deforma-o numa trança equivalente a $\sigma_{1}^{2}$ :
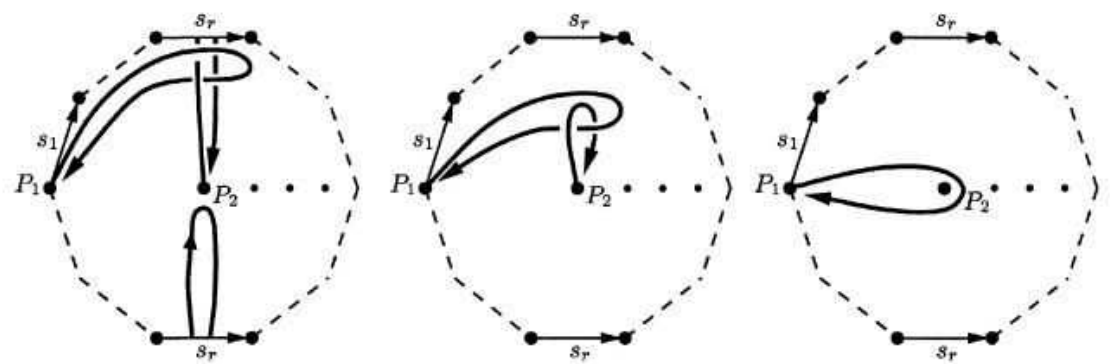

Figura 5.8: A trança $\left[a_{1} \ldots a_{r}, A_{2, r}\right]$

Desta forma, obtemos a seguinte relação:

$$
\left(a_{1} \ldots a_{r}\right) A_{2, r}=\sigma_{1}^{2} A_{2, r}\left(a_{1} \ldots a_{r}\right), \text { se } 1 \leq r \leq 2 g .
$$

Veremos mais tarde que as relações que acabamos de verificar formam um conjunto completo de relações definidoras de $B_{n}(M)$. Em outras palavras, temos o seguinte resultado que é um dos propósitos deste artigo:

Teorema 5.2.2. Se $M$ for uma superfície fechada, orientável de gênero $g \geq 1$, então $B_{n}(M)$ admitirá a seguinte apresentação:

- Geradores: $\sigma_{1}, \ldots, \sigma_{n-1}, a_{1}, \ldots, a_{2 g}$ 
- Relações:

(R1) $\sigma_{i} \sigma_{j}=\sigma_{j} \sigma_{i}$

(R2) $\sigma_{i} \sigma_{i+1} \sigma_{i}=\sigma_{i+1} \sigma_{i} \sigma_{i+1}$

(R3) $a_{1} \ldots a_{2 g} a_{1}^{-1} \ldots a_{2 g}^{-1}=\sigma_{1} \ldots \sigma_{n-2} \sigma_{n-1}^{2} \sigma_{n-2} \ldots \sigma_{1}$

(R4) $a_{r} A_{2, s}=A_{2, s} a_{r}$

(R5) $\left(a_{1} \ldots a_{r}\right) A_{2, r}=\sigma_{1}^{2} A_{2, r}\left(a_{1} \ldots a_{r}\right)$

(R6) $a_{r} \sigma_{i}=\sigma_{i} a_{r}$

$$
|i-j| \geq 2
$$$$
1 \leq i \leq n-2
$$

$1 \leq r, s \leq 2 g ; r \neq s$

$$
1 \leq r \leq 2 g
$$

$1 \leq r \leq 2 g ; i \geq 2$

onde:

$$
A_{2, r}=\sigma_{1}^{-1}\left(a_{1} \ldots a_{r-1} a_{r+1}^{-1} \ldots a_{2 g}^{-1}\right) \sigma_{1}^{-1}
$$

\subsubsection{Caso não-orientável}

Vamos considerar $M$ uma superfície fechada, não-orientável, com gênero $g \geq 2$, portanto diferente do plano projetivo. Para representar uma trança em $M$ também faremos uso de um polígono, mas desta vez, de $2 g+2$ lados como mostra a figura abaixo:

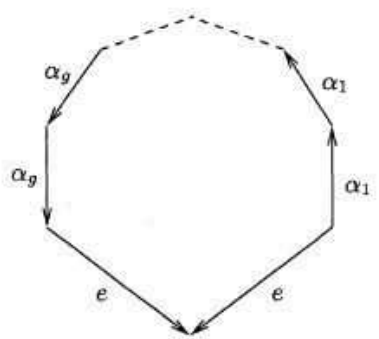

Figura 5.9: O polígono que representa a superfície $M$.

Observemos que os dois lados rotulados por $e$ são irrelevantes, mas eles serão importantes para definirmos os geradores de forma mais fácil.

Os geradores de $B_{n}(M)$ serão similares aos geradores do grupo das tranças em superfícies orientáveis. Para todo $i \in\{1, \ldots, n-1\}$, a trança $\sigma_{i}$ será a mesma do caso orientável. Agora, para todo $r \in\{1, \ldots, g\}$, a trança $a_{r}$ consiste em passar a primeira corda através da $r$-ésima parede, enquanto as outras cordas são caminhos triviais como mostra a Figura 5.10: 

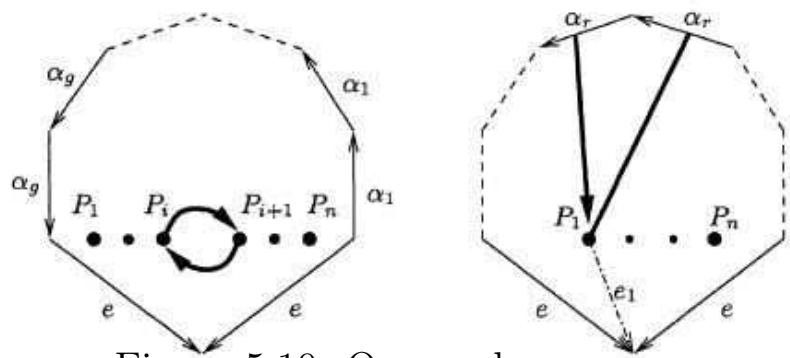

Figura 5.10: Os geradores $\sigma_{i}$ e $a_{r}$.

Notemos que ainda na Figura 5.10 definimos um caminho $e_{1}$ que liga $P_{1}$ ao ponto final de $e$. Este caminho é definido com o objetivo de conseguirmos seguir o mesmo procedimento do caso orientável na definição do $P_{1^{-}}$polígono de $M$ : vamos denotar por $s_{1}, \ldots, s_{g}$ a primeira corda de $a_{1}, \ldots, a_{g}$ respectivamente. Se "cortarmos" o polígono ao longo dos caminhos $s_{1}, \ldots, s_{g}, e_{1}$ e "colarmos" ao longo de $\alpha_{1}, \ldots, \alpha_{g}$, e então o resultado será o $P_{1}$-polígono de $M$ cujos lados, no sentido horário, são rotulados por $s_{1}, s_{1}, s_{2}, s_{2}, \ldots, s_{g}, s_{g}, e_{1}, e_{1}^{-1}$. Vamos definir a trança:

$$
A_{2, r}=\sigma_{1}^{-1}\left(a_{1}^{2} \ldots a_{r-1}^{2} a_{r}^{-1} a_{r-1}^{-2} \ldots a_{1}^{-2}\right) \sigma_{1},
$$

que é representada através do $P_{1}$-polígono indicado na figura a seguir:

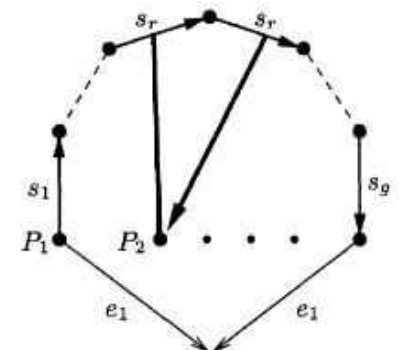

Figura 5.11: $\mathrm{O} P_{1}$-polígono de $A_{2, r}$.

Agora, da mesma forma que fizemos na seção anterior, vamos mostrar que existem seis relações para $B_{n}(M)$ que são análogas as do caso orientável. Elas são encontradas da mesma forma que encontramos as relações da seção anterior mas agora utilizamos o $P_{1}$-polígono correspondente ao caso não orientável.

Observemos ainda na Figura 5.11 que $A_{2, r}$ pode ser vista como uma trança cuja a única corda não trivial é a segunda, que sai do ponto $P_{2}$ "para cima" e atravessa a $r$-ésima parede $s_{r}$. E desta forma oservamos que:

$$
a_{t} A_{2, r}=A_{2, r} a_{t}, 1 \leq t, r \leq g ; t \neq r .
$$

As relações do grupo de tranças no disco $B(n)$ são válidas pela mesma injeção men- 
cionada no caso orientável. Consideremos agora a trança $a_{1}^{2} \ldots a_{g}^{2}$. Construindo seu $P_{1}$ polígono, temos:

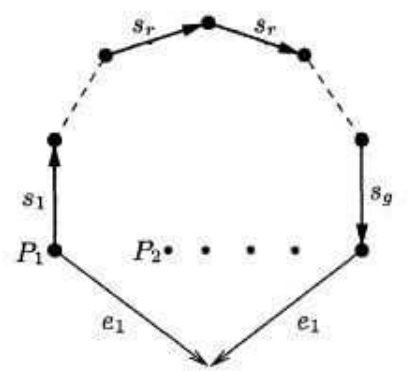

Figura 5.12: O $P_{1}$-polígono de $a_{1}^{2} \ldots a_{g}^{2}$.

Claramente, esta trança é equivalente a $\sigma_{1} \ldots \sigma_{n-2} \sigma_{n-1}^{2} \sigma_{n-2} \ldots \sigma_{1}$. Portanto,

$$
a_{1}^{2} \ldots a_{g}^{2}=\sigma_{1} \ldots \sigma_{n-2} \sigma_{n-1}^{2} \sigma_{n-2} \ldots \sigma_{1}
$$

Da mesma forma que fizemos no caso orientável, a trança $a_{1}^{2} \ldots a_{g}^{2}$ vista no cilindro do polígono inicial é dada na figura que segue:

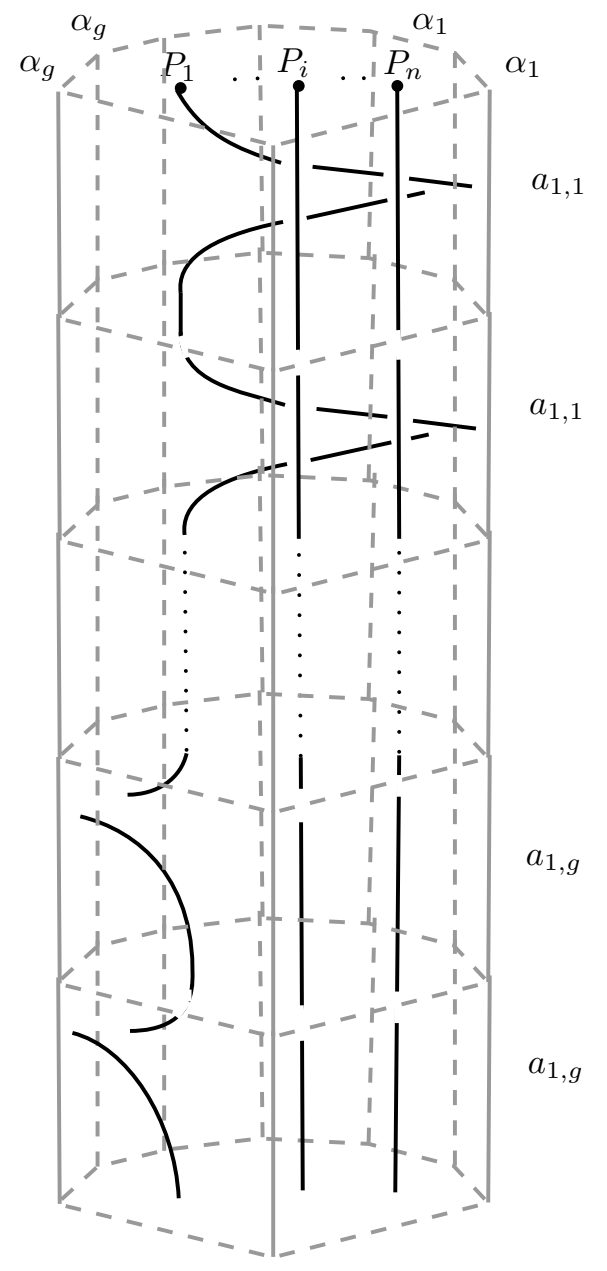


Agora, restam duas relações a serem exibidas. De forma análoga ao caso orientável, temos que se $j \in\{2, \ldots, n-1\}$ e $r \in\{1, \ldots, g\}$ então $a_{r} \sigma_{j}=\sigma_{j} a_{r}, 1 \leq r \leq g ; j \geq 2$ desde que estas duas tranças podem ser tomadas disjuntas como mostra a figura a seguir:

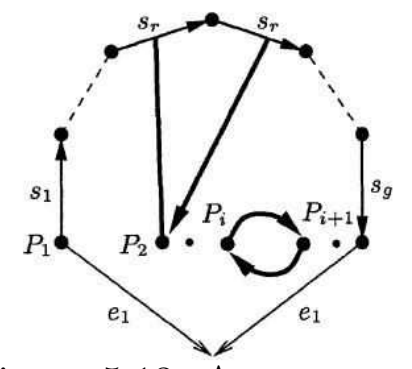

Figura 5.13: A trança $a_{r} \sigma_{j}$.

E, esta trança no cilindro do polígono inicial é representada na figura abaixo:

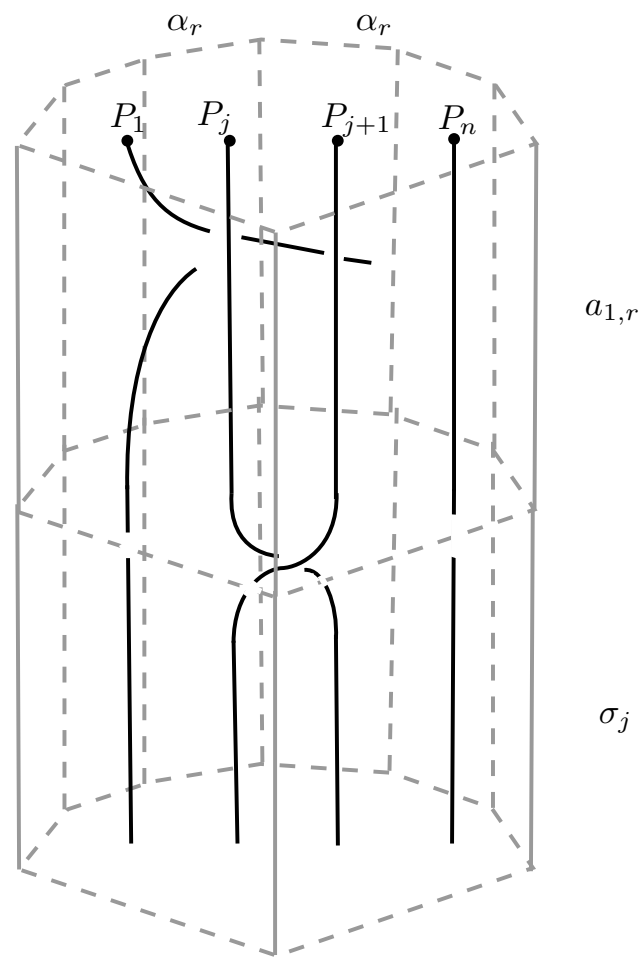

E, finalmente, podemos ver a relação do comutador das tranças $\left(a_{1}^{2} \ldots a_{r-1}^{2} a_{r}\right)$ e $A_{2, r}$ :

$$
\left(a_{1}^{2} \ldots a_{r-1}^{2} a_{r}\right) A_{2, r}=\sigma_{1}^{2} A_{2, r}\left(a_{1}^{2} \ldots a_{r-1}^{2} a_{r}\right), \text { se } 1 \leq r \leq g .
$$

que justificamos pela representação abaixo: 

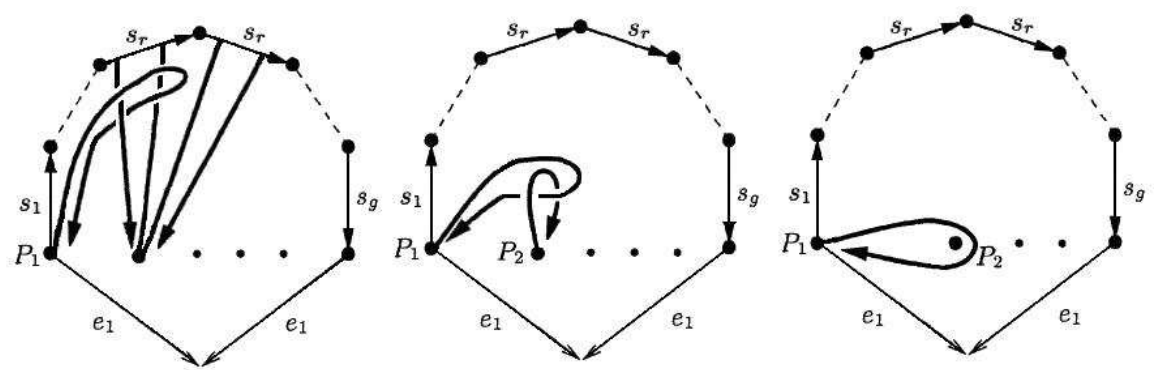

Figura 5.14: A trança $\left[a_{1}^{2} \ldots a_{r-1}^{2} a_{r}, A_{2, r}\right]$

Assim, a partir das considerações feitas, provaremos posteriormente o seguinte resultado:

Teorema 5.2.3. Se $M$ for uma superfície fechada, não orientável de gênero $g \geq 2$, então $B_{n}(M)$ admitirá a seguinte apresentação:

- Geradores: $\sigma_{1}, \ldots, \sigma_{n-1}, a_{1}, \ldots, a_{g}$

- Relações:

$$
\begin{array}{lr}
\text { (r1) } \sigma_{i} \sigma_{j}=\sigma_{j} \sigma_{i} & |i-j| \geq 2 \\
\text { (r2) } \sigma_{i} \sigma_{i+1} \sigma_{i}=\sigma_{i+1} \sigma_{i} \sigma_{i+1} & 1 \leq i \leq n-2 \\
\text { (r3) } a_{1}^{2} \ldots a_{g}^{2}=\sigma_{1} \ldots \sigma_{n-2} \sigma_{n-1}^{2} \sigma_{n-2} \ldots \sigma_{1} & \\
\text { (r4) } a_{r} A_{2, s}=A_{2, s} a_{r} & 1 \leq r, s \leq g ; r \neq s \\
\text { (r5) }\left(a_{1}^{2} \ldots a_{r-1}^{2} a_{r}\right) A_{2, r}=\sigma_{1}^{2} A_{2, r}\left(a_{1}^{2} \ldots a_{r-1}^{2} a_{r}\right) & 1 \leq r \leq g \\
\text { (r6) } a_{r} \sigma_{i}=\sigma_{i} a_{r} & 1 \leq r \leq g ; i \geq 2
\end{array}
$$

onde:

$$
A_{2, r}=\sigma_{1}^{-1}\left(a_{1}^{2} \ldots a_{r-1}^{2} a_{r}^{-1} a_{r-1}^{-2} \ldots a_{1}^{-2}\right) \sigma_{1} .
$$

\subsection{As ideias chave das demonstrações}

Nesta seção vamos dar a ideia de como provaremos os teoremas enunciados nas duas seções anteriores deste capítulo. Mas, antes disso, vamos relembrar um teorema muito 
importante visto no Capítulo 2 deste trabalho que será muito usado nas demonstrações dos teoremas em questão.

Vamos considerar uma sequência exata de grupos e homomorfismos:

$$
1 \longrightarrow A \stackrel{i}{\longrightarrow} B \stackrel{p}{\longrightarrow} C \longrightarrow 1
$$

onde estaremos supondo que $A \subset B$ e $i$ é o homomorfismo inclusão. Suponhamos também que $A$ e $C$ tenham as apresentações $<G_{A} ; R_{A}>$ e $<G_{C} ; R_{C}>$ respectivamente.

Para cada $y \in G_{C}$, escolhemos um elemento $\tilde{y} \in B$ tal que $p(\tilde{y})=y$, e, para cada relator $r=y_{1} \ldots y_{m} \in R_{C}$, escrevemos $\tilde{r}=\tilde{y}_{1} \ldots \tilde{y}_{m} \in B$. Então, para cada $r \in R_{C}$, existe uma palavra $f_{r}$ em $G_{A}$, não necessariamente única, tal que $\tilde{r}=f_{r}$ em $B$. Por outro lado, para cada $x \in G_{A}$ e $y \in G_{C}$, existe uma palavra $g_{x, y}$ em $G_{A}$, não necessariamente única, tal que $\tilde{y} x \tilde{y}^{-1}=g_{x, y}$ em $B$. Escolhemos então palavras $f_{r}$ e $g_{x, y}$ como acima.

Teorema 5.3.1. Sob as condições acima, $B$ adimitirá a seguinte apresentação:

Geradores: $\left\{G_{A}\right\} \cup\left\{\tilde{y} ; y \in G_{C}\right\}$

Relações:

- Tipo 1: $r_{A}=1$, para cada $r_{A} \in R_{A}$.

- Tipo 2: $\tilde{r}=f_{r}$, para cada $r \in R_{C}$.

- Tipo 3: $\tilde{y} x \tilde{y}^{-1}=g_{x, y}$, para cada $x \in G_{A}$ e cada $y \in G_{C}$.

O propósito de termos enunciado novamente este resultado também foi reescrevê-lo com notações que tornarão a demonstração dos teoremas mais simples.

E, finalmente, a idéia de como faremos as demonstrações será dada por meio de 3 passos:

Passo 1: Vamos introduzir um grupo abstrato $\overline{P B_{n}(M)}$, dado por sua apresentação e definir um homomorfismo

$$
\overline{P B_{n}(M)} \stackrel{\varphi}{\longrightarrow} P B_{n}(M) \text {. }
$$


Passo 2: Provaremos por indução sobre $n$ que $\varphi$ é um isomorfismo, aplicando o Teorema 5.3.1 para a sequência exata (2):

$$
1 \longrightarrow \pi_{1}\left(M \backslash \mathcal{P}^{\prime}, P_{1}\right) \stackrel{u}{\longrightarrow} P B_{n}(M, \mathcal{P}) \stackrel{\nu}{\longrightarrow} P B_{n-1}\left(M, \mathcal{P}^{\prime}\right) \longrightarrow 1
$$

Passo 3: Denotaremos por $\overline{B_{n}(M)}$ o grupo abstrato dado pela apresentação do Teorema 5.2.2 se $M$ for orientável e dado pela apresentação do Teorema 5.2.3 se $M$ for não orientável. Vamos mostrar que existe um homomorfismo bem definido

$$
\overline{B_{n}(M)} \stackrel{\psi}{\longrightarrow} B_{n}(M)
$$

e, aplicando o Teorema 5.3.1 na sequência exata (1):

$$
1 \longrightarrow P B_{n}(M) \stackrel{e}{\longrightarrow} B_{n}(M) \stackrel{f}{\longrightarrow} \Sigma_{n} \longrightarrow 1
$$

mostraremos que $\psi$ é um isomorfismo.

Observação 5.3.2. No passo 2 da demonstração tanto no caso orientável como no caso não orientável, vamos extrair mais dois resultados interessantes que são também objetivos deste trabalho: uma apresentação para $P B_{n}(M)$ quando $M$ for orientável e uma apresentação para $P B_{n}(M)$ quando $M$ for não orientável.

\subsection{O grupo de tranças de uma superfície orientável}

Nesta seção provaremos o Teorema 5.2.2 segundo o procedimento dado na seção anterior. Então aqui, assumiremos $M$ uma superfície fechada, orientável de gênero $g \geq 1$.

Passo 1. Vamos definir o grupo abstrato $\overline{P B_{n}(M)}$ que admite a seguinte apresentação: Apresentação 1:

Geradores: $\left\{a_{i, r} ; 1 \leq i \leq n, 1 \leq r \leq 2 g\right\} \cup\left\{T_{j, k} ; 1 \leq j<k \leq n\right\}$.

\section{Relações:}

(PR1) $a_{n, 1}^{-1} a_{n, 2}^{-1} \ldots a_{n, 2 g}^{-1} a_{n, 1} a_{n, 2} \ldots a_{n, 2 g}=\prod_{i=1}^{n-1} T_{i, n-1}^{-1} T_{i, n}$. 


$$
\begin{aligned}
& \text { (PR2) } a_{i, r} A_{j, s}=A_{j, s} a_{i, r} \\
& 1 \leq i<j \leq n ; 1 \leq r, s \leq 2 g ; r \neq s . \\
& \text { (PR3) }\left(a_{i, 1} \ldots a_{i, r}\right) A_{j, r}\left(a_{i, r}^{-1} \ldots a_{i, 1}^{-1}\right) A_{j, r}^{-1}=T_{i, j} T_{i, j-1}^{-1} \\
& 1 \leq i<j \leq n ; 1 \leq r \leq 2 g \text {. } \\
& \text { (PR4) } T_{i, j} T_{k, l}=T_{k, l} T_{i, j} \\
& 1 \leq i<j<k<l \leq n \text { ou } 1 \leq i<k<l \leq j \leq n . \\
& \text { (PR5) } T_{k, l} T_{i, j} T_{k, l}^{-1}=T_{i, k-1} T_{i, k}^{-1} T_{i, j} T_{i, l}^{-1} T_{i, k} T_{i, k-1}^{-1} T_{i, l} \\
& 1 \leq i<k \leq j<l \leq n . \\
& \text { (PR6) } a_{i, r} T_{j, k}=T_{j, k} a_{i, r} \\
& 1 \leq i<j<k \leq n \text { ou } 1 \leq j<k<i \leq n ; 1 \leq r \leq 2 g . \\
& a_{i, r}\left(a_{j, 2 g}^{-1} \ldots a_{j, 1}^{-1} T_{j, k} a_{j, 2 g} \ldots a_{j, 1}\right)=\left(a_{j, 2 g}^{-1} \ldots a_{j, 1}^{-1} T_{j, k} a_{j, 2 g} \ldots a_{j, 1}\right) a_{i, r} \quad 1 \leq j<i \leq k \leq n . \\
& T_{j, n}=\left(\prod_{i=1}^{j-1} a_{i, 2 g}^{-1} \ldots a_{i, 1}^{-1} T_{i, j-1} T_{i, j}^{-1} a_{i, 1} \ldots a_{i, 2 g}\right) a_{j, 1} \ldots a_{j, 2 g} a_{j, 1}^{-1} \ldots a_{j, 2 g}^{-1}
\end{aligned}
$$

Onde

$$
A_{j, s}=a_{j, 1} \ldots a_{j, s-1} a_{j, s+1}^{-1} \ldots a_{j, 2 g}^{-1}
$$

Agora, vamos enunciar um lema que nos garantirá outra apresentação de $\overline{P B_{n}(M)}$ :

Lema 5.4.1. Sejam F um grupo livre livremente gerado por $\left\{x_{1}, \ldots, x_{2 g}\right\}$. Seja também $X_{r}=x_{1} \ldots x_{r-1} x_{r+1}^{-1} \ldots x_{2 g}^{-1}$. Então $\left\{X_{1}, \ldots, X_{2 g}\right\}$ é um sistema livre de geradores de $F$.

Demonstração: A demonstração é bem simples e a fórmula da mudança de geradores é dada por:

$$
\begin{aligned}
x_{k} & =\left(X_{1} X_{2}^{-1} \ldots X_{k-2} X_{k-1}^{-1}\right)\left(X_{k+1} X_{k+2}^{-1} \ldots X_{2 g-1}^{-1} X_{2 g}\right), \\
x_{k}^{-1} & =\left(X_{1} X_{2}^{-1} \ldots X_{k-2}^{-1} X_{k-1}\right)\left(X_{k+1}^{-1} X_{k+2} \ldots X_{2 g-1}^{-1} X_{2 g}\right),
\end{aligned}
$$

Aplicando o lema anterior para o conjunto de geradores $\left\{a_{i, r} ; 1 \leq r \leq 2 g\right\}$ de $\overline{P B_{n}(M)}$ $\operatorname{com} A_{j, r}=a_{j, 1} \ldots a_{j, r-1} a_{j, r+1}^{-1} \ldots a_{j, 2 g}^{-1}$ teremos:

\section{Apresentação 2:}

Geradores: $\left\{A_{i, r} ; 1 \leq i \leq n, 1 \leq r \leq 2 g\right\} \cup\left\{T_{j, k} ; 1 \leq j<k \leq n\right\}$.

Relações: as mesmas da apresentação 1, onde:

$$
\begin{aligned}
& a_{i, k}=\left(A_{i, 1} A_{i, 2}^{-1} \ldots A_{i, k-2} A_{i, k-1}^{-1}\right)\left(A_{i, k+1} A_{i, k+2}^{-1} \ldots A_{i, 2 g-1}^{-1} A_{i, 2 g}\right) \quad \text { se k for ímpar, } \\
& a_{i, k}^{-1}=\left(A_{i, 1} A_{i, 2}^{-1} \ldots A_{i, k-2}^{-1} A_{i, k-1}\right)\left(A_{i, k+1}^{-1} A_{i, k+2} \ldots A_{i, 2 g-1}^{-1} A_{i, 2 g}\right) \quad \text { se k for par. }
\end{aligned}
$$

Consideremos agora a apresentação 1 de $\overline{P B_{n}(M)}$. Vamos definir o homomorfismo $\varphi$ entre $\overline{P B_{n}(M)}$ e $P B_{n}(M)$ dado por: 


$$
\begin{aligned}
\varphi & : \overline{P B_{n}(M)} \rightarrow P B_{n}(M) \\
a_{i, r} \longmapsto a_{i, r}, & 1 \leq i \leq n, 1 \leq r \leq 2 g \\
& T_{i, j} \longmapsto T_{i, j}, \quad 1 \leq i<j \leq n .
\end{aligned}
$$

Por abuso de notação, continuaremos denotando por $a_{i, r}$ e $T_{i, j}$ as tranças que serão as imagens de $a_{i, r}$ e $T_{i, j}$ respectivamente sobre o homomorfismo $\varphi$. Essas tranças são definidas como segue:

- Em $a_{i, r}$, a $i$-ésima corda sai do ponto $P_{i}$ e passa pela $r$-ésima parede. Esta corda vai "para cima" se $r$ for ímpar, e "para baixo" caso contrário. As outras cordas são triviais. Vamos observar que $a_{1, r}=a_{r}$, para cada $r$.

- Em $T_{i, j}$ a $i$-ésima corda passa em torno dos pontos $P_{i+1}, \ldots, P_{j}$ como na Figura 5.15, enquanto as outras cordas são triviais. Se $i=j$, vamos definir $T_{i, j}$ como sendo a trança trivial.

As tranças definidas acima podem ser vistas na figura abaixo:

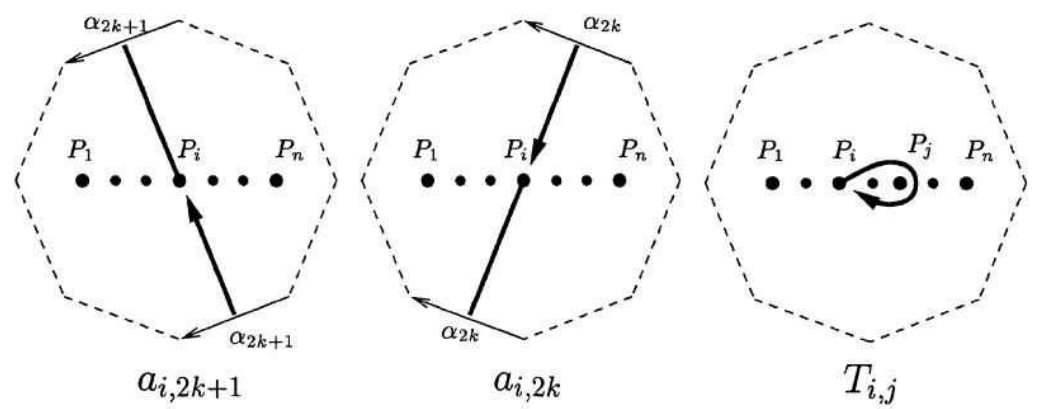

Figura 5.15: Os geradores de $P B_{n}(M)$.

Vamos denotar por $s_{i, r}$ a $i$-ésima corda de $a_{i, r}$, e por $t_{i, j}$ a $i$-ésima corda de $T_{i, j}$. É fácil vermos que para cada $i$, o conjunto de caminhos $\left\{s_{i, 1}, \ldots, s_{i, 2 g}\right\}$ gera $\pi_{1}(M)$.

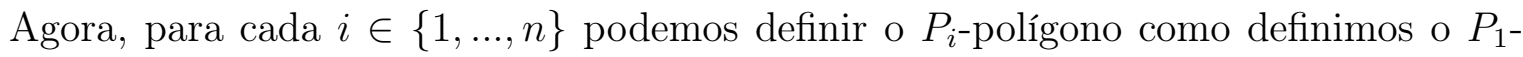
polígono: nós "cortamos" $L$ ao longo de $s_{i, 1}, \ldots, s_{i, 2 g}$ e "colamos" ao longo de $\alpha_{1}, \ldots, \alpha_{2 g}$. Vamos definir também, para cada $2 \leq j \leq n$ e $1 \leq r \leq 2 g$, a trança:

$$
A_{j, r}=a_{j, 1} \ldots a_{j, r-1} a_{j, r+1}^{-1} \ldots a_{j, 2 g}^{-1} .
$$


Como na representação de $A_{2, r}$ no $P_{1}$-polígono considerado na seção $5.2, A_{j, r}$ pode ser representado no $P_{i}$-polígono (para $1 \leq i<j$ ), como a trança da figura a seguir, na qual a única corda não trivial é a $j$-ésima, que vai "para cima" e cruza uma vez a $r$-ésima parede $s_{i, r}$. Notemos que esta representação não depende de $i$, mas é válida somente quando $i<j$.

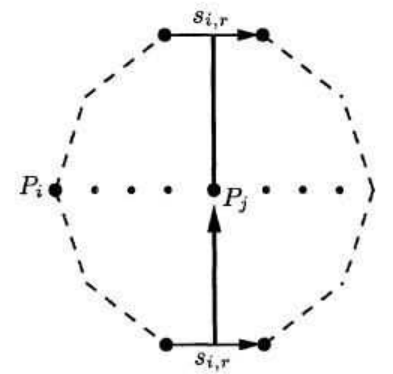

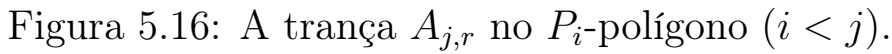

Agora, definimos $\varphi$ da forma mais natural possível. Para mostrar que $\varphi$ é um homomorfismo, temos que mostrar que as relações de $\overline{P B_{n}(M)}$ são válidas em $P B_{n}(M)$. De fato:

- As relações $(P R 4)$ e $(P R 5)$ valem em $P B_{n}(M)$ já que estas tranças podem ser vistas como tranças puras no disco. A representação que deixa evidente que a relação $(P R 4)$ é válida em $P B_{n}(M)$ segue abaixo:

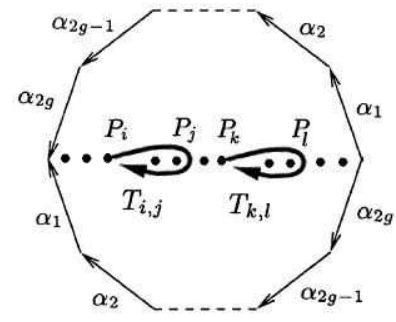

Figura 5.17: $T_{i, j} T_{k, l}=T_{k, l} T_{i, j}, 1 \leq i<j<k<l \leq n$.

Para a relação $(P R 5)$ também é simples: isolamos todos os termos desta relação e mostramos que este produto é equivalente à trança trivial. Ela é válida em $P B_{n}(M)$ já que podemos vê-la como uma trança pura no disco.

- A relação (PR6) também é óbvia graças à representação seguinte: 


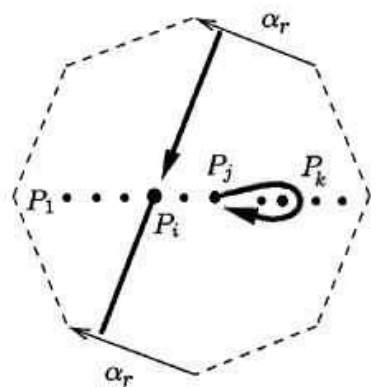

Figura 5.18: A trança $a_{i, r} T_{j, k}$.

- As relações $(P R 1),(P R 2)$ e $(P R 3)$ podem ser provadas que são válidas em $P B_{n}(M)$ da mesma forma que mostramos as relações $(R 1),(R 2)$ e $(R 3)$ respectivamente. De fato, para a relação $(P R 1)$ temos que o produto de tranças $\prod_{i=1}^{n-1} T_{i, n-1}^{-1} T_{i, n}$ é equivalente, via $P_{n}$-polígono, à trança:

$$
a_{n, 1}^{-1} a_{n, 2}^{-1} \ldots a_{n, 2 g}^{-1} a_{n, 1} a_{n, 2} \ldots a_{n, 2 g}
$$

que por sua vez é equivalente a trança:

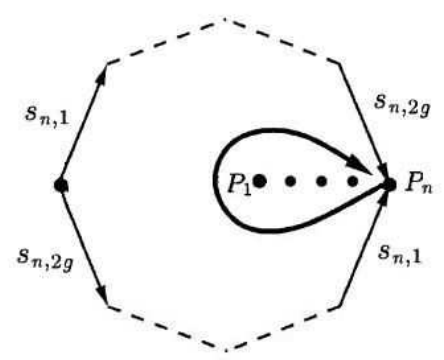

Figura 5.19: A trança $a_{n, 1}^{-1} a_{n, 2}^{-1} \ldots a_{n, 2 g}^{-1} a_{n, 1} a_{n, 2} \ldots a_{n, 2 g}$.

Portanto, a relação $(P R 1)$ é satisfeita em $P B_{n}(M)$. Agora, a validade da relação $(P R 2)$ em $P B_{n}(M)$ é clara com a ajuda da representação do $P_{i}$-polígono de $A_{j, s}$ $(i<j)$ da Figura 5.16.

Para a relação $(P R 3)$ também vamos construir o $P_{i}$-polígono da trança:

$$
\left(a_{i, 1} \ldots a_{i, r}\right) A_{j, r}\left(a_{i, r}^{-1} \ldots a_{i, 1}^{-1}\right) A_{j, r}^{-1}
$$

que é representada pela figura que segue: 


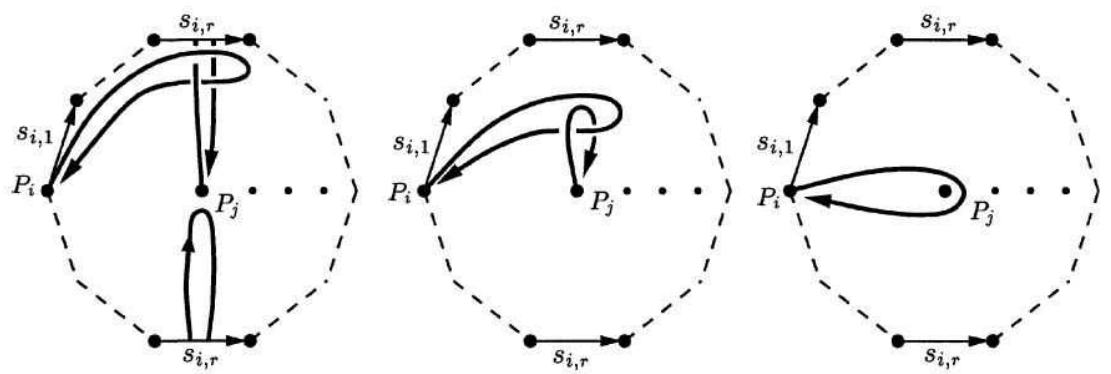

Figura 5.20: A trança $\left(a_{i, 1} \ldots a_{i, r}\right) A_{j, r}\left(a_{i, r}^{-1} \ldots a_{i, 1}^{-1}\right) A_{j, r}^{-1}$.

E observe que a última trança é equivalente à trança $T_{i, j} T_{i, j-1}^{-1}$. Portanto, a relação segue em $P B_{n}(M)$.

- Vamos considerar o $P_{j}$-polígono de $\left(a_{j, 2 g}^{-1} \ldots a_{j, 1}^{-1} T_{j, k} a_{j, 2 g} \ldots a_{j, 1}\right)$ para a verificação da relação $(P R 7)$ :

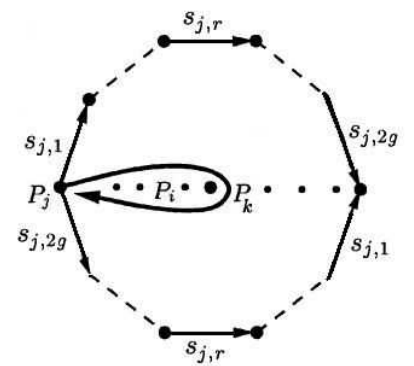

Figura 5.21: A trança $\left(a_{j, 2 g}^{-1} \ldots a_{j, 1}^{-1} T_{j, k} a_{j, 2 g} \ldots a_{j, 1}\right)$.

E assim, temos que $(P R 7)$ é válida em $P B_{n}(M)$.

- Finalmente, para verificarmos que a relação $(P R 8)$ é válida em $P B_{n}(M)$ vamos precisar de todos os $P_{i}$-polígonos, $i=1, \ldots, j-1$ da trança:

$$
a_{i, 2 g}^{-1} \ldots a_{i, 1}^{-1} T_{i, j-1} T_{i, j}^{-1} a_{i, 1} \ldots a_{i, 2 g}
$$

Se $i<j$ esta trança é equivalente à trança da figura a seguir:

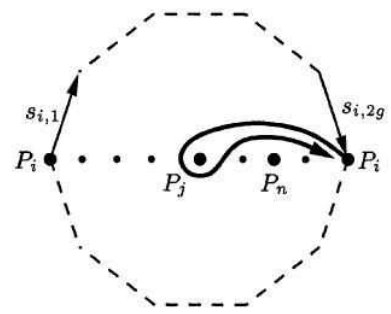

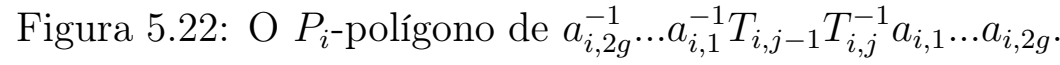


E a trança da figura acima é equivalente à trança representada pela figura abaixo no $P_{j}$-polígono:

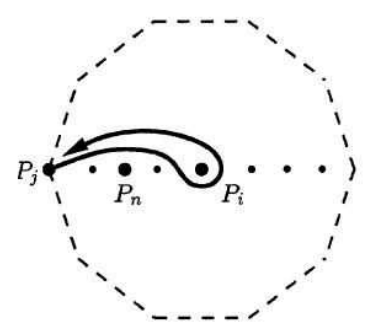

Figura 5.23: A trança $a_{i, 2 g}^{-1} \ldots a_{i, 1}^{-1} T_{i, j-1} T_{i, j}^{-1} a_{i, 1} \ldots a_{i, 2 g}$.

Assim, a trança $\prod_{i=1}^{j-1} a_{i, 2 g}^{-1} \ldots a_{i, 1}^{-1} T_{i, j-1} T_{i, j}^{-1} a_{i, 1} \ldots a_{i, 2 g}$ é equivalente à trança dada na figura que segue:

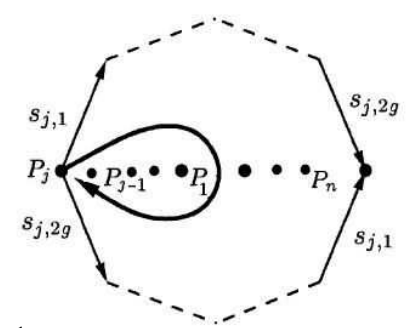

Figura 5.24: A trança $\left(\prod_{i=1}^{j-1} a_{i, 2 g}^{-1} \ldots a_{i, 1}^{-1} T_{i, j-1} T_{i, j}^{-1} a_{i, 1} \ldots a_{i, 2 g}\right) a_{j, 1} \ldots a_{j, 2 g} a_{j, 1}^{-1} \ldots a_{j, 2 g}^{-1}$.

que por sua vez é equivalente a $T_{j, n}$ e assim o resultado segue.

Pelas considerações anteriores mostramos que $\varphi$ é um homomorfismo, e assim terminamos o passo 1.

Passo 2. Vamos mostrar por indução sobre $n$ que $\varphi$ é um isomorfismo.

Consideremos:

$$
\begin{aligned}
\varphi: & \overline{P B_{n}(M)} \rightarrow P B_{n}(M) \\
& a_{i, r} \longmapsto a_{i, r}, 1 \leq i \leq n, 1 \leq r \leq 2 g \\
& T_{j, k} \longmapsto T_{j, k}, 1 \leq j<k \leq n .
\end{aligned}
$$

Se $n=1$ então a apresentação de $\overline{P B_{1}(M)}$ reduz-se à:

$$
\overline{P B_{1}(M)}=<\left\{a_{1,1}, \ldots, a_{1,2 g}\right\} ; a_{1,1}^{-1} \ldots a_{1,2 g}^{-1} a_{1,1} \ldots a_{1,2 g}=1>\text {. }
$$


Vamos observar que a apresentação acima é a mesma apresentação de $\pi_{1}(M)$ que estudamos no capítulo 3 e, na primeira seção deste capítulo vimos que $\pi_{1}(M)=P B_{1}(M)$. Mais ainda, desde que $n=1$ temos $\varphi\left(a_{1, r}\right)=a_{1, r}=s_{1, r}, r=1, \ldots, 2 g$. Logo, $\varphi$ é um homomorfismo de grupos que possuem as mesmas apresentações, o qual leva os geradores de $\overline{P B_{1}(M)}$ nos geradores de $P B_{1}(M)$. Portanto, $\overline{P B_{1}(M)} \stackrel{\varphi}{\simeq} P B_{1}(M)$.

Agora para a hipótese de indução suponhamos que $\overline{P B_{n-1}(M)} \stackrel{\varphi}{\simeq} P B_{n-1}(M)$. Logo, $P B_{n-1}(M)$ admite as apresentações 1 e 2 como apresentações:

\section{Apresentação 1:}

Geradores: $\left\{a_{i, r} ; 1 \leq i \leq n-1,1 \leq r \leq 2 g\right\} \cup\left\{T_{j, k} ; 1 \leq j<k \leq n-1\right\}$.

\section{Relações:}

(PR1) $a_{n-1,1}^{-1} a_{n-1,2}^{-1} \ldots a_{n-1,2 g}^{-1} a_{n-1,1} a_{n-1,2} \ldots a_{n-1,2 g}=\prod_{i=1}^{n-2} T_{i, n-2}^{-1} T_{i, n-1}$.

(PR2) $a_{i, r} A_{j, s}=A_{j, s} a_{i, r} \quad 1 \leq i<j \leq n-1 ; 1 \leq r, s \leq 2 g ; r \neq s$.

(PR3) $\left(a_{i, 1} \ldots a_{i, r}\right) A_{j, r}\left(a_{i, r}^{-1} \ldots a_{i, 1}^{-1}\right) A_{j, r}^{-1}=T_{i, j} T_{i, j-1}^{-1} \quad 1 \leq i<j \leq n-1 ; 1 \leq r \leq 2 g$.

(PR4) $T_{i, j} T_{k, l}=T_{k, l} T_{i, j} \quad 1 \leq i<j<k<l \leq n-1$ ou $1 \leq i<k<l \leq j \leq n-1$.

(PR5) $T_{k, l} T_{i, j} T_{k, l}^{-1}=T_{i, k-1} T_{i, k}^{-1} T_{i, j} T_{i, l}^{-1} T_{i, k} T_{i, k-1}^{-1} T_{i, l} \quad 1 \leq i<k \leq j<l \leq n-1$.

(PR6) $a_{i, r} T_{j, k}=T_{j, k} a_{i, r} \quad 1 \leq i<j<k \leq n-1$ ou $1 \leq j<k<i \leq n-1 ; 1 \leq r \leq 2 g$.

(PR7) $a_{i, r}\left(a_{j, 2 g}^{-1} \ldots a_{j, 1}^{-1} T_{j, k} a_{j, 2 g} \ldots a_{j, 1}\right)=\left(a_{j, 2 g}^{-1} \ldots a_{j, 1}^{-1} T_{j, k} a_{j, 2 g} \ldots a_{j, 1}\right) a_{i, r}$ $1 \leq j<i \leq k \leq n-1$.

(PR8) $T_{j, n-1}=\left(\prod_{i=1}^{j-1} a_{i, 2 g}^{-1} \ldots a_{i, 1}^{-1} T_{i, j-1} T_{i, j}^{-1} a_{i, 1} \ldots a_{i, 2 g}\right) a_{j, 1} \ldots a_{j, 2 g} a_{j, 1}^{-1} \ldots a_{j, 2 g}^{-1}$.

onde $A_{j, s}=a_{j, 1} \ldots a_{j, s-1} a_{j, s+1}^{-1} \ldots a_{j, 2 g}^{-1}$.

\section{Apresentação 2:}

Geradores: $\left\{A_{i, r} ; 1 \leq i \leq n-1,1 \leq r \leq 2 g\right\} \cup\left\{T_{j, k} ; 1 \leq j<k \leq n-1\right\}$.

Relações: as mesmas da apresentação 1, onde:

$$
\begin{aligned}
& a_{i, k}=\left(A_{i, 1} A_{i, 2}^{-1} \ldots A_{i, k-2} A_{i, k-1}^{-1}\right)\left(A_{i, k+1} A_{i, k+2}^{-1} \ldots A_{i, 2 g-1}^{-1} A_{i, 2 g}\right) \quad \text { se k for ímpar, } \\
& a_{i, k}^{-1}=\left(A_{i, 1} A_{i, 2}^{-1} \ldots A_{i, k-2}^{-1} A_{i, k-1}\right)\left(A_{i, k+1}^{-1} A_{i, k+2} \ldots A_{i, 2 g-1}^{-1} A_{i, 2 g}\right) \quad \text { se k for par. }
\end{aligned}
$$


Agora, vamos tomar a sequência exata (2):

$$
1 \longrightarrow \pi_{1}\left(M \backslash \mathcal{P}^{\prime}, P_{1}\right) \stackrel{u}{\longrightarrow} P B_{n}(M, \mathcal{P}) \stackrel{\nu}{\longrightarrow} P B_{n-1}\left(M, \mathcal{P}^{\prime}\right) \longrightarrow 1
$$

onde $u(\gamma)=\left(\gamma, e P_{2}, \ldots, e P_{n}\right)$, para cada $\gamma \in \pi_{1}\left(M \backslash \mathcal{P}^{\prime}, P_{1}\right)$ e $\nu(\Gamma)=\left(\gamma_{2}, \ldots, \gamma_{n}\right)$, para cada $\Gamma \in P B_{n}(M, \mathcal{P})$.

Para aplicar o Teorema 5.3.1, temos que conhecer as apresentações dos grupos à esquerda e à direita de $P B_{n}(M, \mathcal{P})$ na sequência exata (2). Para o grupo à esquerda, fazemos a seguinte afirmação:

Afirmação 1: O grupo fundamental $\pi_{1}\left(M \backslash \mathcal{P}^{\prime}, P_{1}\right)$ é livre e admite a seguinte apresentação: $<\left\{s_{1,1}, \ldots, s_{1,2 g}, t_{1,2}, \ldots, t_{1, n-1}\right\} ;->$. De fato, vamos considerar os abertos conexos por caminhos $U, V$ e $U \cap V$ representados na figura abaixo:

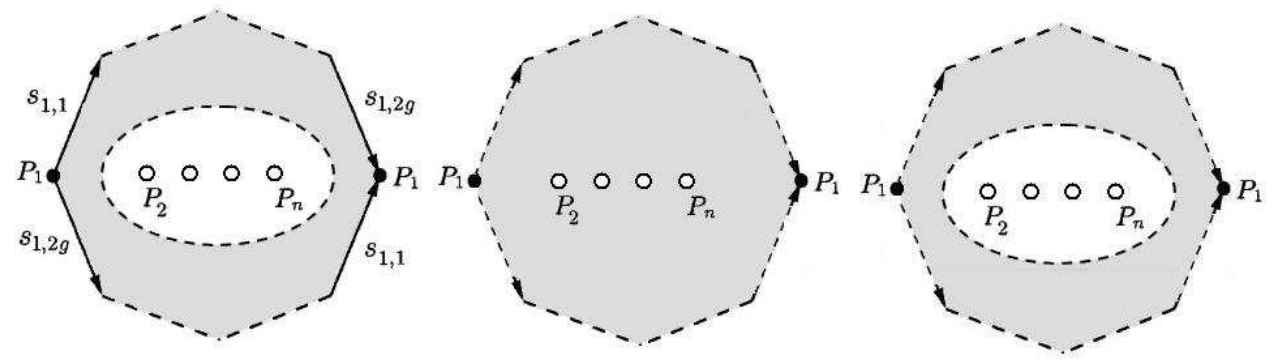

Figura 5.25: Os abertos $U, V$ e $U \cap V$.

Agora, aplicando o Teorema 3.1 .8 (versão 3 do Teorema de Seifert-Van Kampen) juntamente com o Teorema 3.1.5, temos:

$$
\pi_{1}\left(M \backslash \mathcal{P}^{\prime}, P_{1}\right) \simeq \frac{\pi_{1}\left(U, P_{1}\right) * \pi_{1}\left(V, P_{1}\right)}{N}
$$

O aberto $U$ possui o mesmo tipo de homotopia da soma conexa de $g$ toros menos um ponto. Logo, possui o mesmo tipo de homotopia da rosácea de $2 g$-pétalas. Portanto:

$$
\pi_{1}\left(U, P_{1}\right)=<\left\{s_{1,1}, \ldots, s_{1,2 g}\right\} ;->
$$

E também, o aberto $V$ possui o mesmo tipo de homotopia da rosácea de $(n-1)$-pétalas. Portanto:

$$
\pi_{1}\left(V, P_{1}\right)=<\left\{t_{1,2}, \ldots, t_{1, n}\right\} ;->
$$

Agora, vamos determinar $N$. Seja $[\gamma]$ um gerador de $\pi_{1}\left(U \cap V, P_{1}\right)$. Logo, $\left(i_{1}\right)_{*}[\gamma]=$ 
$\left[s_{1,1} \ldots s_{1,2 g} s_{1,1}^{-1} \ldots s_{1,2 g}^{-1}\right]$ e $\left(i_{2}\right)_{*}[\gamma]=\left[t_{1, n}\right]$. Mas pelo $P_{1}$-polígono abaixo temos:

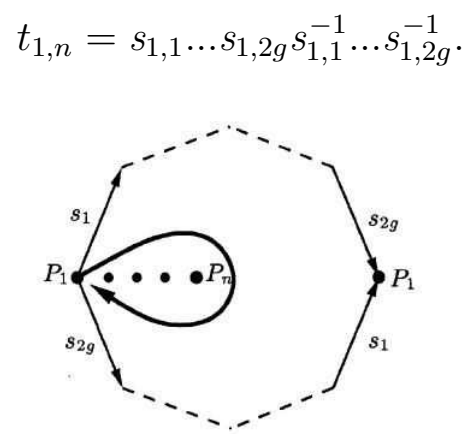

Figura 5.26: A corda $t_{1, n}$.

Logo, $\left(\left(i_{1}\right)_{*}[\gamma]\right) *\left(\left(i_{2}\right)_{*}[\gamma]\right)^{-1}=1$, ou seja, $N=\left[\left(\left(i_{1}\right)_{*}[\gamma]\right) *\left(\left(i_{2}\right)_{*}[\gamma]\right)^{-1}\right]=1$. Desde que $U \cap V$ tem o mesmo tipo de homotopia de $S^{1}$ e $\pi_{1}\left(S^{1}\right)$ é cíclico, é suficiente fazer o cálculo acima apenas para o gerador.

Assim, $\pi_{1}\left(M \backslash \mathcal{P}^{\prime}, P_{1}\right)=<\left\{s_{1,1}, \ldots, s_{1,2 g}, t_{1,2}, \ldots, t_{1, n}\right\} ;->$. Mas observemos que $t_{1, n}$ é escrito como combinação dos elementos do conjunto $\left\{s_{1,1}, \ldots, s_{1,2 g}\right\}$ e assim podemos remover $t_{1, n}$ dos geradores de $\pi_{1}\left(M \backslash \mathcal{P}^{\prime}, P_{1}\right)$. Portanto,

$$
\pi_{1}\left(M \backslash \mathcal{P}^{\prime}, P_{1}\right)=<\left\{s_{1,1}, \ldots, s_{1,2 g}, t_{1,2}, \ldots, t_{1, n-1}\right\} ;->
$$

e desta forma a afirmação fica provada.

Será bom para os nossos propósitos incluir $t_{1, n}$ entre os geradores. Então, vamos incluir uma única relação, a saber, a relação que extraímos do $P_{1}$-polígono anterior. Desta forma, vamos obter a seguinte apresentação:

$$
\pi_{1}\left(M \backslash \mathcal{P}^{\prime}, P_{1}\right)=<\left\{s_{1,1}, \ldots, s_{1,2 g}, t_{1,2}, \ldots, t_{1, n}\right\} ; t_{1, n}=s_{1,1} \ldots s_{1,2 g} s_{1,1}^{-1} \ldots s_{1,2 g}^{-1}>
$$

Logo, temos uma apresentação para o grupo da esquerda da sequência exata (2).

Para o grupo $P B_{n-1}(M)$ temos duas apresentações garantidas pela hipótese de indução. Agora, utilizaremos a apresentação 2. Desta forma, já podemos aplicar o Teorema 5.3.1 para conseguirmos uma apresentação para $P B_{n}(M, \mathcal{P})$.

Vamos observar que $\nu\left(a_{i, r}\right)=a_{i-1, r}$, para $i=2, \ldots, n$, então desde que $\nu$ é homomorfismo temos $\nu\left(A_{i, r}\right)=A_{i-1, r}$, para $i=2, \ldots, n$. Também, $\nu\left(T_{i, j}\right)=T_{i-1, j-1}$, para $2 \leq i \leq j \leq n$. Desta forma, conhecemos as pré-imagens por $\nu$ dos geradores de $P B_{n-1}\left(M, \mathcal{P}^{\prime}\right)$. Vamos observar também que $u\left(s_{1, r}\right)=a_{1, r}$ e $u\left(t_{1, j}\right)=T_{1, j}$ para todos os $r$ e $j$ possíveis. Então, obtemos imediatamente o seguinte conjunto de geradores para 
$P B_{n}(M, \mathcal{P})$

$$
\left\{a_{1, r} ; 1 \leq r \leq 2 g\right\} \cup\left\{A_{i, r} ; 2 \leq i \leq n, 1 \leq r \leq 2 g\right\} \cup\left\{T_{j, k} ; 1 \leq j<k \leq n\right\} .
$$

Ainda podemos simplificar este conjunto de geradores, aplicando o Lema 5.4.1, obtemos:

$$
\left\{a_{i, r} ; 1 \leq i \leq n, 1 \leq r \leq 2 g\right\} \cup\left\{T_{j, k} ; 1 \leq j<k \leq n\right\}
$$

que é a imagem por $\varphi$ do conjunto de geradores de $\overline{P B_{n}(M)}$. Em particular, $\varphi$ é sobrejetor.

Agora, vamos provar que $\varphi$ é um isomorfismo através do seguinte procedimento: vamos considerar as relações em $P B_{n}(M)$ que extrairemos do Teorema 5.3.1, e provar que as mesmas relações, quando consideradas em $\overline{P B_{n}(M)}$ ainda continuam válidas. Primeiro, vamos denotar por $G_{A}$ o conjunto de geradores de $\pi_{1}\left(M \backslash \mathcal{P}^{\prime}, P_{1}\right)$, e por $G$ o conjunto de geradores de $\overline{P B_{n}(M)}$. Consideremos a única relação na apresentação de $\pi_{1}\left(M \backslash \mathcal{P}^{\prime}, P_{1}\right)$, a qual consideramos uma relação em $P B_{n}(M)$ via o homomorfismo $u$. Esta será única relação de Tipo 1 na apresentação de $P B_{n}(M)$. O procedimento começa mostrando que esta relação é válida quando considerada em $\overline{P B_{n}(M)}$, isto é, temos uma relação em $\overline{P B_{n}(M)}$ a qual é aplicada por $\varphi$ na única relação na apresentação de $\pi_{1}\left(M \backslash \mathcal{P}^{\prime}, P_{1}\right)$.

Depois, para cada relator $r$ de $P B_{n-1}(M)$, vamos considerar a pré-imagem "canônica" por $\nu$ de $r$, denotada por $\tilde{r}$, no sentido do Teorema 5.3.1. Desde que $P B_{n}(M)$ e $\overline{P B_{n}(M)}$ possuem "os mesmos" geradores via o homomorfismo $\varphi$, podemos considerar também $\tilde{r}$ como uma palavra em $G$. Agora, encontramos uma palavra $U$ em $G$ tal que a igualdade $\tilde{r}=U$ é válida em $\overline{P B_{n}(M)}$, e tal que $\varphi(U)$ é uma palavra em $G_{A}$. Isto nos dará as relações de Tipo $2 \tilde{r}=\varphi(U)$ na apresentação de $P B_{n}(M)$.

Finalmente, para cada $x \in G_{A}$ e cada gerador $y$ de $P B_{n-1}(M)$, encontramos uma palavra $V$ em $G$ tal que a igualdade $\tilde{y} x \tilde{y}^{-1}=V$ é válida em $\overline{P B_{n}(M)}$, onde $\tilde{y}$ é a préimagem canônica por $\nu$ de $y$, e tal que $\varphi(V)$ é uma palavra em $G_{A}$. Isto nos dará as relações de Tipo 3 na apresentação de $P B_{n}(M)$.

Deste modo, teremos encontrado todas as relações de Tipo 1,2 e 3 do Teorema 5.3.1 e, portanto, uma apresentação de $P B_{n}(M)$ e, ao mesmo tempo,teremos mostrado que $\varphi$ é injetor e, consequentemente, $\varphi$ é um isomorfismo.

Vamos começar com o procedimento:

- Seja a única relação na apresentação de $\pi_{1}\left(M \backslash \mathcal{P}^{\prime}, P_{1}\right)$ :

$$
s_{1,1} \ldots s_{1,2 g} s_{1,1}^{-1} \ldots s_{1,2 g}^{-1}=t_{1, n} .
$$


Aplicando o homomorfismo $u$, temos:

$$
a_{1,1} \ldots a_{1,2 g} a_{1,1}^{-1} \ldots a_{1,2 g}^{-1}=T_{1, n}
$$

que corresponde à relação $(P R 8)$ de $\overline{P B_{n}(M)}$, para $j=1$. Então a relação é válida neste grupo. Esta é a única relação de Tipo 1.

- As relações de Tipo 2 são fáceis de encontrar:

Vamos começar com o relator de $(P R 1)$ em $P B_{n-1}(M)$ :

$$
a_{n-1,1}^{-1} a_{n-1,2}^{-1} \ldots a_{n-1,2 g}^{-1} a_{n-1,1} a_{n-1,2} \ldots a_{n-1,2 g}\left(\prod_{i=1}^{n-2} T_{i, n-2}^{-1} T_{i, n-1}\right)^{-1}
$$

que é igual a:

$$
\nu\left(a_{n, 1}^{-1}\right) \nu\left(a_{n, 2}^{-1}\right) \ldots \nu\left(a_{n, 2 g}^{-1}\right) \nu\left(a_{n, 1}\right) \nu\left(a_{n, 2}\right) \ldots \nu\left(a_{n, 2 g}\right) \nu\left(\left(\prod_{i=1}^{n-2} T_{i+1, n-1}^{-1} T_{i+1, n}\right)^{-1}\right)
$$

Assim, pela pré-imagem de $\nu$, obtemos $\tilde{r} \in P B_{n}(M)$ :

$$
\tilde{r}=a_{n, 1}^{-1} a_{n, 2}^{-1} \ldots a_{n, 2 g}^{-1} a_{n, 1} a_{n, 2} \ldots a_{n, 2 g}\left(\prod_{i=1}^{n-2} T_{i+1, n-1}^{-1} T_{i+1, n}\right)^{-1}
$$

Para nossa conveniência vamos fazer uma pequena mudança de índices no produtório em $\tilde{r}$ :

$$
\tilde{r}=a_{n, 1}^{-1} a_{n, 2}^{-1} \ldots a_{n, 2 g}^{-1} a_{n, 1} a_{n, 2} \ldots a_{n, 2 g}\left(\prod_{i=2}^{n-1} T_{i, n-1}^{-1} T_{i, n}\right)^{-1} .
$$

Por outro lado, pela relação $(P R 1)$ de $\overline{P B_{n}(M)}$, temos:

$$
a_{n, 1}^{-1} a_{n, 2}^{-1} \ldots a_{n, 2 g}^{-1} a_{n, 1} a_{n, 2} \ldots a_{n, 2 g}=\prod_{i=1}^{n-1} T_{i, n-1}^{-1} T_{i, n}
$$

que pode ser visto como

$$
a_{n, 1}^{-1} a_{n, 2}^{-1} \ldots a_{n, 2 g}^{-1} a_{n, 1} a_{n, 2} \ldots a_{n, 2 g}\left(\prod_{i=2}^{n-1} T_{i, n-1}^{-1} T_{i, n}\right)^{-1}=T_{1, n-1}^{-1} T_{1, n}
$$


Neste caso, temos $U=T_{1, n-1}^{-1} T_{1, n}$.

Notemos que $\tilde{r}$ pode ser visto como uma palavra em $G$ já que $P B_{n}(M)$ e $\overline{P B_{n}(M)}$ possuem os "mesmos" geradores e $\tilde{r}$ aparece na relação $(P R 1)$ de $P B_{n}(M)$. Logo, $\tilde{r}=U$, ou melhor, $\tilde{r}=\varphi(U)$. Mas $\varphi(U)=\varphi\left(T_{1, n-1}^{-1} T_{1, n}\right)=T_{1, n-1}^{-1} T_{1, n}=u\left(t_{1, n-1}^{-1} t_{1, n}\right)$, ou seja, uma palavra em $G_{A}$. Portanto,

$$
a_{n, 1}^{-1} a_{n, 2}^{-1} \ldots a_{n, 2 g}^{-1} a_{n, 1} a_{n, 2} \ldots a_{n, 2 g}\left(\prod_{i=2}^{n-1} T_{i, n-1}^{-1} T_{i, n}\right)^{-1}=T_{1, n-1}^{-1} T_{1, n}
$$

é uma das relações de Tipo 2 que estamos procurando. Mais ainda, esta relação é válida em $\overline{P B_{n}(M)}$ pois $\tilde{r}=\varphi(U)$ implica na relação $(P R 1)$ da apresentação de $\overline{P B_{n}(M)}$.

Agora, seja o relator de $(P R 2)$ de $P B_{n-1}(M)$ :

$$
a_{i, r} A_{j, s} a_{i, r}^{-1} A_{j, s}^{-1}
$$

Para nossa conveniência, vamos fazer a mudança de índices:

$$
a_{i-1, r} A_{j-1, s} a_{i-1, r}^{-1} A_{j-1, s}^{-1} \quad 0 \leq i-1 \leq j-1 \leq n-2,1 \leq r, s \leq 2 g, r \neq s .
$$

que é igual a:

$$
\nu\left(a_{i, r}\right) \nu\left(A_{j, s}\right) \nu\left(a_{i, r}^{-1}\right) \nu\left(A_{j, s}^{-1}\right), \quad i \geq 2 .
$$

Assim, pela pré-imagem de $\nu$, temos $\tilde{r} \in P B_{n}(M)$ :

$$
\tilde{r}=a_{i, r} A_{j, s} a_{i, r}^{-1} A_{j, s}^{-1}, \quad i \geq 2
$$

Por outro lado, pela relação $(P R 2)$ de $\overline{P B_{n}(M)}$, temos:

$$
a_{i, r} A_{j, s} a_{i, r}^{-1} A_{j, s}^{-1}=1, \quad i \geq 2 .
$$

Neste caso, temos $U=1$.

Notemos que $\tilde{r}$ pode ser visto como uma palavra em $G$ já que $P B_{n}(M)$ e $\overline{P B_{n}(M)}$ "possuem" os mesmos geradores e $\tilde{r}$ aparece na relação $(P R 2)$ de $P B_{n}(M)$. Logo, $\tilde{r}=U$, ou seja, $\tilde{r}=\varphi(U)$. Mas $\varphi(U)=\varphi(1)=1$, ou seja, uma palavra em $G_{A}$. 
Portanto,

$$
a_{i, r} A_{j, s} a_{i, r}^{-1} A_{j, s}^{-1}=1, \quad i \geq 2
$$

é uma das relações de Tipo 2 que estamos procurando. Mais ainda, esta relação é válida em $\overline{P B_{n}(M)}$ pois $\tilde{r}=\varphi(U)$ implica na relação $(P R 2)$ da apresentação de $\overline{P B_{n}(M)}$.

O raciocínio acima é usado para calcular ainda as relações $(P R 3),(P R 4)$ e $(P R 5)$ quando $i \geq 2$, (PR6) quando $i, j \geq 2$ e (PR7) quando $j \geq 2$ com $U$ sendo a palavra trivial para todas estas relações.

Agora, para finalizar consideremos o relator de $P B_{n-1}(M)$ :

$$
T_{j, n-1}\left(a_{j, 1} \ldots a_{j, 2 g} a_{j, 1}^{-1} \ldots a_{j, 2 g}^{-1}\right)^{-1}\left(\prod_{i=1}^{j-1} a_{i, 2 g}^{-1} \ldots a_{i, 1}^{-1} T_{i, j-1} T_{i, j}^{-1} a_{i, 1} \ldots a_{i, 2 g}\right)^{-1} .
$$

Fazendo uma mudança de índice em $j$ no relator acima obtemos:

$$
\begin{aligned}
& T_{j-1, n-1}\left(a_{j-1,1} \ldots a_{j-1,2 g} a_{j-1,1}^{-1} \ldots a_{j-1,2 g}^{-1}\right)^{-1}\left(\prod_{i=1}^{j-2} a_{i, 2 g}^{-1} \ldots a_{i, 1}^{-1} T_{i, j-2}\right. \\
& \left.T_{i, j-1}^{-1} a_{i, 1} \ldots a_{i, 2 g}\right)^{-1} .
\end{aligned}
$$

Agora mudando o índice $i$, obtemos:

$$
\begin{aligned}
& T_{j-1, n-1}\left(a_{j-1,1} \ldots a_{j-1,2 g} a_{j-1,1}^{-1} \ldots a_{j-1,2 g}^{-1}\right)^{-1}\left(\prod_{i=2}^{j-1} a_{i-1,2 g}^{-1} \ldots a_{i-1,1}^{-1} T_{i-1, j-2}\right. \\
& \left.T_{i-1, j-1}^{-1} a_{i-1,1} \ldots a_{i-1,2 g}\right)^{-1} .
\end{aligned}
$$

que é igual a:

$$
\begin{aligned}
& \nu\left(T_{j, n}\right) \nu\left(\left(a_{j-1,1} \ldots a_{j-1,2 g} a_{j-1,1}^{-1} \ldots a_{j-1,2 g}^{-1}\right)^{-1}\right) \nu\left(\left(\prod_{i=2}^{j} a_{i-1,2 g}^{-1} \ldots a_{i-1,1}^{-1} T_{i-1, j-2}\right.\right. \\
& \left.\left.T_{i-1, j-1}^{-1} a_{i-1,1} \ldots a_{i-1,2 g}\right)^{-1}\right), \quad j \geq 2 .
\end{aligned}
$$

Assim, pela pré-imagem de $\nu$, temos $\tilde{r} \in P B_{n}(M)$ :

$$
\tilde{r}=T_{j, n}\left(a_{j, 1} \ldots a_{j, 2 g} a_{j, 1}^{-1} \ldots a_{j, 2 g}^{-1}\right)^{-1}\left(\prod_{i=2}^{j} a_{i, 2 g}^{-1} \ldots a_{i, 1}^{-1} T_{i, j-1} T_{i, j}^{-1} a_{i, 1} \ldots a_{i, 2 g}\right)^{-1} .
$$


Por outro lado, pela relação $(P R 8)$ de $\overline{P B_{n}(M)}$, temos:

$$
\begin{aligned}
& T_{j, n}\left(a_{j, 1} \ldots a_{j, 2 g} a_{j, 1}^{-1} \ldots a_{j, 2 g}^{-1}\right)^{-1}\left(\prod_{i=2}^{j-1} a_{i, 2 g}^{-1} \ldots a_{i, 1}^{-1} T_{i, j-1} T_{i, j}^{-1} a_{i, 1} \ldots a_{i, 2 g}\right)^{-1}= \\
& a_{1,2 g}^{-1} \ldots a_{1,1}^{-1} T_{1, j-1} T_{1, j}^{-1} a_{1,1} \ldots a_{1,2 g} .
\end{aligned}
$$

Neste caso, temos $U=a_{1,2 g}^{-1} \ldots a_{1,1}^{-1} T_{1, j-1} T_{1, j}^{-1} a_{1,1} \ldots a_{1,2 g}$. Novamente, $\tilde{r}$ pode ser visto como uma palavra em $G$. Logo, $\tilde{r}=\varphi(U), \operatorname{com} \varphi(U)=a_{1,2 g}^{-1} \ldots a_{1,1}^{-1} T_{1, j-1} T_{1, j}^{-1} a_{1,1} \ldots a_{1,2 g}$ uma palavra em $G_{A}$. Portanto,

$$
\begin{aligned}
& T_{j, n}\left(a_{j, 1} \ldots a_{j, 2 g} a_{j, 1}^{-1} \ldots a_{j, 2 g}^{-1}\right)^{-1}\left(\prod_{i=2}^{j-1} a_{i, 2 g}^{-1} \ldots a_{i, 1}^{-1} T_{i, j-1} T_{i, j}^{-1} a_{i, 1} \ldots a_{i, 2 g}\right)^{-1}= \\
& a_{1,2 g}^{-1} \ldots a_{1,1}^{-1} T_{1, j-1} T_{1, j}^{-1} a_{1,1} \ldots a_{1,2 g} .
\end{aligned}
$$

é a relação de Tipo 2 que queríamos. E ainda é válida em $\overline{P B_{n}(M)}$ pelo mesmo argumento das relações anteriores.

- Finalmente, vamos encontrar as relações de Tipo 3:

Para encontrar tais relações, vamos considerar a apresentação 2 de $P B_{n-1}(M)$. Para cada gerador $x$ de $G_{A}$ e para cada gerador $y$ de $P B_{n-1}(M)$, lembrando que $\tilde{y}$ é a pré-imagem de $y$ por $\nu$, vamos ter as seguintes possibilidades para $\tilde{y} x \tilde{y}^{-1}$ :

$$
A_{i, r} a_{1, r} A_{i, r}^{-1}, A_{i, r} T_{1, j} A_{i, r}^{-1}, T_{j, k} a_{1, r} T_{j, k}^{-1} \text { e } T_{j, k} T_{1, j} T_{j, k}^{-1}
$$

onde $x$ neste produto é tal que $x=u(x)$.

Para $i=1$, temos pela relação $(P R 2)$ de $P B_{n-1}(M)$ que $A_{j, s} a_{1, r} A_{j, s}^{-1}=a_{1, r}$, com $r \neq s$. Temos também que $a_{1, r}$ é uma palavra em $G$ e $\varphi(V)=a_{1, r}$ é uma palavra em $G_{A}$. Assim, $V=a_{1, r}$. Mais ainda, $A_{j, s} a_{1, r} A_{j, s}^{-1}=a_{1, r}$ é válida em $\overline{P B_{n}(M)}$. Portanto, esta é a primeira relação de Tipo 3 que procurávamos.

Agora, seja a relação $(P R 3)$ de $P B_{n-1}(M) \operatorname{com} i=1$ :

$$
\begin{aligned}
T_{1, j} T_{1, j-1}^{-1} & =\left(a_{1,1} \ldots a_{1, r}\right) A_{j, r}\left(a_{1, r}^{-1} \ldots a_{1,1}^{-1}\right) A_{j, r}^{-1} \\
& =\left(a_{1,1} \ldots a_{1, r}\right) A_{j, r} a_{1, r}^{-1}\left(a_{1, r-1}^{-1} \ldots a_{1,1}^{-1}\right) A_{j, r}^{-1} .
\end{aligned}
$$

Logo:

$$
\left(a_{1, r}^{-1} \ldots a_{1,1}^{-1}\right) T_{1, j} T_{1, j-1}^{-1}=A_{j, r} a_{1, r}^{-1}\left(a_{1, r-1}^{-1} \ldots a_{1,1}^{-1}\right) A_{j, r}^{-1}
$$


Usando ( $P R 2)$ na equação acima, temos:

$$
A_{j, r} a_{1, r}^{-1} A_{j, r}^{-1}\left(a_{1, r-1}^{-1} \ldots a_{1,1}^{-1}\right)=\left(a_{1, r}^{-1} \ldots a_{1,1}^{-1}\right) T_{1, j} T_{1, j-1}^{-1} .
$$

Desta forma, chegamos a seguinte equação:

$$
A_{j, r} a_{1, r} A_{j, r}^{-1}=\left(a_{1, r-1}^{-1} \ldots a_{1,1}^{-1}\right) T_{1, j-1} T_{1, j}^{-1}\left(a_{1,1} \ldots a_{1, r}\right) .
$$

Vamos observar que o segundo membro dessa equação é uma palavra em $G$ e, aplicando o homomorfismo $\varphi$ nessa palavra obtemos ela mesma, que é uma palavra em $G_{A}$. Portanto, $\left(a_{1, r-1}^{-1} \ldots a_{1,1}^{-1}\right) T_{1, j} T_{1, j-1}^{-1}\left(a_{1,1} \ldots a_{1, r}\right)$ é a palavra $V$ que procuramos e, portanto, $A_{j, r} a_{1, r} A_{j, r}^{-1}=\left(a_{1, r-1}^{-1} \ldots a_{1,1}^{-1}\right) T_{1, j} T_{1, j-1}^{-1}\left(a_{1,1} \ldots a_{1, r}\right)$ é uma equação de Tipo 3 que é válida em $\overline{P B_{n}(M)}$ desde que a relação encontrada é uma particularidade da relação $(P R 3)$ de $\overline{P B_{n}(M)}$.

De modo totalmente análogo, as relações de Tipo 3 da forma $T_{k, l} T_{1, j} T_{k, l}^{-1}=V$, com $V$ sendo uma palavra em $G_{A}$ seguem das relações $(P R 4)$ e $(P R 5)$ de $P B_{n-1}(M)$ enquanto as relações da forma $T_{k, l} a_{1, r} T_{k, l}^{-1}=V$ seguem de (PR6) quando $i=1$. Também, se $j>k$ obtemos de $(P R 6)$ as relações de Tipo 3 da forma $A_{j, r} T_{1, k} A_{j, r}^{-1}=$ $V$ : de fato, notemos que $T_{1, k}$ é uma palavra em $G_{A}$. Tomemos $V=T_{1, k}$. Logo, $V=T_{1, k}=A_{j, r} A_{j, r}^{-1} T_{1, k}$. E desde que $j>k$, por $(P R 6), A_{j, r}^{-1}$ comuta com $V$. Assim, temos:

$$
A_{j, r} T_{1, k} A_{j, r}^{-1}=V
$$

que é ainda válida em $\overline{P B_{n}(M)}$.

Agora, as relações que faltam são aquelas da forma $A_{j, r} T_{1, k} A_{j, r}^{-1}=V$ quando $1<$ $j \leq k$, as quais são deduzidas como segue: por $(P R 7)$, sabemos que $a_{j, s}$ comuta com o elemento $a_{1,2 g}^{-1} \ldots a_{1,1}^{-1} T_{1, k} a_{1,2 g} \ldots a_{1,1}, s=1, \ldots, 2 g$. Logo, pela definição, temos que $A_{j, s}$ comuta com o mesmo elemento. Assim:

$$
\begin{aligned}
& \left(a_{1,2 g}^{-1} \ldots a_{1,1}^{-1} T_{1, k} a_{1,2 g} \ldots a_{1,1}\right)=A_{j, r}\left(a_{1,2 g}^{-1} \ldots a_{1,1}^{-1} T_{1, k} a_{1,2 g} \ldots a_{1,1}\right) A_{j, r}^{-1} \\
& =\left(A_{j, r} a_{1,2 g}^{-1} A_{j, r}^{-1}\right) \ldots\left(A_{j, r} a_{1,1}^{-1} A_{j, r}^{-1}\right)\left(A_{j, r} T_{1, k} A_{j, r}^{-1}\right)\left(A_{j, r} a_{1,2 g} A_{j, r}^{-1}\right) \ldots\left(A_{j, r} a_{1,1} A_{j, r}^{-1}\right) .
\end{aligned}
$$

Mas usando (PR2) e (PR3) sabemos como escrever todos os termos no produto acima (com excessão do termo do meio) como palavras em $G_{A}$. Assim, isolando o termo do meio num membro e definindo $V$ como os outros termos que estão no 
outro membro da equação teremos a equação de Tipo 3 que procurávamos.

E assim, encontramos todas as relações de Tipos 1,2 e 3 de $P B_{n}(M)$. Como todas as relações são válidas em $\overline{P B_{n}(M)}$, provamos ainda que $\varphi$ é injetora. Desta forma, temos o seguinte resultado:

Teorema 5.4.2. Se $M$ for uma superfície fechada, orientável de gênero $g \geq 1$ então $\mathrm{PB}_{n}(M)$ admitirá as Apresentações 1 e 2 definidas anteriormente como apresentações.

Passo 3. Agora, nós queremos encontrar uma apresentação para $B_{n}(M)$, onde $M$ é uma superfície orientável de gênero $g \geq 1$. Vamos definir o grupo $\overline{B_{n}(M)}$ dado pela apresentação do Teorema 5.2.2.

Esta é a menor apresentação que encontramos. Mas, para mostrar sua validade, vamos precisar modificá-la um pouco, obtendo uma nova apresentação com mais geradores e mais relações, mas equivalente a primeira.

Primeiro, mudaremos um pouco nossa notação e chamamos de $a_{1, r}$ os geradores $a_{r}$, para $r=1, \ldots, 2 g$. Então devemos simplesmente adicionar na apresentação dada os geradores:

- $a_{i, r}$, para $i=2, \ldots, n$ e $r=1, \ldots, 2 g$,

- $T_{j, k}$, para $1 \leq j<k \leq n$.

e as relações:

(R8) $a_{j+1, r}=\sigma_{j}^{-1} a_{j, r} \sigma_{j}^{-1}$, para $1 \leq j \leq n-1 ; 1 \leq r \leq 2 g ; r$ ímpar.

(R9) $T_{j, k}=\sigma_{j} \sigma_{j+1} \ldots \sigma_{k-2} \sigma_{k-1}^{2} \sigma_{k-2} \ldots \sigma_{j}$, para $1 \leq j<k \leq n$.

Claramente, ambas apresentações definem o mesmo grupo, isto é, $\overline{B_{n}(M)}$. Agora, vamos definir da forma mais natural possível:

$$
\begin{aligned}
\psi & : \overline{B_{n}(M)} \rightarrow B_{n}(M) \\
& \sigma_{i} \longmapsto \sigma_{i}, 1 \leq i \leq n-1 \\
& a_{i, r} \longmapsto a_{i, r}, 1 \leq i \leq n, 1 \leq r \leq 2 g
\end{aligned}
$$

É fácil de visualizar que as relações $(R 7),(R 8)$ e $(R 9)$ são válidas em $B_{n}(M)$ : de fato, para $(R 7)$, temos: 


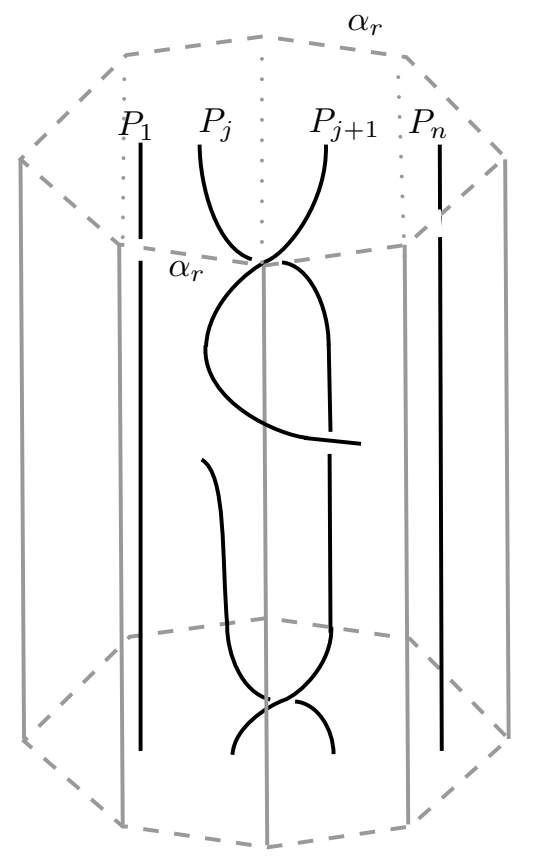

Portanto, a relação $(R 7)$ é válida em $B_{n}(M)$. Para $(R 8)$, o caso é totalmente análogo. A relação $(R 9)$ é claramente válida em $B_{n}(M)$ já que podemos facilmente representá-la como uma trança no disco:

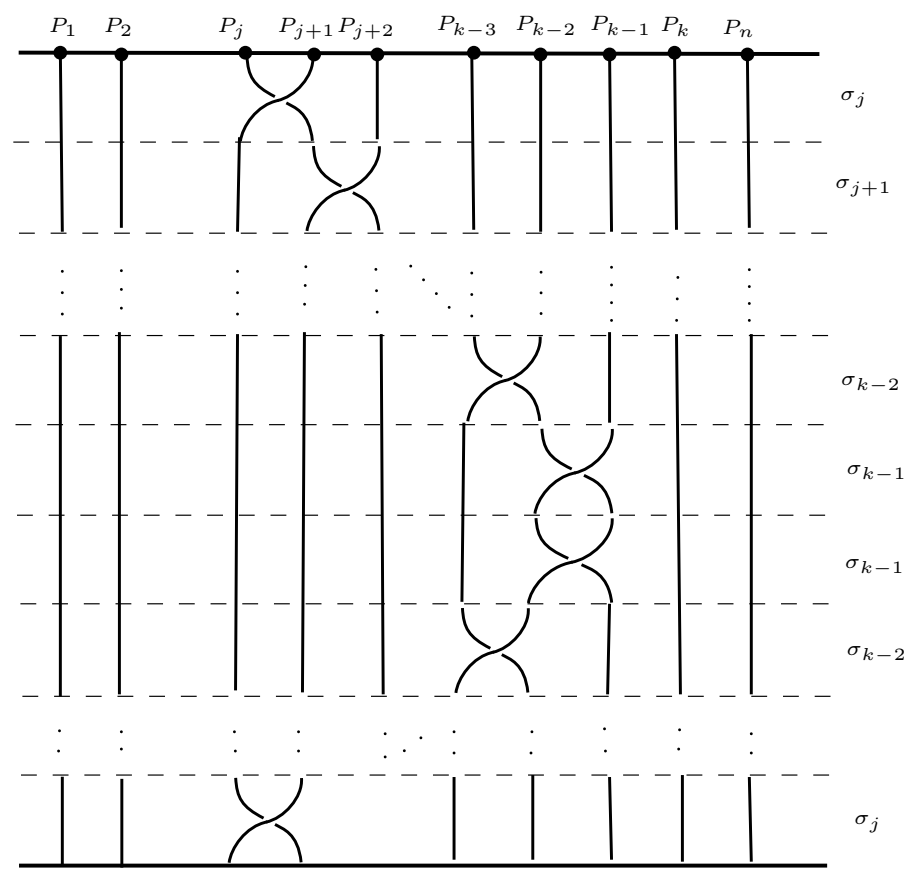

Também, mostramos que as relações $(R 1)$ - $(R 6)$ estão em $B_{n}(M)$ na primeira seção deste capítulo. Desta forma, temos que $\psi$ é um homomorfismo bem definido. 
Agora, consideremos a sequência exata (1):

$$
1 \longrightarrow P B_{n}(M) \stackrel{e}{\longrightarrow} B_{n}(M) \stackrel{f}{\longrightarrow} \Sigma_{n} \longrightarrow 1
$$

Pelo Teorema 5.4.2, temos duas apresentações para $P B_{n}(M)$ (e por ora, vamos considerar a apresentação 1) e para o grupo das permutações $\Sigma_{n}$ temos uma apresentação no artigo [H] (vide Proposição 8) que é dada abaixo:

\section{Apresentação de $\Sigma_{n}$ :}

Geradores: $\delta_{1}, \ldots, \delta_{n-1}$.

\section{Relações:}

$$
\begin{array}{lr}
-\delta_{i} \delta_{j}=\delta_{j} \delta_{i}, & |i-j| \geq 2, \\
-\delta_{i} \delta_{i+1} \delta_{i}=\delta_{i+1} \delta_{i} \delta_{i+1}, & 1 \leq i \leq n-2, \\
-\delta_{i}^{2}=1, & 1 \leq i \leq n-1,
\end{array}
$$

onde $\delta_{i}$ é a permutação $(i, i+1)$, para qualquer $i$.

Observemos que $\sigma_{i}$ é uma pré-imagem de $\delta_{i}$ por $f$. Logo, aplicando o Teorema 5.3.1, temos o seguinte conjunto de geradores para $B_{n}(M)$ :

$$
\left\{a_{i, r} ; 1 \leq i \leq n, 1 \leq r \leq 2 g\right\} \cup\left\{T_{j, k} ; 1 \leq j<k \leq n\right\} \cup\left\{\sigma_{i} ; i=1, \ldots, n-1\right\}
$$

Como $T_{j, k}$ pode ser escrito como combinação dos elementos de $\left\{\sigma_{i} ; i=1, \ldots, n-1\right\}$, podemos simplificar o conjunto de geradores de $B_{n}(M)$ :

$$
\left\{a_{i, r} ; 1 \leq i \leq n, 1 \leq r \leq 2 g\right\} \cup\left\{\sigma_{i} ; i=1, \ldots, n-1\right\}
$$

que é a imagem por $\psi$ dos geradores de $\overline{B_{n}(M)}$ e assim, $\psi$ é sobrejetor.

Analogamente ao que fizemos no passo 2, vamos mostrar que $\psi$ é um isomorfismo pelo procedimento a seguir:

Primeiro, vamos denotar por $G_{A}$ o conjunto de geradores de $P B_{n}(M)$, e por $G$ o conjunto de geradores de $\overline{B_{n}(M)}$. Para cada relação na apresentação de $P B_{n}(M)$, vamos considerá-las via e como relações em $B_{n}(M)$ e mostraremos que são válidas em $\overline{B_{n}(M)}$. 
Depois, para cada relator $r$ de $\Sigma_{n}$, vamos considerar sua pré-imagem canônica por $f$, denotada por $\tilde{r}$. Então, encontramos uma palavra $U$ em $G$ tal que $\tilde{r}=U$ é válido em $\overline{B_{n}(M)}$, e tal que $\psi(U)$ é uma palavra em $G_{A}$.

Finalmente, para cada $x \in G_{A}$ e cada gerador $\delta_{i}$ de $\Sigma_{n}$, encontramos uma palavra $V$ em $G$ tal que a igualdade $\sigma_{i} x \sigma_{i}^{-1}=V$ é válida em $\overline{B_{n}(M)}$ e tal que $\psi(V)$ é uma palavra em $G_{A}$.

Isto nos dá as relações de Tipo 1, 2 e 3 do Teorema 5.3.1 e, portanto, uma apresentação de $B_{n}(M)$, e, ao mesmo tempo isto mostra que $\psi$ é injetora, e, consequentemente, que $\psi$ é um isomorfismo.

- Vamos então verificar em $\overline{B_{n}(M)}$ as relações de Tipo 1. Partiremos de (R3) para chegar na relação $(P R 1)$ :

$$
a_{1,1} \ldots a_{1,2 g} a_{1,1}^{-1} \ldots a_{1,2 g}^{-1}=\sigma_{1} \ldots \sigma_{n-2} \sigma_{n-1}^{2} \sigma_{n-2} \ldots \sigma_{1}
$$

Substituindo cada $a_{i, r}, i=1, \ldots, n-1$ por $(R 7)$ quando $r$ for ímpar e por $(R 8)$ quando $r$ for par, temos:

$$
\begin{aligned}
\sigma_{1} \ldots \sigma_{n-2} \sigma_{n-1}^{2} \sigma_{n-2} \ldots \sigma_{1} & =a_{1,1} \ldots a_{1,2 g} a_{1,1}^{-1} \ldots a_{1,2 g}^{-1} \\
& =\sigma_{1}\left(a_{2,1} \ldots a_{2,2 g}\right) \sigma_{1}^{-2}\left(a_{2,1}^{-1} \ldots a_{2,2 g}^{-1}\right) \sigma_{1} \\
& =\sigma_{1} \ldots \sigma_{n-1}\left(a_{n, 1} \ldots a_{n, 2 g}\right) \sigma_{n-1}^{-1} \ldots \sigma_{1}^{-2} \ldots \sigma_{n-1}^{-1}\left(a_{n, 1}^{-1} \ldots a_{n, 2 g}^{-1}\right) \sigma_{n-1} \ldots \sigma_{1} .
\end{aligned}
$$

Por outro lado, por $(R 9)$, temos:

$$
\begin{aligned}
T_{i, n-1}^{-1} & =\left(\sigma_{i} \sigma_{i+1} \ldots \sigma_{n-3} \sigma_{n-2}^{2} \sigma_{n-3} \ldots \sigma_{i+1} \sigma_{i}\right)^{-1} \\
& =\sigma_{i}^{-1} \ldots \sigma_{n-3}^{-1} \sigma_{n-2}^{-2} \sigma_{n-3}^{-1} \ldots \sigma_{i}^{-1} \\
T_{i, n} & =\sigma_{i} \sigma_{i+1} \ldots \sigma_{n-2} \sigma_{n-1}^{2} \sigma_{n-2} \ldots \sigma_{i+1} \sigma_{i}
\end{aligned}
$$

Portanto,

$$
\begin{aligned}
T_{i, n-1}^{-1} T_{i, n} & =\left(\sigma_{i}^{-1} \ldots \sigma_{n-3}^{-1} \sigma_{n-2}^{-2} \sigma_{n-3}^{-1} \ldots \sigma_{i}^{-1}\right)\left(\sigma_{i} \sigma_{i+1} \ldots \sigma_{n-2} \sigma_{n-1}^{2} \sigma_{n-2} \ldots \sigma_{i+1} \sigma_{i}\right) \\
& =\sigma_{i}^{-1} \ldots \sigma_{n-3}^{-1} \sigma_{n-2}^{-1} \sigma_{n-1}^{2} \sigma_{n-2} \ldots \sigma_{i} \\
& =\sigma_{i}^{-1} \sigma_{i+1}^{-1} \ldots \sigma_{n-2}^{-1} \sigma_{n-1}^{2} \sigma_{n-2} \ldots \sigma_{i+1} \sigma_{i}
\end{aligned}
$$


E ainda, usando $(R 1)$ e $(R 2)$ :

$$
\begin{aligned}
T_{i, n-1}^{-1} T_{i, n} & =\sigma_{i}^{-1} \sigma_{i+1}^{-1} \ldots \sigma_{n-2}^{-1} \sigma_{n-1}^{2} \sigma_{n-2} \ldots \sigma_{i+1} \sigma_{i} \\
& =\sigma_{i}^{-1} \sigma_{i+1}^{-1} \ldots \sigma_{n-2}^{-1} \sigma_{n-1} \sigma_{n-1} \sigma_{n-2} \ldots \sigma_{i+1} \sigma_{i} \\
& =\sigma_{i}^{-1} \sigma_{i+1}^{-1} \ldots \sigma_{n-2}^{-1} \sigma_{n-1} \sigma_{n-2} \sigma_{n-1} \sigma_{n-2} \sigma_{n-1}^{-1} \ldots \sigma_{i+1} \sigma_{i} \\
& =\sigma_{i}^{-1} \sigma_{i+1}^{-1} \ldots \sigma_{n-2}^{-1} \sigma_{n-2} \sigma_{n-1} \sigma_{n-2} \sigma_{n-2} \sigma_{n-1}^{-1} \ldots \sigma_{i+1} \sigma_{i} \\
& =\sigma_{i}^{-1} \sigma_{i+1}^{-1} \ldots \sigma_{n-1} \sigma_{n-2}^{2} \sigma_{n-1}^{-1} \ldots \sigma_{i+1} \sigma_{i} \\
& =\sigma_{n-1} \sigma_{i}^{-1} \sigma_{i+1}^{-1} \ldots \sigma_{n-3}^{-1} \sigma_{n-2}^{2} \sigma_{n-1}^{-1} \ldots \sigma_{i} \\
& =\sigma_{n-1} \sigma_{i}^{-1} \sigma_{i+1}^{-1} \ldots \sigma_{n-3}^{-1} \sigma_{n-2} \sigma_{n-2} \ldots \sigma_{i+1} \sigma_{i} \sigma_{n-1}^{-1} \\
& =\sigma_{n-1} \sigma_{i}^{-1} \sigma_{i+1}^{-1} \ldots \sigma_{n-3}^{-1} \sigma_{n-2} \sigma_{n-2} \sigma_{n-3} \ldots \sigma_{i+1} \sigma_{i} \sigma_{n-1}^{-1} \\
& =\sigma_{n-1} \sigma_{i}^{-1} \sigma_{i+1}^{-1} \ldots \sigma_{n-3}^{-1} \sigma_{n-2} \sigma_{n-3} \sigma_{n-2} \sigma_{n-3} \sigma_{n-2}^{-1} \ldots \sigma_{i+1} \sigma_{i} \sigma_{n-1}^{-1} \\
& =\sigma_{n-1} \sigma_{i}^{-1} \sigma_{i+1}^{-1} \ldots \sigma_{n-3}^{-1} \sigma_{n-3} \sigma_{n-2} \sigma_{n-3} \sigma_{n-3} \sigma_{n-2}^{-1} \ldots \sigma_{i+1} \sigma_{i} \sigma_{n-1}^{-1} \\
& =\sigma_{n-1} \ldots \sigma_{i+1} \sigma_{i}^{2} \sigma_{i+1}^{-1} \ldots \sigma_{n-1}^{-1} .
\end{aligned}
$$

A partir da equação encontrada acima, obtemos:

$$
\begin{aligned}
\prod_{i=1}^{n-1} T_{i, n-1}^{-1} T_{i, n} & =\left(T_{1, n-1}^{-1} T_{1, n}\right)\left(T_{2, n-1}^{-1} T_{2, n}\right) \ldots\left(T_{n-2, n-1}^{-1} T_{n-2, n}\right)\left(T_{n-1, n-1}^{-1} T_{n-1, n}\right) \\
& =\sigma_{n-1} \ldots \sigma_{1}^{2} \ldots \sigma_{n-1} .
\end{aligned}
$$

E finalmente, substituindo este resultado na equação $(*)$, temos:

$$
a_{n, 1} \ldots a_{n, 2 g}\left(\prod_{i=1}^{n-1} T_{i, n-1}^{-1} T_{i, n}\right)^{-1} a_{n, 1}^{-1} \ldots a_{n, 2 g}^{-1}=1 .
$$

Um simples ajuste nos membros dessa última equação fornece a relação $(P R 1)$. Ou seja, de uma relação de $\overline{B_{n}(M)}$ retiramos a relação $(P R 1)$. Portanto, $(P R 1)$ é válida em $\overline{B_{n}(M)}$. Mais ainda, via homomorfismo $e,(P R 1)$ é uma relação do Tipo 1 em $B_{n}(M)$. Daqui para frente usaremos algumas relações de $\overline{B_{n}(M)}$ facilmente deduzidas de $(R 1)-(R 9)$. 
Para a primeira relação suponhamos $r$ ímpar. Assim, usando $(R 8)$, temos:

$$
\begin{aligned}
\left(\sigma_{i-1}^{-1} \ldots \sigma_{1}^{-1}\right) a_{1, r}\left(\sigma_{1}^{-1} \ldots \sigma_{i-1}^{-1}\right) & =\sigma_{i-1}^{-1} \ldots \sigma_{2}^{-1}\left(\sigma_{1}^{-1} a_{1, r} \sigma_{1}^{-1}\right) \sigma_{2}^{-1} \ldots \sigma_{i-1}^{-1} \\
& =\cdots \\
& =\sigma_{i-1}^{-1} a_{i-1, r} \sigma_{i-1}^{-1} \\
& =a_{i, r}
\end{aligned}
$$

Portanto,

$$
a_{i, r}=\left(\sigma_{i-1}^{-1} \ldots \sigma_{1}^{-1}\right) a_{1, r}\left(\sigma_{1}^{-1} \ldots \sigma_{i-1}^{-1}\right), \text { se } r \text { for ímpar. }
$$

De maneira totalmente análoga, usando $(R 7)$ obtemos:

$$
a_{i, r}=\left(\sigma_{i-1} \ldots \sigma_{1}\right) a_{1, r}\left(\sigma_{1} \ldots \sigma_{i-1}\right), \text { se } r \text { for par. }
$$

Outras duas relações são obtidas de $(R 7)$ e $(R 8)$. Suponhamos que $r$ seja ímpar. Logo,

$$
\begin{aligned}
& \left(\sigma_{j-1}^{-1} \ldots \sigma_{2}^{-1}\right) A_{2, s}\left(\sigma_{2}^{-1} \ldots \sigma_{j-1}^{-1}\right)=\left(\sigma_{j-1}^{-1} \ldots \sigma_{2}^{-1}\right)\left(a_{2,1} \ldots a_{2, s-1} a_{2, s+1}^{-1} \ldots a_{2,2 g}^{-1}\right)\left(\sigma_{2}^{-1} \ldots \sigma_{j-1}^{-1}\right) \\
& =\sigma_{j-1}^{-1} \ldots \sigma_{2}^{-1}\left(\sigma_{2} a_{3,1} \sigma_{2}\right)\left(\sigma_{2}^{-1} a_{3,2} \sigma_{2}^{-1}\right) \ldots\left(\sigma_{2}^{-1} a_{3,2 g} \sigma_{2}^{-1}\right)^{-1}\left(\sigma_{2}^{-1} \ldots \sigma_{j-1}^{-1}\right) \\
& =\left(\sigma_{j-1}^{-1} \ldots \sigma_{3}^{-1}\right)\left(a_{3,1} \ldots a_{3, s-1} a_{3, s+1}^{-1} \ldots a_{3,2 g}^{-1}\right)\left(\sigma_{3}^{-1} \ldots \sigma_{j-1}^{-1}\right) \\
& =\left(\sigma_{j-1}^{-1} \ldots \sigma_{3}^{-1}\right) A_{3, s}\left(\sigma_{3}^{-1} \ldots \sigma_{j-1}^{-1}\right) \\
& =\cdots \\
& =A_{j, s}
\end{aligned}
$$

E também:

$$
\begin{aligned}
& \left(\sigma_{j-1}^{-1} \ldots \sigma_{1}^{-1}\right) A_{1, s}\left(\sigma_{1}^{-1} \ldots \sigma_{j-1}^{-1}\right)=\left(\sigma_{j-1}^{-1} \ldots \sigma_{1}^{-1}\right)\left(a_{1,1} \ldots a_{1, s-1} a_{1, s+1}^{-1} \ldots a_{1,2 g}^{-1}\right)\left(\sigma_{1}^{-1} \ldots \sigma_{j-1}^{-1}\right) \\
& =\sigma_{j-1}^{-1} \ldots \sigma_{1}^{-1}\left(\sigma_{1} a_{2,1} \sigma_{1}\right)\left(\sigma_{1}^{-1} a_{2,2} \sigma_{1}^{-1}\right) \ldots\left(\sigma_{1}^{-1} a_{2,2 g} \sigma_{1}^{-1}\right)\left(\sigma_{1}^{-1} \ldots \sigma_{j-1}^{-1}\right) \\
& =\left(\sigma_{j-1}^{-1} \ldots \sigma_{2}^{-1}\right)\left(a_{2,1} \ldots a_{2, s-1} a_{2, s+1}^{-1} \ldots a_{2,2 g}^{-1}\right)\left(\sigma_{2}^{-1} \ldots \sigma_{j-1}^{-1}\right) \\
& =\left(\sigma_{j-1}^{-1} \ldots \sigma_{2}^{-1}\right) A_{2, s}\left(\sigma_{2}^{-1} \ldots \sigma_{j-1}^{-1}\right) \\
& =\cdots \\
& =A_{j, s}
\end{aligned}
$$


De forma totalmente análoga, provamos os casos acima quando $s$ for par.

Portanto,

$$
A_{j, s}=\left(\sigma_{j-1}^{-1} \ldots \sigma_{2}^{-1}\right) A_{2, s}\left(\sigma_{2}^{-1} \ldots \sigma_{j-1}^{-1}\right)=\left(\sigma_{j-1}^{-1} \ldots \sigma_{1}^{-1}\right) A_{1, s}\left(\sigma_{1}^{-1} \ldots \sigma_{j-1}^{-1}\right)
$$

Agora, das relações $(R 1)$ e $(R 2)$ (relações do grupo de tranças no disco) temos as seguintes relações (quando $i \leq j<k$ ):

$$
\begin{aligned}
\sigma_{j}\left(\sigma_{k} \sigma_{k-1} \ldots \sigma_{i}\right) & =\sigma_{k} \sigma_{j} \sigma_{k-1} \ldots \sigma_{j} \ldots \sigma_{i} \\
& =\sigma_{k} \sigma_{k-1} \sigma_{j} \ldots \sigma_{j} \ldots \sigma_{i} \\
& =\sigma_{k} \sigma_{k-1} \ldots \sigma_{j} \sigma_{j+1} \sigma_{j} \ldots \sigma_{i} \\
& =\sigma_{k} \sigma_{k-1} \ldots \sigma_{j+1} \sigma_{j} \sigma_{j+1} \ldots \sigma_{i} \\
& =\left(\sigma_{k} \sigma_{k-1} \ldots \sigma_{j+1} \sigma_{j} \ldots \sigma_{i}\right) \sigma_{j+1}
\end{aligned}
$$

Ou seja,

$$
\sigma_{j}\left(\sigma_{k} \sigma_{k-1} \ldots \sigma_{i}\right)=\left(\sigma_{k} \sigma_{k-1} \ldots \sigma_{i}\right) \sigma_{j+1}, \quad(i \leq j<k)
$$

E, pela relação (7) encontrada, obtemos a seguinte relação:

$$
\sigma_{j}\left(\sigma_{k}^{-1} \sigma_{k-1}^{-1} \ldots \sigma_{i}^{-1}\right)=\left(\sigma_{k}^{-1} \sigma_{k-1}^{-1} \ldots \sigma_{i}^{-1}\right) \sigma_{j+1}, \quad(i \leq j<k)
$$

Agora, de (7) e (8), temos a relação:

$$
\sigma_{i} \ldots \sigma_{k-1} \sigma_{k}^{2} \sigma_{k-1}^{-1} \ldots \sigma_{i}^{-1}=\sigma_{k}^{-1} \ldots \sigma_{i+1}^{-1} \sigma_{i}^{2} \sigma_{i+1} \ldots \sigma_{k}
$$

Agora, usando (6), (7), (8) e $(R 6)$, vemos que se $1 \leq k \leq j-2$ então:

$$
\begin{aligned}
\sigma_{k} A_{j, s} & =\sigma_{k}\left(\sigma_{j-1}^{-1} \ldots \sigma_{1}^{-1}\right) A_{1, s}\left(\sigma_{1}^{-1} \ldots \sigma_{j-1}^{-1}\right) \\
& =\left(\sigma_{j-1}^{-1} \ldots \sigma_{1}^{-1}\right) \sigma_{k+1} A_{1, s}\left(\sigma_{1}^{-1} \ldots \sigma_{j-1}^{-1}\right) \\
& =\left(\sigma_{j-1}^{-1} \ldots \sigma_{1}^{-1}\right) \sigma_{k+1}\left(a_{1,1} \ldots a_{1, r-1} a_{1, r+1}^{-1} \ldots a_{1,2 g}^{-1}\right)\left(\sigma_{1}^{-1} \ldots \sigma_{j-1}^{-1}\right) \\
& =\left(\sigma_{j-1}^{-1} \ldots \sigma_{1}^{-1}\right)\left(a_{1,1} \ldots a_{1, r-1} a_{1, r+1}^{-1} \ldots a_{1,2 g}^{-1}\right) \sigma_{k+1}\left(\sigma_{1}^{-1} \ldots \sigma_{j-1}^{-1}\right) \\
& =\left(\sigma_{j-1}^{-1} \ldots \sigma_{1}^{-1}\right) A_{1, s} \sigma_{k+1}\left(\sigma_{1}^{-1} \ldots \sigma_{j-1}^{-1}\right) \\
& =\left(\sigma_{j-1}^{-1} \ldots \sigma_{1}^{-1}\right) A_{1, s}\left(\sigma_{1}^{-1} \ldots \sigma_{j-1}^{-1}\right) \sigma_{k+1} \\
& =A_{j, s} \sigma_{k} .
\end{aligned}
$$


Ou seja,

$$
\sigma_{k} A_{j, s}=A_{j, s} \sigma_{k}
$$

Da mesma forma, usando $(6),(R 4)$ e $(R 6)$, temos para $r \neq s, 1<j$ :

$$
\begin{aligned}
a_{1, r} A_{j, s} & =a_{1, r}\left(\sigma_{j-1}^{-1} \ldots \sigma_{2}^{-1}\right) A_{2, s}\left(\sigma_{2}^{-1} \ldots \sigma_{j-1}^{-1}\right) \\
& =\left(\sigma_{j-1}^{-1} \ldots \sigma_{2}^{-1}\right) a_{1, r} A_{2, s}\left(\sigma_{2}^{-1} \ldots \sigma_{j-1}^{-1}\right) \\
& =\left(\sigma_{j-1}^{-1} \ldots \sigma_{2}^{-1}\right) a_{1, r}\left(a_{2,1} \ldots a_{2, r-1} a_{2, r+1}^{-1} \ldots a_{2,2 g}^{-1}\right)\left(\sigma_{2}^{-1} \ldots \sigma_{j-1}^{-1}\right) \\
& =\left(\sigma_{j-1}^{-1} \ldots \sigma_{2}^{-1}\right)\left(a_{2,1} \ldots a_{2, r-1} a_{2, r+1}^{-1} \ldots a_{2,2 g}^{-1}\right) a_{1, r}\left(\sigma_{2}^{-1} \ldots \sigma_{j-1}^{-1}\right) \\
& =\left(\sigma_{j-1}^{-1} \ldots \sigma_{2}^{-1}\right) A_{2, s}\left(\sigma_{2}^{-1} \ldots \sigma_{j-1}^{-1}\right) a_{1, r} \\
& =A_{j, s} a_{1, r} .
\end{aligned}
$$

E como por (4) e (5) $a_{i, r}$ é um produto de elementos que comuta com $A_{j, s}$, obtemos:

$$
a_{i, r} A_{j, s}=A_{j, s} a_{i, r}, i<j, r \neq s
$$

ou seja, $(P R 2)$ é válida em $\overline{B_{n}(M)}$. Mais ainda, $(P R 2)$ é uma relação de Tipo 1 via o homomorfismo e para $B_{n}(M)$. Agora, vamos verificar a relação $(P R 3)$. Faremos o caso em que $r$ é ímpar, sendo o outro caso totalmente análogo. Em cada uma das igualdades a seguir serão usadas as relações conhecidas de $\overline{B_{n}(M)}$ :

$$
\begin{aligned}
& T_{i, j} T_{i, j-1}^{-1} A_{j, r}\left(a_{i, 1} \ldots a_{i, r}\right) \\
& =\left(\sigma_{i} \ldots \sigma_{j-2} \sigma_{j-1}^{2} \sigma_{j-2}^{-1} \ldots \sigma_{i}^{-1}\right) A_{j, r}\left(a_{i, 1} \ldots a_{i, r}\right) \\
& =\left(\sigma_{i} \ldots \sigma_{j-2} \sigma_{j-1}^{2} \sigma_{j-2}^{-1} \ldots \sigma_{i}^{-1}\right) A_{j, r}\left(\sigma_{i-1}^{-1} \ldots \sigma_{1}^{-1}\right)\left(a_{1,1} \ldots a_{1, r}\right)\left(\sigma_{1}^{-1} \ldots \sigma_{i-1}^{-1}\right) \\
& =\left(\sigma_{i} \ldots \sigma_{j-2} \sigma_{j-1}^{2} \sigma_{j-2}^{-1} \ldots \sigma_{i}^{-1}\right)\left(\sigma_{i-1}^{-1} \ldots \sigma_{1}^{-1}\right) A_{j, r}\left(a_{1,1} \ldots a_{1, r}\right)\left(\sigma_{1}^{-1} \ldots \sigma_{i-1}^{-1}\right) \\
& =\left(\sigma_{i} \ldots \sigma_{j-2} \sigma_{j-1}^{2} \sigma_{j-2}^{-1} \ldots \sigma_{1}^{-1}\right) A_{j, r}\left(a_{1,1} \ldots a_{1, r}\right)\left(\sigma_{1}^{-1} \ldots \sigma_{i-1}^{-1}\right) \\
& =\left(\sigma_{i} \ldots \sigma_{j-2} \sigma_{j-1}^{2} \sigma_{j-2}^{-1} \ldots \sigma_{1}^{-1}\right)\left(\sigma_{j-1}^{-1} \ldots \sigma_{2}^{-1}\right) A_{2, r}\left(\sigma_{2}^{-1} \ldots \sigma_{j-1}^{-1}\right)\left(a_{1,1} \ldots a_{1, r}\right)\left(\sigma_{1}^{-1} \ldots \sigma_{i-1}^{-1}\right) \\
& =\left(\sigma_{i-1}^{-1} \ldots \sigma_{1}^{-1}\right)\left(\sigma_{1} \ldots \sigma_{i-1}\right)\left(\sigma_{i} \ldots \sigma_{j-2} \sigma_{j-1}^{2} \sigma_{j-2}^{-1} \ldots \sigma_{1}^{-1}\right)\left(\sigma_{j-1}^{-1} \ldots \sigma_{2}^{-1}\right) A_{2, r}\left(a_{1,1} \ldots a_{1, r}\right) \\
& \left(\sigma_{2}^{-1} \ldots \sigma_{j-1}^{-1}\right)\left(\sigma_{1}^{-1} \ldots \sigma_{i-1}^{-1}\right) \\
& =\left(\sigma_{i-1}^{-1} \ldots \sigma_{1}^{-1}\right)\left(\sigma_{j-1}^{-1} \ldots \sigma_{2}^{-1} \sigma_{1}^{2}\right) A_{2, r}\left(a_{1,1} \ldots a_{1, r}\right)\left(\sigma_{2}^{-1} \ldots \sigma_{j-1}^{-1}\right)\left(\sigma_{1}^{-1} \ldots \sigma_{i-1}^{-1}\right) \\
& =\left(\sigma_{i-1}^{-1} \ldots \sigma_{1}^{-1}\right)\left(\sigma_{j-1}^{-1} \ldots \sigma_{2}^{-1}\right)\left(a_{1,1} \ldots a_{1, r}\right) A_{2, r}\left(\sigma_{2}^{-1} \ldots \sigma_{j-1}^{-1}\right)\left(\sigma_{1}^{-1} \ldots \sigma_{i-1}^{-1}\right) \\
& =\left(\sigma_{i-1}^{-1} \ldots \sigma_{1}^{-1}\right)\left(a_{1,1} \ldots a_{1, r}\right)\left(\sigma_{j-1}^{-1} \ldots \sigma_{2}^{-1}\right) A_{2, r}\left(\sigma_{2}^{-1} \ldots \sigma_{j-1}^{-1}\right)\left(\sigma_{1}^{-1} \ldots \sigma_{i-1}^{-1}\right) \\
& =\left(\sigma_{i-1}^{-1} \ldots \sigma_{1}^{-1}\right)\left(a_{1,1} \ldots a_{1, r}\right) A_{j, r}\left(\sigma_{1}^{-1} \ldots \sigma_{i-1}^{-1}\right)
\end{aligned}
$$




$$
\begin{aligned}
& =\left(\sigma_{i-1}^{-1} \ldots \sigma_{1}^{-1}\right)\left(a_{1,1} \ldots a_{1, r}\right)\left(\sigma_{1}^{-1} \ldots \sigma_{i-1}^{-1}\right) A_{j, r} \\
& =\left(a_{i, 1} \ldots a_{i, r}\right) A_{j, r} .
\end{aligned}
$$

E portanto, temos (PR3). As relações $(P R 4)$ e $(P R 5)$ são tranças que não atravessam paredes, e assim são relações no grupo de tranças no disco, logo são consequências de $(R 1)$ e $(R 2)$. Agora, vamos mostrar que $(P R 6)$ é válido em $\overline{B_{n}(M)}$ : vamos fazer o caso em que $r$ é ímpar e $1 \leq j<k<i \leq n$ e os outros casos são totalmente análogos.

$$
\begin{aligned}
a_{i, r} T_{j, k} & =\left(\sigma_{i-1}^{-1} \ldots \sigma_{1}^{-1}\right) a_{1, r}\left(\sigma_{1}^{-1} \ldots \sigma_{i-1}^{-1}\right) T_{j, k} \\
& =\left(\sigma_{i-1}^{-1} \ldots \sigma_{1}^{-1}\right) a_{1, r}\left(\sigma_{1}^{-1} \ldots \sigma_{i-1}^{-1}\right)\left(\sigma_{j} \sigma_{j+1} \ldots \sigma_{k-2} \sigma_{k-1}^{2} \sigma_{k-2} \ldots \sigma_{j+1} \sigma_{j}\right) \\
& =\left(\sigma_{i-1}^{-1} \ldots \sigma_{1}^{-1}\right) a_{1, r}\left(\sigma_{j} \sigma_{j+1} \ldots \sigma_{k-2} \sigma_{k-1}^{2} \sigma_{k-2} \ldots \sigma_{j+1} \sigma_{j}\right)\left(\sigma_{1}^{-1} \ldots \sigma_{i-1}^{-1}\right) \\
& =\left(\sigma_{i-1}^{-1} \ldots \sigma_{1}^{-1}\right)\left(\sigma_{j} \sigma_{j+1} \ldots \sigma_{k-2} \sigma_{k-1}^{2} \sigma_{k-2} \ldots \sigma_{j+1} \sigma_{j}\right) a_{1, r}\left(\sigma_{1}^{-1} \ldots \sigma_{i-1}^{-1}\right) \\
& =\left(\sigma_{j} \sigma_{j+1} \ldots \sigma_{k-2} \sigma_{k-1}^{2} \sigma_{k-2} \ldots \sigma_{j+1} \sigma_{j}\right)\left(\sigma_{i-1}^{-1} \ldots \sigma_{1}^{-1}\right) a_{1, r}\left(\sigma_{1}^{-1} \ldots \sigma_{i-1}^{-1}\right) \\
& =T_{j, k} a_{i, r} .
\end{aligned}
$$

E assim, o resultado segue. Agora, vamos mostrar a relação ( $P R 7)$ : é suficiente mostrar que em $\overline{B_{n}(M)}, A_{i, r}$ comuta com $\left(a_{j, 2 g}^{-1} \ldots a_{j, 1}^{-1} T_{j, k} a_{j, 2 g} \ldots a_{j, 1}\right)$, para $1 \leq j<i \leq$ $k<n$ já que $a_{i, r}$ é uma combinação de elementos $A_{i, r}$ pelo Teorema 5.4.1. Isso será mostrado a seguir, lembrando que agora podemos usar as relações $(P R 1)-(P R 6)$ :

$$
\begin{aligned}
& A_{i, r}\left(a_{j, 2 g}^{-1} \ldots a_{j, 1}^{-1} T_{j, k} a_{j, 2 g} \ldots a_{j, 1}\right) \\
& =\left(a_{j, 2 g}^{-1} \ldots a_{j, r+1}^{-1}\right) A_{i, r}\left(a_{j, r}^{-1} \ldots a_{j, 1}^{-1}\right) T_{j, k} a_{j, 2 g} \ldots a_{j, 1} \\
& =\left(a_{j, 2 g}^{-1} \ldots a_{j, 1}^{-1}\right) T_{j, i} T_{j, i-1}^{-1} A_{i, r} T_{j, k} a_{j, 2 g} \ldots a_{j, 1} \\
& =\left(a_{j, 2 g}^{-1} \ldots a_{j, 1}^{-1}\right) T_{j, i} T_{j, i-1}^{-1} A_{i, r}\left(\sigma_{j} \ldots \sigma_{k-1}^{2} \ldots \sigma_{j}\right) a_{j, 2 g} \ldots a_{j, 1} \\
& =\left(a_{j, 2 g}^{-1} \ldots a_{j, 1}^{-1}\right) T_{j, i} T_{j, i-1}^{-1} A_{i, r}\left(\sigma_{j} \ldots \sigma_{k-1}^{2} \ldots \sigma_{1}\right) a_{1,2 g} \ldots a_{1,1}\left(\sigma_{1}^{-1} \ldots \sigma_{j-1}^{-1}\right) \\
& =\left(a_{j, 2 g}^{-1} \ldots a_{j, 1}^{-1}\right) T_{j, i} T_{j, i-1}^{-1} A_{i, r}\left(\sigma_{j} \ldots \sigma_{k-1} \sigma_{k}^{-1} \ldots \sigma_{n-1}^{-2} \ldots \sigma_{1}^{-1}\right) a_{1,1} \ldots a_{1,2 g}\left(\sigma_{1}^{-1} \ldots \sigma_{j-1}^{-1}\right) \\
& =a_{j, 2 g}^{-1} \ldots a_{j, 1}^{-1}\left(\sigma_{j} \ldots \sigma_{i-1}^{2} A_{i, r} \sigma_{i-1} \ldots \sigma_{k-1} \sigma_{k}^{-1} \ldots \sigma_{n-1}^{-2} \ldots \sigma_{1}^{-1}\right) a_{1,1} \ldots a_{1,2 g}\left(\sigma_{1}^{-1} \ldots \sigma_{j-1}^{-1}\right) \\
& =a_{j, 2 g}^{-1} \ldots a_{j, 1}^{-1}\left(\sigma_{j} \ldots \sigma_{i-1} A_{i-1, r} \sigma_{i} \ldots \sigma_{k-1} \sigma_{k}^{-1} \ldots \sigma_{n-1}^{-2} \ldots \sigma_{1}^{-1}\right) a_{1,1} \ldots a_{1,2 g}\left(\sigma_{1}^{-1} \ldots \sigma_{j-1}^{-1}\right) \\
& =a_{j, 2 g}^{-1} \ldots a_{j, 1}^{-1}\left(\sigma_{j} \ldots \sigma_{k-1} \sigma_{k}^{-1} \ldots \sigma_{n-1}^{-2} \ldots \sigma_{i}^{-1} \sigma_{i-1} \sigma_{i-2}^{-1} \ldots \sigma_{1}^{-1}\right) A_{i, r} a_{1,1} \ldots a_{1,2 g} \sigma_{1}^{-1} \ldots \sigma_{j-1}^{-1} \\
& =a_{j, 2 g}^{-1} \ldots a_{j, 1}^{-1}\left(\sigma_{j} \ldots \sigma_{k-1} \sigma_{k}^{-1} \ldots \sigma_{n-1}^{-2} \ldots \sigma_{1}^{-1}\right) a_{1,1} \ldots a_{1,2 g}\left(\sigma_{1}^{-1} \ldots \sigma_{j-1}^{-1}\right) A_{i, r} \\
& =a_{j, 2 g}^{-1} \ldots a_{j, 1}^{-1} T_{j, k}\left(\sigma_{j-1} \ldots \sigma_{1} a_{1,2 g} \ldots a_{1,1} \sigma_{1}^{-1} \ldots \sigma_{j-1}^{-1}\right) A_{i, r} \\
& =\left(a_{j, 2 g}^{-1} \ldots a_{j, 1}^{-1} T_{j, k} a_{j, 2 g} \ldots a_{j, 1}\right) A_{i, r} .
\end{aligned}
$$


Finalmente, a relação $(P R 8)$ é verificada usando alguns resultados intermediários que mostraremos a seguir. O primeiro resultado é evidente: dada a relação $(R 4)$ de $\overline{B_{n}(M)}$, temos:

$$
\begin{aligned}
A_{1,2 g} A_{2,2 g} & =\left(a_{1,1} \ldots a_{1,2 g-1}\right) A_{2,2 g} \\
& =A_{2,2 g}\left(a_{1,1} \ldots a_{1,2 g-1}\right) \\
& =A_{2,2 g} A_{1,2 g} .
\end{aligned}
$$

Mais ainda, usando a relação (6):

$$
\begin{aligned}
A_{1,2 g} A_{2,2 g} \sigma_{1} & =A_{1,2 g}\left(\sigma_{1}^{-1} A_{1,2 g} \sigma_{1}^{-1} \sigma_{1}\right) \\
& =\left(A_{1,2 g} \sigma_{1}^{-1}\right) A_{1,2 g} \\
& =\sigma_{1} A_{2,2 g} A_{1,2 g} \\
& =\sigma_{1} A_{1,2 g} A_{2,2 g} .
\end{aligned}
$$

De maneira análoga, podemos mostrar que $\left(a_{1,2 g} a_{2,2 g}\right)$ comuta com $\sigma_{1}$. O seguinte resultado é uma consequência das relações provadas acima e $(R 5)$ :

$$
\begin{aligned}
a_{1,2 g} A_{2,2 g} a_{1,2 g}^{-1} & =\left(a_{1,2 g-1}^{-1} \ldots a_{1,1}^{-1}\right) \sigma_{1}^{2} A_{2,2 g}\left(a_{1,1} \ldots a_{1,2 g-1}\right) \\
& =A_{1,2 g}^{-1} \sigma_{1}^{2} A_{2,2 g} A_{1,2 g} \\
& =A_{1,2 g}^{-1} A_{2,2 g} A_{1,2 g} \sigma_{1}^{2} \\
& =A_{2,2 g} \sigma_{1}^{2} .
\end{aligned}
$$

Portanto obtemos o seguinte resultado:

$$
a_{1,2 g} A_{2,2 g} a_{1,2 g}^{-1}=A_{2,2 g} \sigma_{1}^{2} .
$$

Agora, consideremos os fatores do lado direito de $(P R 8)$, e vemos que:

$$
\begin{aligned}
& \left(a_{i, 2 g}^{-1} \ldots a_{i, 1}^{-1}\right) T_{i, j-1} T_{i, j}^{-1}\left(a_{i, 1} \ldots a_{i, 2 g}\right) \\
& =\left(a_{i, 2 g}^{-1} \ldots a_{i, 1}^{-1}\right) \sigma_{i} \ldots \sigma_{j-2} \sigma_{j-1}^{2} \sigma_{j-2}^{-1} \ldots \sigma_{i}^{-1}\left(a_{i, 1} \ldots a_{i, 2 g}\right) \\
& =\sigma_{i-1}^{-1} \ldots \sigma_{1}^{-1}\left(a_{1,2 g}^{-1} \ldots a_{1,1}^{-1}\right) \sigma_{1} \ldots \sigma_{j-2} \sigma_{j-1}^{2} \sigma_{j-2}^{-1} \ldots \sigma_{1}^{-1}\left(a_{1,1} \ldots a_{1,2 g}\right) \sigma_{1} \ldots \sigma_{i-1} \\
& =\sigma_{i-1}^{-1} \ldots \sigma_{1}^{-1}\left(a_{1,2 g}^{-1} \ldots a_{1,1}^{-1}\right) \sigma_{j-1}^{-1} \ldots \sigma_{2}^{-1} \sigma_{1}^{-2} \sigma_{2} \ldots \sigma_{j-1}\left(a_{1,1} \ldots a_{1,2 g}\right) \sigma_{1} \ldots \sigma_{i-1} \\
& =\sigma_{i-1}^{-1} \ldots \sigma_{1}^{-1} \sigma_{j-1}^{-1} \ldots \sigma_{2}^{-1}\left(a_{1,2 g}^{-1} \ldots a_{1,1}^{-1}\right) \sigma_{1}^{-2}\left(a_{1,1} \ldots a_{1,2 g}\right) \sigma_{2} \ldots \sigma_{j-1} \sigma_{1} \ldots \sigma_{i-1}
\end{aligned}
$$




$$
\begin{aligned}
& =\left(\sigma_{i-1}^{-1} \ldots \sigma_{1}^{-1} \sigma_{j-1}^{-1} \ldots \sigma_{2}^{-1}\right) a_{1,2 g}^{-1} A_{1,2 g}^{-1} \sigma_{1}^{-2} A_{1,2 g} a_{1,2 g}\left(\sigma_{2} \ldots \sigma_{j-1} \sigma_{1} \ldots \sigma_{i-1}\right) \\
& =\left(\sigma_{i-1}^{-1} \ldots \sigma_{1}^{-1} \sigma_{j-1}^{-1} \ldots \sigma_{2}^{-1}\right) a_{1,2 g}^{-1} A_{2,2 g} \sigma_{1}^{-2} A_{2,2 g}^{-1} a_{1,2 g}\left(\sigma_{2} \ldots \sigma_{j-1} \sigma_{1} \ldots \sigma_{i-1}\right) \\
& =\left(\sigma_{i-1}^{-1} \ldots \sigma_{1}^{-1} \sigma_{j-1}^{-1} \ldots \sigma_{2}^{-1}\right) A_{2,2 g} a_{1,2 g}^{-1} \sigma_{1}^{-2} a_{1,2 g} A_{2,2 g}^{-1}\left(\sigma_{2} \ldots \sigma_{j-1} \sigma_{1} \ldots \sigma_{i-1}\right) \\
& =\left(\sigma_{i-1}^{-1} \ldots \sigma_{1}^{-1} \sigma_{j-1}^{-1} \ldots \sigma_{2}^{-1}\right) A_{2,2 g} a_{2,2 g} \sigma_{1}^{-2} a_{2,2 g}^{-1} A_{2,2 g}^{-1}\left(\sigma_{2} \ldots \sigma_{j-1} \sigma_{1} \ldots \sigma_{i-1}\right) \\
& =\left(\sigma_{i-1}^{-1} \ldots \sigma_{1}^{-1}\right) A_{j, 2 g} a_{j, 2 g}\left(\sigma_{j-1}^{-1} \ldots \sigma_{2}^{-1} \sigma_{1}^{-2} \sigma_{2} \ldots \sigma_{j-1}\right) a_{j, 2 g}^{-1} A_{j, 2 g}^{-1}\left(\sigma_{1} \ldots \sigma_{i-1}\right) \\
& =A_{j, 2 g} a_{j, 2 g}\left(\sigma_{i-1}^{-1} \ldots \sigma_{1}^{-1}\right)\left(\sigma_{j-1}^{-1} \ldots \sigma_{2}^{-1} \sigma_{1}^{-2} \sigma_{2} \ldots \sigma_{j-1}\right)\left(\sigma_{1} \ldots \sigma_{i-1}\right) a_{j, 2 g}^{-1} A_{j, 2 g}^{-1} \\
& =a_{j, 1} \ldots a_{j, 2 g}\left(\sigma_{j-1}^{-1} \ldots \sigma_{i+1}^{-1} \sigma_{i}^{-2} \sigma_{i+1} \ldots \sigma_{j-1}\right) a_{j, 2 g}^{-1} \ldots a_{j, 1}^{-1} .
\end{aligned}
$$

E isto nos dá a relação $(P R 8)$ :

$$
\begin{aligned}
& \left(\prod_{i=1}^{j-1} a_{i, 2 g}^{-1} \ldots a_{i, 1}^{-1} T_{i, j-1} T_{i, j}^{-1} a_{i, 1} \ldots a_{i, 2 g}\right) a_{j, 1} \ldots a_{j, 2 g} a_{j, 1}^{-1} \ldots a_{j, 2 g}^{-1} \\
& =\left(\prod_{i=1}^{j-1} a_{j, 1} \ldots a_{j, 2 g}\left(\sigma_{j-1}^{-1} \ldots \sigma_{i+1}^{-1} \sigma_{i}^{-2} \sigma_{i+1} \ldots \sigma_{j-1}\right) a_{j, 2 g}^{-1} \ldots a_{j, 1}^{-1}\right) a_{j, 1} \ldots a_{j, 2 g} a_{j, 1}^{-1} \ldots a_{j, 2 g}^{-1} \\
& =a_{j, 1} \ldots a_{j, 2 g}\left(\sigma_{j-1}^{-1} \ldots \sigma_{2}^{-1} \sigma_{1}^{-2} \sigma_{2}^{-1} \ldots \sigma_{j-1}^{-1}\right) a_{j, 1}^{-1} \ldots a_{j, 2 g}^{-1} \\
& =\left(\sigma_{j} \ldots \sigma_{n-1}\right) a_{n, 1} \ldots a_{n, 2 g}\left(\sigma_{n-1}^{-1} \ldots \sigma_{1}^{-2} \ldots \sigma_{n-1}^{-1}\right) a_{n, 1}^{-1} \ldots a_{n, 2 g}^{-1}\left(\sigma_{n-1} \ldots \sigma_{j}\right) \\
& =\left(\sigma_{j} \ldots \sigma_{n-1}\right)\left(\sigma_{n-1} \ldots \sigma_{j}\right) \\
& =T_{j, n} .
\end{aligned}
$$

E aqui, terminamos as relações de Tipo 1.

- Vamos encontrar agora as relações de Tipo 2. Para cada relator na apresentação de $\Sigma_{n}$, nós devemos encontrar uma palavra $U$ mencionada anteriormente. O primeiro relator em $\Sigma_{n}$ é $\delta_{i} \delta_{j} \delta_{i}^{-1} \delta_{j}^{-1}$, quando $|i-j| \geq 2$. Podemos escrevê-lo como:

$$
f\left(\sigma_{i}\right) f\left(\sigma_{j}\right) f\left(\sigma_{i}^{-1}\right) f\left(\sigma_{j}^{-1}\right) .
$$

Logo, teremos $\tilde{r}=\sigma_{i} \sigma_{j} \sigma_{i}^{-1} \sigma_{j}^{-1}$. Por outro lado, por $(R 1)$ em $\overline{B_{n}(M)}$ :

$$
\sigma_{i} \sigma_{j} \sigma_{i}^{-1} \sigma_{j}^{-1}=1,|i-j| \geq 2
$$

Vamos observar que 1 é uma palavra em $G_{A}$. Portanto, definimos $U=1$. Assim $\tilde{r}=1$ é uma relação de Tipo 2 que procuramos e mais ainda, válida em $\overline{B_{n}(M)}$. 
O segundo relator em $\Sigma_{n}$ é $\delta_{i} \delta_{i+1} \delta_{i} \delta_{i+1}^{-1} \delta_{i}^{-1} \delta_{i+1}^{-1}$. Podemos escrevê-lo como:

$$
f\left(\sigma_{i}\right) f\left(\sigma_{i+1}\right) f\left(\sigma_{i}\right) f\left(\sigma_{i+1}^{-1}\right) f\left(\sigma_{i}^{-1}\right) f\left(\sigma_{i+1}^{-1}\right), i=1, \ldots, n-2 .
$$

Logo, teremos $\tilde{r}=\sigma_{i} \sigma_{i+1} \sigma_{i} \sigma_{i+1}^{-1} \sigma_{i}^{-1} \sigma_{i+1}^{-1}$. Por outro lado, por $(R 2)$ em $\overline{B_{n}(M)}$ :

$$
\sigma_{i} \sigma_{i+1} \sigma_{i} \sigma_{i+1}^{-1} \sigma_{i}^{-1} \sigma_{i+1}^{-1}=1 ; i=1, \ldots, n-2 .
$$

Como no caso anterior, $U=1$. Logo, temos a segunda relação de Tipo 2 que procurávamos que é válida em $\overline{B_{n}(M)}$.

E para a última relação de Tipo 2, seja o relator $\delta_{i}^{2}$ de $\Sigma_{n}$ que podemos escrever como $f\left(\sigma_{i}^{2}\right)$. Logo, teremos $\tilde{r}=\sigma_{i}^{2}$. Por outro lado, por (R9) temos:

$$
\sigma_{i}^{2}=T_{i, i+1}
$$

e observemos que $T_{i, i+1}$ é uma palavra em $G_{A}$. Logo, $U=T_{i, i+1}$ e assim, temos nossa última relação de Tipo 2 que também é válida em $\overline{B_{n}(M)}$.

- Vamos terminar a prova do Teorema 5.2.2 obtendo as relações de Tipo 3 e mostrando que estas são válidas em $\overline{B_{n}(M)}$. Elas serão fáceis de deduzir e, para isto, usaremos as relações $(10),(R 1),(R 2),(R 7),(R 8)$ e $(R 9)$. Consideremos a apresentação 2 de $P B_{n}(M)$. Para cada gerador $x$ de $G_{A}$ e para cada gerador $y$ de $\Sigma_{n}$, vamos determinar tais relações:

Por (10), temos $\sigma_{i} A_{j, r} \sigma_{i}^{-1}=A_{j, r}, 1 \leq i \leq j-2$. Então, temos que a trança $\sigma_{i}$ comuta com $a_{j, r} ; j \neq i, i+1$, ou seja, $\sigma_{i} a_{j, r} \sigma_{i}^{-1}=a_{j, r}$. Vamos observar que $a_{j, r}$ é uma palavra em $G_{A}$. Logo, tomamos $V=a_{j, r}$ e assim temos as primeiras relações de Tipo 3 que procuramos.

Agora se $r$ for par, por $(R 7)$ temos $a_{i+1, r}=\sigma_{i} a_{i, r} \sigma_{i}, 1 \leq i \leq n-1$. Por $(R 9)$ temos $T_{i, i+1}=\sigma_{i}^{2}, \log \mathrm{o}, T_{i, i+1}^{-1}=\sigma_{i}^{-2}$. Portanto,

$$
\begin{aligned}
\sigma_{i} a_{i, r} \sigma_{i} \sigma_{i}^{-2} & =a_{i+1, r} \sigma_{i}^{-2} \\
& =a_{i+1, r} T_{i, i+1}^{-1}
\end{aligned}
$$

ou seja, $\sigma_{i} a_{i, r} \sigma_{i}^{-1}=a_{i+1, r} T_{i, i+1}^{-1}$. Pelo mesmo raciocínio anterior, $V=a_{i+1, r} T_{i, i+1}^{-1}$ e assim temos mais relações de Tipo 3. De modo totalmente análogo, temos 
$\sigma_{i} a_{i, r} \sigma_{i}^{-1}=T_{i, i+1} a_{i+1, r}$, se $r$ for ímpar. Com o mesmo raciocínio, encontramos as seguintes relações de Tipo 3 :

$$
\begin{aligned}
& \sigma_{i} a_{i+1, r} \sigma_{i}^{-1}=T_{i, i+1} a_{i, r}, \text { se } r \text { for par; } \\
& \sigma_{i} a_{i+1, r} \sigma_{i}^{-1}=a_{i, r} T_{i, i+1}^{-1}, \text { se } r \text { for ímpar. }
\end{aligned}
$$

Agora, para $i \neq j-1, j, k$, vamos mostrar que $\sigma_{i} T_{j, k} \sigma_{i}^{-1}=T_{j, k}$ usando $(R 1)$ e $(R 2)$. Para isso, temos que analisar os casos : $i<j \leq k, j \leq k<i$ e $j<i<k$. Os dois primeiros são simples e resolvidos apenas com $(R 1)$. Vamos fazer o último caso que requer as duas relações de tranças do disco:

$$
\begin{aligned}
\sigma_{i} T_{j, k} \sigma_{i}^{-1} & =\sigma_{i}\left(\sigma_{j} \ldots \sigma_{i-1} \sigma_{i} \sigma_{i+1} \ldots \sigma_{k-2} \sigma_{k-1}^{2} \sigma_{k-2} \ldots \sigma_{i+1} \sigma_{i} \sigma_{i-1} \ldots \sigma_{j}\right) \sigma_{i}^{-1} \\
& =\sigma_{j} \ldots \sigma_{i} \sigma_{i-1} \sigma_{i} \sigma_{i+1} \ldots \sigma_{k-2} \sigma_{k-1}^{2} \sigma_{k-2} \ldots \sigma_{i+1} \sigma_{i} \sigma_{i-1} \sigma_{i}^{-1} \ldots \sigma_{j} \\
& =\sigma_{j} \ldots \sigma_{i-1} \sigma_{i} \sigma_{i+1} \ldots \sigma_{k-2} \sigma_{k-1}^{2} \sigma_{k-2} \ldots \sigma_{i+1} \sigma_{i-1} \sigma_{i} \sigma_{i-1} \sigma_{i}^{-1} \ldots \sigma_{j} \\
& =\sigma_{j} \ldots \sigma_{i-1} \sigma_{i} \sigma_{i+1} \ldots \sigma_{k-2} \sigma_{k-1}^{2} \sigma_{k-2} \ldots \sigma_{i+1} \sigma_{i} \sigma_{i-1} \ldots \sigma_{j} \\
& =T_{j, k}
\end{aligned}
$$

Observemos que $T_{j, k}$ é uma palavra em $G_{A}$ e, portanto, temos relações de Tipo 3. E por $(R 9)$, obtemos:

$$
\begin{aligned}
T_{i, k} T_{i, i+1}^{-1} & =\left(\sigma_{i} \sigma_{i+1} \ldots \sigma_{k-2} \sigma_{k-1}^{2} \sigma_{k-2} \ldots \sigma_{i+1} \sigma_{i}\right) \sigma_{i}^{-2} \\
& =\sigma_{i}\left(\sigma_{i+1} \ldots \sigma_{k-2} \sigma_{k-1}^{2} \sigma_{k-2} \ldots \sigma_{i+1}\right) \sigma_{i}^{-1} \\
& =\sigma_{i} T_{i+1, k} \sigma_{i}^{-1}
\end{aligned}
$$

Aqui, tomamos $V=T_{i, k} T_{i, i+1}^{-1}$ e temos mais relações de Tipo 3. Também:

$$
\begin{aligned}
\sigma_{i} T_{i, k} \sigma_{i}^{-1} & =\sigma_{i}\left(\sigma_{i} \sigma_{i+1} \ldots \sigma_{k-2} \sigma_{k-1}^{2} \sigma_{k-2} \ldots \sigma_{i+1} \sigma_{i}\right) \sigma_{i}^{-1} \\
& =\sigma_{i} \sigma_{i}\left(\sigma_{i+1} \ldots \sigma_{k-2} \sigma_{k-1}^{2} \sigma_{k-2} \ldots \sigma_{i+1}\right) \\
& =\sigma_{i}^{2}\left(\sigma_{i+1} \ldots \sigma_{k-2} \sigma_{k-1}^{2} \sigma_{k-2} \ldots \sigma_{i+1}\right) \\
& =T_{i, i+1} T_{i+1, k} .
\end{aligned}
$$

Nesta relação, temos $V=T_{i, i+1} T_{i+1, k}$. Vamos agora para as últimas relações de Tipo 3, dadas por: 


$$
\begin{aligned}
\sigma_{i}^{-1} T_{j, i-1} T_{j, i}^{-1} T_{j, i+1} \sigma_{i} & =\sigma_{i}^{-1}\left(\sigma_{j} \ldots \sigma_{i-3} \sigma_{i-2}^{2} \sigma_{i-3} \ldots \sigma_{j}\right)\left(\sigma_{j}^{-1} \ldots \sigma_{i-2}^{-1} \sigma_{i-1}^{-2} \sigma_{i-2}^{-1} \ldots \sigma_{j}^{-1}\right) \\
& \left(\sigma_{j} \ldots \sigma_{i-1} \sigma_{i}^{2} \sigma_{i-1} \ldots \sigma_{j}\right) \\
& =\sigma_{i}^{-1}\left(\sigma_{j} \ldots \sigma_{i-3} \sigma_{i-2}^{-2}\right)\left(\sigma_{i-1}^{-1}\right)\left(\sigma_{i}^{2} \sigma_{i-1} \ldots \sigma_{j}\right) \sigma_{i} \\
& =\left(\sigma_{j} \ldots \sigma_{i-3} \sigma_{i-2}\right)\left(\sigma_{i}^{-1} \ldots \sigma_{i-1}^{-1} \sigma_{i} \sigma_{i} \sigma_{i-1} \sigma_{i}\left(\sigma_{i-2} \sigma_{i-3} \ldots \sigma_{j}\right)\right. \\
& =T_{j, i}
\end{aligned}
$$

Portanto, temos $\sigma_{i} T_{j, i} \sigma_{i}^{-1}=T_{j, i-1} T_{j, i}^{-1} T_{j, i+1}$ e terminamos as relações de Tipo 3. Como todas as relações do Tipo 1,2 e 3 são válidas em $\overline{B_{n}(M)}$ então segue que $\psi$ é um isomorfismo e portanto, o Teorema 5.2.2 fica provado.

\subsection{O grupo de tranças de uma superfície não orientável}

Nesta seção vamos demonstrar o Teorema 5.2.3 usando o mesmo método do caso orientável. Então, suponhamos $M$ uma superfície fechada, não orientável de gênero $g \geq 2$.

Passo 1. Vamos denotar por $\overline{P B_{n}(M)}$ o grupo definido pela apresentação a seguir:

\section{Apresentação 3:}

Geradores: $\left\{a_{i, r} ; 1 \leq i \leq n, 1 \leq r \leq g\right\} \cup\left\{T_{j, k} ; 1 \leq j<k \leq n\right\}$.

\section{Relações:}

(Pr1) $a_{n, 1}^{2} a_{n, 2}^{2} \ldots a_{n, g}^{2}=\prod_{i=1}^{n-1} T_{i, n-1}^{-1} T_{i, n}$.

(Pr2) $a_{i, r} A_{j, s}=A_{j, s} a_{i, r}$ $1 \leq i<j \leq n ; 1 \leq r, s \leq g ; r \neq s$.

(Pr4) $T_{i, j} T_{k, l}=T_{k, l} T_{i, j}$ $1 \leq i<j<k<l \leq n$ ou $1 \leq i<k<l \leq j \leq n$.

(Pr5) $T_{k, l} T_{i, j} T_{k, l}^{-1}=T_{i, k-1} T_{i, k}^{-1} T_{i, j} T_{i, l}^{-1} T_{i, k} T_{i, k-1}^{-1} T_{i, l}$ $1 \leq i<k \leq j<l \leq n$.

$(\operatorname{Pr} 6) a_{i, r} T_{j, k}=T_{j, k} a_{i, r}$ $1 \leq i<j<k \leq n$ ou $1 \leq j<k<i \leq n ; 1 \leq r \leq g$.

$$
a_{i, r}\left(a_{j, g}^{-2} \ldots a_{j, 1}^{-2} T_{j, k}\right)=\left(a_{j, g}^{-2} \ldots a_{j, 1}^{-2} T_{j, k}\right) a_{i, r} \quad 1 \leq j<i \leq k \leq n
$$

(Pr8) $T_{j, n}=a_{j, 1}^{2} \ldots a_{j, g}^{2}\left(\prod_{i=1}^{j-1} T_{j-i, j}^{-1} T_{j-i, j-1}\right)$. 
Onde

$$
A_{j, r}=a_{j, 1}^{2} \ldots a_{j, r-1}^{2} a_{j, r}^{-1} a_{j, r-1}^{-2} \ldots a_{j, 1}^{-2}
$$

Da mesma forma que na seção anterior, faremos uso de um lema pra extrairmos outra apresentação de $\overline{P B_{n}(M)}$ que é dado a seguir:

Lema 5.5.1. Seja F um grupo livre finitamente gerado por $\left\{x_{1}, \ldots, x_{g}\right\}$. Seja $X_{r}=$ $x_{1}^{2} \ldots x_{r-1}^{2} x_{r}^{-1} x_{r-1}^{-2} \ldots x_{1}^{-2}$. Então $\left\{X_{1}, \ldots, X_{g}\right\}$ é um sistema livre de geradores de $F$.

Demonstração: A demosntração é bem simples e a fórmula da mudança de geradores é dada por:

$$
x_{k}=\left(X_{1}^{2} X_{2}^{2} \ldots X_{k-1}^{2} X_{k}^{-1} X_{k-1}^{-2} \ldots X_{2}^{-2} X_{1}^{-2}\right) .
$$

Observemos que $\overline{P B_{n}(M)}$ tem no seu conjunto de geradores o fator $\left\{a_{i, r} ; 1 \leq i \leq\right.$ $n, 1 \leq r \leq g\}$. E também, a definição de $A_{j, r}=a_{j, 1}^{2} \ldots a_{j, r-1}^{2} a_{j, r}^{-1} a_{j, r-1}^{-2} \ldots a_{j, 1}^{-2}$ é exatamente a definição de $X_{r}$ que temos no lema anterior. Logo, temos:

\section{Apresentação 4:}

Geradores: $\left\{A_{i, r} ; 1 \leq i \leq n, 1 \leq r \leq g\right\} \cup\left\{T_{j, k} ; 1 \leq j<k \leq n\right\}$.

Relações: as mesmas da apresentação 3, onde:

$$
a_{i, r}=A_{i, 1}^{2} \ldots A_{i, r-1}^{2} A_{i, r}^{-1} A_{i, r-1}^{-2} \ldots A_{i, 1}^{-2} .
$$

Usando a apresentação 3 , vamos definir $\tilde{\varphi}: \overline{P B_{n}(M)} \rightarrow P B_{n}(M)$ da maneira mais natural possível:

$$
\begin{aligned}
\tilde{\varphi} & : \overline{P B_{n}(M)} \rightarrow P B_{n}(M) \\
& a_{i, r} \longmapsto a_{i, r}, \quad 1 \leq i \leq n, 1 \leq r \leq g \\
& T_{i, j} \longmapsto T_{i, j}, \quad 1 \leq i<j \leq n .
\end{aligned}
$$

Por abuso de notação, continuaremos denotando por $a_{i, r}$ e $T_{i, j}$ as tranças que serão as imagens de $a_{i, r}$ e $T_{i, j}$ respectivamente sobre o homomorfismo $\tilde{\varphi}$. Essas tranças são definidas como segue: para cada $i, j$ com $1 \leq i \leq j \leq n$, a trança $T_{i, j}$ é definida exatamente como fizemos na seção anterior. Para cada $i, r$ com $1 \leq i \leq n$ e $1 \leq r \leq g$, a trança $a_{i, r}$ será 
representada pela $i$-ésima corda passando pela $r$-ésima parede, como na figura a seguir. Definimos também os caminhos $e_{i}, i=1, \ldots, n$ os quais vão do ponto $P_{i}$ para o ponto final de $e$ :
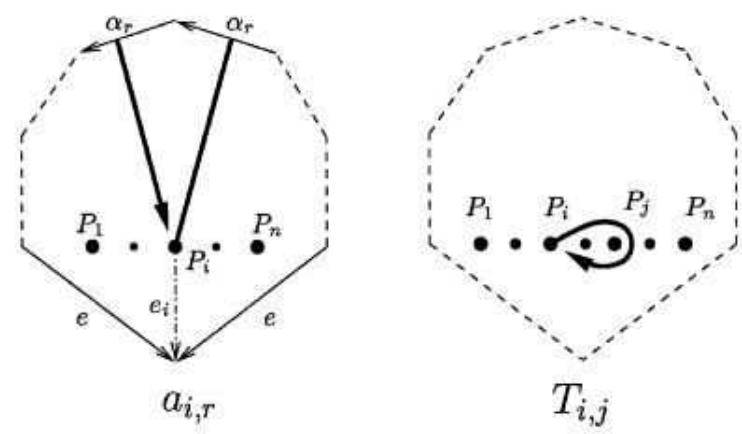

Figura 5.27: Os geradores de $P B_{n}(M)$.

Dado $i \in\{1, \ldots, n\}$, vamos denotar por $s_{i, r}$ a $i$-ésima corda de $a_{i, r}$. E, pelo mesmo processo feito para o $P_{1}$-polígono na seção 5.2.2 definimos o $P_{i}$-polígono: vamos "recortar"

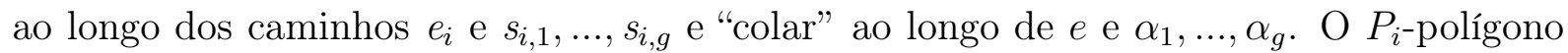
resultante é rotulado pelos caminhos:

$$
s_{i, 1}, s_{i, 1}, s_{i, 2}, s_{i, 2}, \ldots, s_{i, g}, s_{i, g}, e_{i}, e_{i}^{-1}
$$

no sentido horário.

Agora podemos repetir o processo da seção 5.2.2 para ver que, para $1 \leq i<j$, a trança $A_{j, r}=a_{j, 1}^{2} \ldots a_{j, r-1}^{2} a_{j, r}^{-1} a_{j, r-1}^{-2} \ldots a_{j, 1}^{-2}$ pode ser representada no $P_{i}$-polígono da figura a seguir:

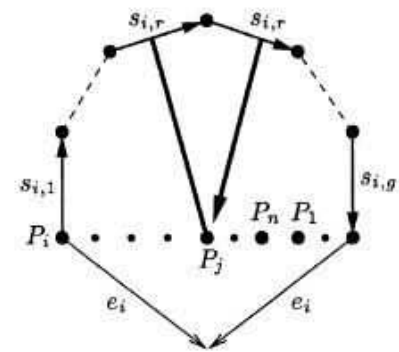

Figura 5.28: A trança $A_{j, r}$ no $P_{i}$-polígono $(\mathrm{i}<\mathrm{j})$.

Agora, mostremos que $\tilde{\varphi}$ é um homomorfismo. Para isto, temos que mostrar que as relações de $\overline{P B_{n}(M)}$ são válidas em $P B_{n}(M)$. De fato:

- As relações $(\operatorname{Pr} 4)$ e $(\operatorname{Pr} 5)$ estão em $P B_{n}(M)$ já que estas tranças podem ser vistas 
como tranças puras no disco. A representação que deixa claro que a relação $(\operatorname{Pr} 4)$ é válida em $P B_{n}(M)$ segue abaixo:

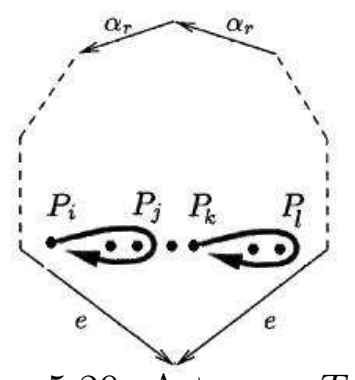

Figura 5.29: A trança $T_{i, j} T_{k, l}$.

Para a relação (Pr5) também é simples: isolamos todos os termos desta relação e mostramos que este produto é equivalente à trança trivial. Ela é válida em $P B_{n}(M)$ já que podemos vê-la como uma trança pura no disco.

- A relação (Pr6) também é óbvia graças à representação seguinte:

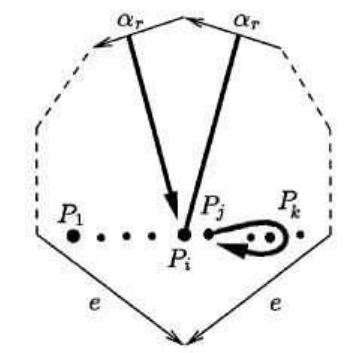

Figura 5.30: A trança $a_{i, r} T_{j, k}$.

- Podemos mostrar que as relações $(\operatorname{Pr} 1),(\operatorname{Pr} 2)$ e $(\operatorname{Pr} 3)$ são válidas em $\operatorname{P} B_{n}(M)$ da mesma forma que mostramos as relações $(r 1),(r 2)$ e $(r 3)$ respectivamente. De fato, para a relação $(\operatorname{Pr} 1)$ temos que o produto de tranças $\prod_{i=1}^{n-1} T_{i, n-1}^{-1} T_{i, n}$ é equivalente, via $P_{n}$-polígono, à trança da figura seguir:

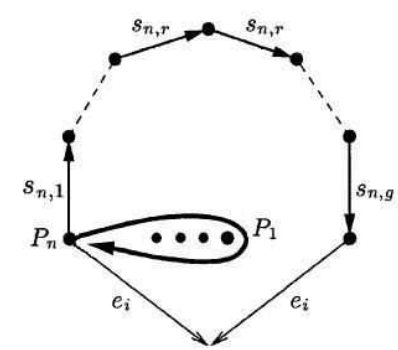

Figura 5.31: A trança $a_{n, 1}^{2} \ldots a_{n, g}^{2}$. 
que por sua vez é equivalente à trança $a_{n, 1}^{2} a_{n, 2}^{2} \ldots a_{n, g}^{2}$.

Portanto, a relação $(\operatorname{Pr} 1)$ é satisfeita em $P B_{n}(M)$. Agora, a validade da relação (Pr2) em $P B_{n}(M)$ é dada com a ajuda da representação do $P_{i}$-polígono de $A_{j, s}$ $(i<j)$ da Figura 5.28. Também, a relação $(\operatorname{Pr} 3)$ é válida em $P B_{n}(M)$ já que seu $P_{i}$-polígono é feito de modo totalmente análogo à Figura 5.14.

- Vamos considerar o $P_{j}$-polígono de $\left(a_{j, g}^{-2} \ldots a_{j, 1}^{-2} T_{j, k}\right)$ para a verificação da relação $(P R 7)$ :

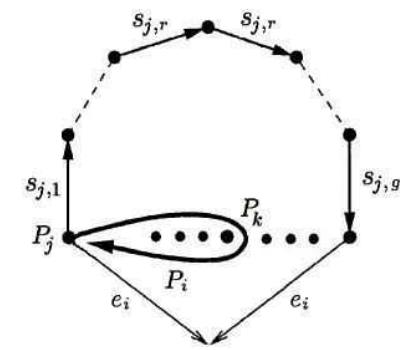

Figura 5.32: $\left(a_{j, g}^{-2} \ldots a_{j, 1}^{-2} T_{j, k}\right)$

Desta forma, $a_{i, r}$ comuta com $\left(a_{j, g}^{-2} \ldots a_{j, 1}^{-2} T_{j, k}\right)$, para cada $1 \leq j<i \leq k \leq n$. E assim, temos que a relação $(P R 7)$ é válida em $P B_{n}(M)$.

- Finalmente, para verificarmos que a relação $(\operatorname{Pr} 8)$ é válida em $P B_{n}(M)$ vamos usar o raciocínio do caso orientável e obtemos a seguinte trança:

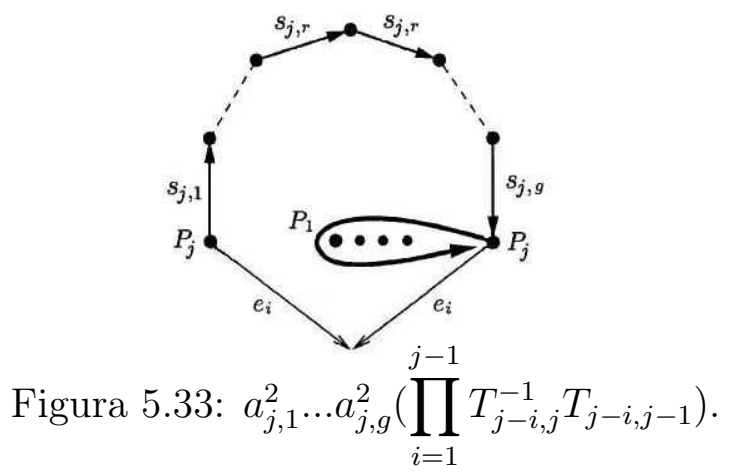

E assim, temos que a trança acima é equivalente a $T_{j, n}$.

Pelas considerações acima, mostramos que $\tilde{\varphi}$ é um homomorfismo bem definido, e assim terminamos o passo 1.

Passo 2. Vamos mostrar por indução sobre $n$ que $\tilde{\varphi}$ é um isomorfismo. 
Consideremos:

$$
\begin{aligned}
\tilde{\varphi}: & \overline{P B_{n}(M)} \rightarrow P B_{n}(M) \\
& a_{i, r} \longmapsto a_{i, r}, 1 \leq i \leq n, 1 \leq r \leq g \\
& T_{j, k} \longmapsto T_{j, k}, \quad 1 \leq j<k \leq n .
\end{aligned}
$$

Se $n=1$ então a apresentação de $\overline{P B_{1}(M)}$ reduz-se à:

$$
\overline{P B_{1}(M)}=<\left\{a_{1,1}, \ldots, a_{1, g}\right\} ; a_{1,1}^{2} \ldots a_{1, g}^{2}=1>
$$

Vamos observar que a apresentação acima é a mesma apresentação de $\pi_{1}(M)$ que estudamos no capítulo 3 e, na primeira seção deste capítulo vimos que $\pi_{1}(M)=P B_{1}(M)$. Além disso, desde que $n=1$ temos $\tilde{\varphi}\left(a_{1, r}\right)=a_{1, r}=s_{1, r}$, para $r=1, \ldots, g$. Logo, $\tilde{\varphi}$ é um homomorfismo de grupos que possuem as mesmas apresentações e que leva os geradores de $\overline{P B_{1}(M)}$ nos geradores de $P B_{1}(M)$. Portanto, $\overline{P B_{1}(M)} \stackrel{\tilde{\varphi}}{\simeq} P B_{1}(M)$.

Agora para a hipótese de indução suponhamos que $\overline{P B_{n-1}(M)} \stackrel{\tilde{\varphi}}{\simeq} P B_{n-1}(M)$. Logo, $P B_{n-1}(M)$ admite as apresentações 3 e 4 como apresentações:

\section{Apresentação 3}

Geradores: $\left\{a_{i, r} ; 1 \leq r \leq g\right\} \cup\left\{T_{i, j} ; 1 \leq j<k \leq n-1\right\}$.

\section{Relações:}

$$
\begin{aligned}
& \text { (Pr1) } a_{n-1,1}^{2} \ldots a_{n-1, g}^{2}=\prod_{i=1}^{n-2} T_{i, n-2}^{-1} T_{i, n-1} . \\
& \text { (Pr2) } a_{i, r} A_{j, s}=A_{j, s} a_{i, r} \\
& 1 \leq i<j \leq n-1 ; 1 \leq r, s \leq g ; r \neq s . \\
& \text { (Pr3) }\left(a_{i, 1}^{2} \ldots a_{i, r-1}^{2} a_{i, r}\right) A_{j, r}\left(a_{i, r}^{-1} a_{i, r-1}^{-2} \ldots a_{i, 1}^{-2}\right) A_{j, r}^{-1}=T_{i, j} T_{i, j-1}^{-1} \\
& 1 \leq i<j \leq n-1 ; 1 \leq r \leq g . \\
& \text { (Pr4) } T_{i, j} T_{k, l}=T_{k, l} T_{i, j} \quad 1 \leq i<j<k<l \leq n-1 \text { ou } 1 \leq i<k<l \leq j \leq n-1 . \\
& \text { (Pr5) } T_{k, l} T_{i, j} T_{k, l}^{-1}=T_{i, k-1} T_{i, k}^{-1} T_{i, j} T_{i, l}^{-1} T_{i, k} T_{i, k-1}^{-1} T_{i, l} \\
& 1 \leq i<k \leq j<l \leq n-1 \\
& \text { (Pr6) } a_{i, r} T_{j, k}=T_{j, k} a_{i, r} \quad 1 \leq i<j<k \leq n-1 \text { ou } 1 \leq j<k<i \leq n-1 ; 1 \leq r \leq g . \\
& (\operatorname{Pr} 7) a_{i, r}\left(a_{j, g}^{-2} \ldots a_{j, 1}^{-2} T_{j, k}\right)=\left(a_{j, g}^{-2} \ldots a_{j, 1}^{-2} T_{j, k}\right) a_{i, r} \\
& 1 \leq j<i \leq k \leq n-1 \text {. } \\
& \text { (Pr8) } T_{j, n-1}=a_{j, 1}^{2} \ldots a_{j, g}^{2}\left(\prod_{i=1}^{j-1} T_{j-i, j}^{-1} T_{j-i, j-1}\right) .
\end{aligned}
$$


Onde:

$$
A_{j, s}=a_{j, 1}^{2} \ldots a_{j, s-1}^{2} a_{j, s}^{-1} a_{j, s-1}^{-2} \ldots a_{j, 1}^{-2} .
$$

\section{Apresentação 4}

Geradores: $\left\{A_{i, r} ; 1 \leq i \leq n-1,1 \leq r \leq g\right\} \cup\left\{T_{i, j} ; 1 \leq j<k \leq n-1\right\}$.

Relações: as mesmas da apresentação 3, onde:

$$
a_{i, r}=A_{i, 1}^{2} \ldots A_{i, r-1}^{2} A_{i, r}^{-1} A_{i, r-1}^{-2} \ldots A_{i, 1}^{-2} .
$$

Agora, vamos tomar a sequência exata (2):

$$
1 \longrightarrow \pi_{1}\left(M \backslash \mathcal{P}^{\prime}, P_{1}\right) \stackrel{u}{\longrightarrow} P B_{n}(M, \mathcal{P}) \stackrel{\nu}{\longrightarrow} P B_{n-1}\left(M, \mathcal{P}^{\prime}\right) \longrightarrow 1
$$

onde $u(\gamma)=\left(\gamma, e P_{2}, \ldots, e P_{n}\right)$, para cada $\gamma \in \pi_{1}\left(M \backslash \mathcal{P}^{\prime}, P_{1}\right)$ e $\nu(\Gamma)=\left(\gamma_{2}, \ldots, \gamma_{n}\right)$, para cada $\Gamma \in P B_{n}(M)$.

Em ordem para aplicar o Lema 5.3.1, temos que conhecer as apresentações dos grupos à esquerda e à direita de $P B_{n}(M, \mathcal{P})$ na sequência exata (2). Para o grupo à esquerda, fazemos a seguinte afirmação:

Afirmação 2: O grupo fundamental $\pi_{1}\left(M \backslash \mathcal{P}^{\prime}, P_{1}\right)$ é livre e admite a seguinte apresentação: $<\left\{s_{1,1}, \ldots, s_{1, g}, t_{1,2}, \ldots, t_{1, n-1}\right\} ;->$. De fato, vamos considerar os abertos conexos por caminhos $U, V$ e $U \cap V$ dados na figura abaixo:
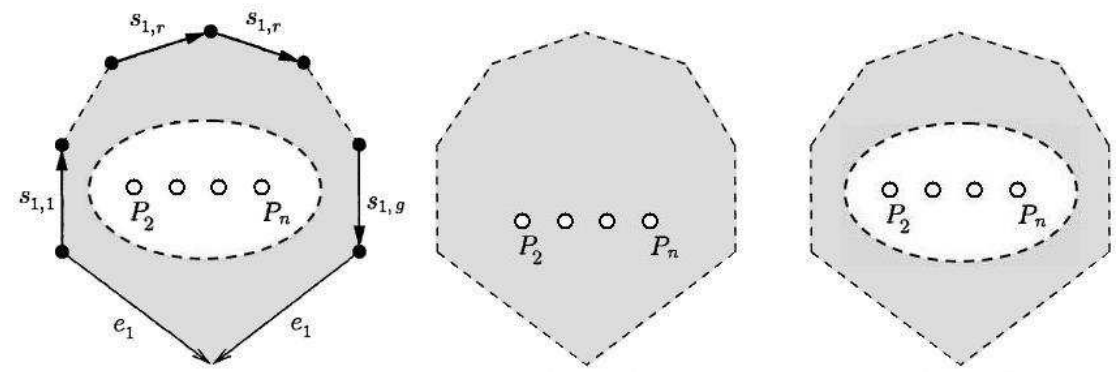

Figura 5.34: Os abertos $U, V$ e $U \cap V$.

Agora, aplicando o Teorema 3.1 .8 (versão 3 do Teorema de Seifert-Van Kampen) juntamente com o Teorema 3.1.5, temos:

$$
\pi_{1}\left(M \backslash \mathcal{P}^{\prime}, P_{1}\right) \simeq \frac{\pi_{1}\left(U, P_{1}\right) * \pi_{1}\left(V, P_{1}\right)}{N}
$$


O aberto $U$ possui o mesmo tipo de homotopia da soma conexa de $g$ planos projetivos menos um ponto. Logo, possui o mesmo tipo de homotopia da rosácea de $g$-pétalas. Portanto:

$$
\pi_{1}\left(U, P_{1}\right)=<\left\{s_{1,1}, \ldots, s_{1, g}\right\} ;->
$$

Também, o aberto $V$ possui o mesmo tipo de homotopia da rosácea de $(n-1)$-pétalas. Portanto:

$$
\pi_{1}\left(V, P_{1}\right)=<\left\{t_{1,2}, \ldots, t_{1, n}\right\} ;->
$$

Agora, vamos determinar $N$. Seja $[\gamma]$ um gerador de $\pi_{1}\left(U \cap V, P_{1}\right)$. Logo, $\left(i_{1}\right)_{*}[\gamma]=$ $\left[s_{1,1}^{2} \ldots s_{1, g}^{2}\right]$ e $\left(i_{2}\right)_{*}[\gamma]=\left[t_{1, n}\right]$. Mas da mesma forma que no caso orientável, pelo $P_{1}$-polígono de $t_{1, n}$ temos:

$$
t_{1, n}=s_{1,1}^{2} \ldots s_{1, g}^{2}
$$

Logo, $\left(\left(i_{1}\right)_{*}[\gamma]\right) *\left(\left(i_{2}\right)_{*}[\gamma]\right)^{-1}=1$, ou seja, $N=\left[\left(\left(i_{1}\right)_{*}[\gamma]\right) *\left(\left(i_{2}\right)_{*}[\gamma]\right)^{-1}\right]=1$. Desde que $U \cap V$ tem o mesmo tipo de homotopia de $S^{1}$ e $\pi_{1}\left(S^{1}\right)$ é cíclico, é suficiente fazer o cálculo acima apenas para o gerador.

Assim, $\pi_{1}\left(M \backslash \mathcal{P}^{\prime}, P_{1}\right)=<\left\{s_{1,1}, \ldots, s_{1, g}, t_{1,2}, \ldots, t_{1, n}\right\} ;->$. Mas observemos que $t_{1, n}$ é escrito como combinação dos elementos do conjunto $\left\{s_{1,1}, \ldots, s_{1, g}\right\}$ e assim podemos remover $t_{1, n}$ dos geradores de $\pi_{1}\left(M \backslash \mathcal{P}^{\prime}, P_{1}\right)$. Portanto,

$$
\pi_{1}\left(M \backslash \mathcal{P}^{\prime}, P_{1}\right)=<\left\{s_{1,1}, \ldots, s_{1, g}, t_{1,2}, \ldots, t_{1, n-1}\right\} ;->
$$

e desta forma a afirmação fica provada.

Será bom para os nossos proprósitos incluir $t_{1, n}$ entre os geradores. Então, vamos incluir uma única relação, a saber, a relação que extraímos do $P_{1}$-polígono. Desta forma, vamos obter a seguinte apresentação:

$$
\pi_{1}\left(M \backslash \mathcal{P}^{\prime}, P_{1}\right)=<\left\{s_{1,1}, \ldots, s_{1, g}, t_{1,2}, \ldots, t_{1, n}\right\} ; t_{1, n}=s_{1,1}^{2} \ldots s_{1, g}^{2}>
$$

Logo, temos uma apresentação para o grupo da esquerda na sequência exata (2).

Para o grupo $P B_{n-1}(M)$ temos duas apresentações garantidas pela hipótese de indução. Agora, utilizaremos a apresentação 4. Desta forma, já podemos aplicar o Lema 5.3.1 para conseguirmos uma apresentação para $P B_{n}(M, \mathcal{P})$.

Vamos observar que $\nu\left(a_{i, r}\right)=a_{i-1, r}$, para $i=2, \ldots, n$, então, desde que $\nu$ é homomorfismo, temos $\nu\left(A_{i, r}\right)=A_{i-1, r}$, para $i=2, \ldots, n$. Também, $\nu\left(T_{i, j}\right)=T_{i-1, j-1}$, para 
$2 \leq i \leq j \leq n$. Desta forma, conhecemos as pré- imagens por $\nu$ dos geradores de $P B_{n-1}\left(M, \mathcal{P}^{\prime}\right)$. Vamos observar também que $u\left(s_{1, r}\right)=a_{1, r}$ e $u\left(t_{1, j}\right)=T_{1, j}$ para todos os $r$ e $j$ possíveis. Então, obtemos imediatamente o seguinte conjunto de geradores para $P B_{n}(M ; \mathcal{P}):$

$$
\left\{a_{1, r} ; 1 \leq r \leq g\right\} \cup\left\{A_{i, r} ; 2 \leq i \leq n, 1 \leq r \leq g\right\} \cup\left\{T_{j, k} ; 1 \leq j<k \leq n\right\} .
$$

Ainda podemos simplificar este conjunto de geradores aplicando o Lema 5.4.1:

$$
\left\{a_{i, r} ; 1 \leq i \leq n, 1 \leq r \leq g\right\} \cup\left\{T_{j, k} ; 1 \leq j<k \leq n\right\}
$$

o qual é a imagem por $\tilde{\varphi}$ do conjunto de geradores de $\overline{P B_{n}(M)}$. Em particular, $\tilde{\varphi}$ é sobrejetor.

Agora, vamos provar que $\tilde{\varphi}$ é um isomorfismo através do seguinte procedimento: vamos considerar as relações em $P B_{n}(M)$ que extrairemos do Lema 5.3.1, e provar que as mesmas relações, quando consideradas em $\overline{P B_{n}(M)}$ aindam continuam válidas. Primeiro, vamos denotar por $G_{A}$ o conjunto de geradores de $\pi_{1}\left(M \backslash \mathcal{P}^{\prime}, P_{1}\right)$, e por $G$ o conjunto de geradores de $\overline{P B_{n}(M)}$. Consideremos a única relação na apresentação de $\pi_{1}\left(M \backslash \mathcal{P}^{\prime}, P_{1}\right)$, a qual consideramos uma relação em $P B_{n}(M)$ via o homomorfismo $u$. Esta será única relação de Tipo 1 na apresentação de $P B_{n}(M)$. O procedimento começa mostrando que esta relação é válida quando considerada em $\overline{P B_{n}(M)}$, isto é, temos uma relação em $\overline{P B_{n}(M)}$ a qual é aplicada por $\tilde{\varphi}$ na única relação na apresentação de $\pi_{1}\left(M \backslash \mathcal{P}^{\prime}, P_{1}\right)$.

Depois, para o próximo relator $r$ de $P B_{n-1}(M)$, vamos considerar a pré-imagem "canônica" por $\nu$ de $r$, denotada por $\tilde{r}$, no sentido do Lema 5.3.1. Desde que $P B_{n}(M)$ e $\overline{P B_{n}(M)}$ possuem "os mesmos" geradores via o homomorfismo $\tilde{\varphi}$, podemos considerar também $\tilde{r}$ como uma palavra em $G$. Agora, encontramos uma palavra $U$ em $G$ tal que a igualdade $\tilde{r}=U$ é válida em $\overline{P B_{n}(M)}$, e tal que $\tilde{\varphi}(U)$ é uma palavra em $G_{A}$. Isto nos dará as relações de Tipo $2 \tilde{r}=\tilde{\varphi}(U)$ na apresentação de $P B_{n}(M)$.

Finalmente, para cada $x \in G_{A}$ e cada gerador $y$ de $P B_{n-1}(M)$, encontramos uma palavra $V$ em $G$ tal que a igualdade $\tilde{y} x \tilde{y}^{-1}=V$ é válida em $\overline{P B_{n}(M)}$, onde $\tilde{y}$ é a préimagem canônica por $\nu$ de $y$, e tal que $\tilde{\varphi}(V)$ é uma palavra em $G_{A}$. Isto nos dará as relações de Tipo 3 na apresentação de $P B_{n}(M)$.

Deste modo, teremos encontrado todas as relações de Tipo 1,2 e 3 do Lema 5.3.1 e, portanto, uma apresentação de $P B_{n}(M)$, e, ao mesmo tempo,teremos mostrado que $\tilde{\varphi}$ é injetor e, consequentemente, $\tilde{\varphi}$ é um isomorfismo. 
Vamos começar com o procedimento:

- Consideremos a única relação na apresentação de $\pi_{1}\left(M \backslash \mathcal{P}^{\prime}, P_{1}\right)$ :

$$
s_{1,1}^{2} \ldots s_{1, g}^{2}=t_{1, n}
$$

Aplicando o homomorfismo $u$, temos:

$$
a_{1,1}^{2} \ldots a_{1, g}^{2}=T_{1, n}
$$

que corresponde à relação $(\operatorname{Pr} 8)$ de $\overline{P B_{n}(M)}$, para $j=1$. Então a relação é válida neste grupo. E mais, é a única relação de Tipo 1 de $P B_{n}(M)$.

- As relações de Tipo 2 são fáceis de encontrar:

Vamos começar com o relator de $(\operatorname{Pr} 1)$ de $P B_{n-1}(M)$ :

$$
a_{n-1,1}^{2} \ldots a_{n-1, g}^{2}\left(\prod_{i=1}^{n-2} T_{i, n-2}^{-1} T_{i, n-1}\right)^{-1}
$$

que podemos escrever como:

$$
\nu\left(a_{n, 1}^{2}\right) \ldots \nu\left(a_{n, g}^{2}\right) \nu\left(\left(\prod_{i=1}^{n-2} T_{i+1, n-1}^{-1} T_{i+1, n}\right)^{-1}\right) .
$$

Assim, pela pré-imagem de $\nu$, temos $\tilde{r} \in P B_{n}(M)$ :

$$
\tilde{r}=a_{n, 1}^{2} \ldots a_{n, g}^{2}\left(\prod_{i=1}^{n-2} T_{i+1, n-1}^{-1} T_{i+1, n}\right)^{-1}
$$

Para nossa conveniência vamos fazer uma pequena mudança de índices no produtório em $\tilde{r}$ :

$$
\tilde{r}=a_{n, 1}^{2} \ldots a_{n, g}^{2}\left(\prod_{i=2}^{n-1} T_{i, n-1}^{-1} T_{i, n}\right)^{-1} .
$$

Por outro lado, pela relação $(\operatorname{Pr} 1)$ de $\overline{P B_{n}(M)}$, temos:

$$
a_{n, 1}^{2} \ldots a_{n, g}^{2}=\prod_{i=1}^{n-1} T_{i, n-1}^{-1} T_{i, n}
$$


que pode ser escrito como:

$$
a_{n, 1}^{2} \ldots a_{n, g}^{2}\left(\prod_{i=2}^{n-1} T_{i, n-1}^{-1} T_{i, n}\right)^{-1}=T_{1, n-1}^{-1} T_{1, n} .
$$

Neste caso, temos $U=T_{1, n-1}^{-1} T_{1, n}$.

Notemos que $\tilde{r}$ pode ser visto como uma palavra em $G$ já que $P B_{n}(M)$ e $\overline{P B_{n}(M)}$ possuem os "mesmos" geradores e $\tilde{r}$ aparece na relação $(\operatorname{Pr} 1)$ de $P B_{n}(M)$. Logo, $\tilde{r}=U$, ou seja, $\tilde{r}=\tilde{\varphi}(U)$. Mas $\tilde{\varphi}(U)=\tilde{\varphi}\left(T_{1, n-1}^{-1} T_{1, n}\right)=T_{1, n-1}^{-1} T_{1, n}=u\left(t_{1, n-1}^{-1} t_{1, n}\right)$, ou seja, uma palavra em $G_{A}$. Portanto,

$$
a_{n, 1}^{2} \ldots a_{n, g}^{2}\left(\prod_{i=2}^{n-1} T_{i, n-1}^{-1} T_{i, n}\right)^{-1}=T_{1, n-1}^{-1} T_{1, n}
$$

é a relação de Tipo 2 que estávamos procurando. Além disso, esta relação é válida em $\overline{P B_{n}(M)}$ pois $\tilde{r}=\tilde{\varphi}(U)$ implica na relação $(\operatorname{Pr} 1)$ da apresentação de $\overline{P B_{n}(M)}$. Agora, seja o relator de $(\operatorname{Pr} 2)$ :

$$
a_{i, r} A_{j, s} a_{i, r}^{-1} A_{j, s}^{-1}
$$

de $P B_{n-1}(M)$. Para nossa conveniência, vamos fazer a mudança de índices:

$$
a_{i-1, r} A_{j-1, s} a_{i-1, r}^{-1} A_{j-1, s}^{-1}, \quad 0 \leq i-1 \leq j-1 \leq n-2,1 \leq r, s \leq g, r \neq s .
$$

que podemos escrever como:

$$
\nu\left(a_{i, r}\right) \nu\left(A_{j, s}\right) \nu\left(a_{i, r}^{-1}\right) \nu\left(A_{j, s}^{-1}\right), \quad i \geq 2 .
$$

Assim, pela pré-imagem de $\nu$, temos $\tilde{r} \in P B_{n}(M)$ :

$$
\tilde{r}=a_{i, r} A_{j, s} a_{i, r}^{-1} A_{j, s}^{-1}, \quad i \geq 2 .
$$

Por outro lado, pela relação $(\operatorname{Pr} 2)$ de $\overline{P B_{n}(M)}$, temos:

$$
a_{i, r} A_{j, s} a_{i, r}^{-1} A_{j, s}^{-1}=1, \quad i \geq 2
$$


Neste caso, temos $U=1$.

Notemos que $\tilde{r}$ pode ser visto como uma palavra em $G$ já que $P B_{n}(M)$ e $\overline{P B_{n}(M)}$ "possuem" os mesmos geradores e $\tilde{r}$ aparece na relação $(\operatorname{Pr} 2)$ de $P B_{n}(M)$. Logo, $\tilde{r}=U$, ou seja, $\tilde{r}=\tilde{\varphi}(U)$. Mas $\tilde{\varphi}(U)=\tilde{\varphi}(1)=1$, ou seja, uma palavra em $G_{A}$. Portanto,

$$
a_{i, r} A_{j, s} a_{i, r}^{-1} A_{j, s}^{-1}=1, \quad i \geq 2
$$

é uma relação de Tipo 2 que estávamos procurando. Além disso, esta relação é válida em $\overline{P B_{n}(M)}$ pois $\tilde{r}=\tilde{\varphi}(U)$ implica na relação $(\operatorname{Pr} 2)$ da apresentação de $\overline{P B_{n}(M)}$.

O raciocínio acima é usado para calcular ainda as relações $(\operatorname{Pr} 3),(\operatorname{Pr} 4)$ e $(\operatorname{Pr} 5)$ quando $i \geq 2,(\operatorname{Pr} 6)$ quando $i, j \geq 2$ e $(\operatorname{Pr} 7)$ quando $j \geq 2$ com $U$ sendo a palavra trivial para todas estas relações.

Agora, para finalizar consideremos o relator $(\operatorname{Pr} 8)$ de $P B_{n-1}(M)$ :

$$
\left(\prod_{i=1}^{j-1} T_{j-i, j}^{-1} T_{j-i, j-1}\right)^{-1}\left(a_{j, 1}^{2} \ldots a_{j, g}^{2}\right)^{-1} T_{j, n-1} .
$$

Fazendo uma mudança de índice em $j$ no relator acima obtemos:

$$
\left(\prod_{i=1}^{j-2} T_{(j-1)-i, j-1}^{-1} T_{(j-1)-i, j-2}\right)^{-1}\left(a_{j-1,1}^{2} \ldots a_{j-1, g}^{2}\right)^{-1} T_{j-1, n-1} .
$$

Agora, fazendo uma mudança no índice $i$, obtemos:

$$
\left(\prod_{i=2}^{j-1} T_{(j-1)-(i-1), j-1}^{-1} T_{(j-1)-(i-1), j-2}\right)^{-1}\left(a_{j-1,1}^{2} \ldots a_{j-1, g}^{2}\right)^{-1} T_{j-1, n-1} .
$$

que podemos escrever via homomorfismo $\nu$ :

$$
\nu\left(\left(\prod_{i=2}^{j-1} T_{j-i+1, j}^{-1} T_{j-i+1, j-1}\right)^{-1}\right) \nu\left(\left(a_{j, 1}^{2} \ldots a_{j, g}^{2}\right)^{-1}\right) \nu\left(T_{j, n}\right) .
$$


Assim, pela pré-imagem de $\nu$, temos $\tilde{r} \in P B_{n}(M)$ :

$$
\tilde{r}=\left(\prod_{i=2}^{j-1} T_{j-i+1, j}^{-1} T_{j-i+1, j-1}\right)^{-1}\left(a_{j, 1}^{2} \ldots a_{j, g}^{2}\right)^{-1} T_{j, n}
$$

E fazendo outra mudança de índices para $i$, temos:

$$
\tilde{r}=\left(\prod_{i=1}^{j-2} T_{j-i, j}^{-1} T_{j-i, j-1}\right)^{-1}\left(a_{j, 1}^{2} \ldots a_{j, g}^{2}\right)^{-1} T_{j, n} .
$$

Por outro lado, pela relação $(\operatorname{Pr} 8)$ de $\overline{P B_{n}(M)}$, temos:

$$
\left(\prod_{i=1}^{j-2} T_{j-i, j}^{-1} T_{j-i, j-1}\right)^{-1}\left(a_{j, 1}^{2} \ldots a_{j, g}^{2}\right)^{-1} T_{j, n}=T_{1, j}^{-1} T_{1, j-1} .
$$

Neste caso, temos $U=T_{1, j}^{-1} T_{1, j-1}$. Novamente, $\tilde{r}$ pode ser visto como uma palavra em $G$. Logo, $\tilde{r}=\tilde{\varphi}(U)$, com $\tilde{\varphi}(U)=T_{1, j}^{-1} T_{1, j-1}$ uma palavra em $G_{A}$. Portanto,

$$
\left(\prod_{i=1}^{j-2} T_{j-i, j}^{-1} T_{j-i, j-1}\right)^{-1}\left(a_{j, 1}^{2} \ldots a_{j, g}^{2}\right)^{-1} T_{j, n}=T_{1, j}^{-1} T_{1, j-1}
$$

é a relação de Tipo 2 que queríamos. E ainda é válida em $\overline{P B_{n}(M)}$ pelo mesmo argumento das relações anteriores.

- Finalmente, vamos encontrar as relações de Tipo 3.

Para encontrar tais relações, vamos considerar a apresentação 4 de $P B_{n-1}(M)$. Para cada gerador $x$ de $G_{A}$ e para cada gerador $y$ de $P B_{n-1}(M)$, lembrando que $\tilde{y}$ é a pré-imagem de $y$ por $\nu$, vamos ter as seguintes possibilidades para $\tilde{y} x \tilde{y}^{-1}$ :

$$
A_{i, r} a_{1, r} A_{i, r}^{-1}, A_{i, r} T_{1, j} A_{i, r}^{-1}, T_{j, k} a_{1, r} T_{j, k}^{-1} \text { e } T_{j, k} T_{1, j} T_{j, k}^{-1}
$$

onde $x$ é tal que $x=u(x)$.

Para $i=1$, temos pela relação $(\operatorname{Pr} 2)$ de $P B_{n-1}(M)$ que $A_{j, s} a_{1, r} A_{j, s}^{-1}=a_{1, r}$, com $r \neq s$. Temos também que $a_{1, r}$ é uma palavra em $G$ e $\tilde{\varphi}(V)=a_{1, r}$ é uma palavra em $G_{A}$. Assim, $V=a_{1, r}$. Mais ainda, $A_{j, s} a_{1, r} A_{j, s}^{-1}=a_{1, r}$ é válida em $\overline{P B_{n}(M)}$. Portanto, esta é a primeira relação de Tipo 3 que procurávamos. 
Agora, seja a relação $(\operatorname{Pr} 3)$ de $P B_{n-1}(M) \operatorname{com} i=1$ :

$$
\begin{aligned}
T_{1, j} T_{1, j-1}^{-1} & =\left(a_{1,1}^{2} \ldots a_{1, r-1}^{2} a_{1, r}\right) A_{j, r}\left(a_{1, r}^{-1} a_{1, r-1}^{-2} \ldots a_{1,1}^{-2}\right) A_{j, r}^{-1} \\
& =\left(a_{1,1}^{2} \ldots a_{1, r-1}^{2} a_{1, r}\right) A_{j, r} a_{1, r}^{-1}\left(a_{1, r-1}^{-2} \ldots a_{1,1}^{-2}\right) A_{j, r}^{-1}
\end{aligned}
$$

Logo,

$$
\left(a_{1, r}^{-1} a_{1, r-1}^{-2} \ldots a_{1,1}^{-2}\right) T_{1, j} T_{1, j-1}^{-1}=A_{j, r} a_{1, r}^{-1}\left(a_{1, r-1}^{-2} \ldots a_{1,1}^{-2}\right) A_{j, r}^{-1} .
$$

Usando ( $\operatorname{Pr} 2)$ na equação acima, temos:

$$
A_{j, r} a_{1, r}^{-1} A_{j, r}^{-1}\left(a_{1, r-1}^{-2} \ldots a_{1,1}^{-2}\right)=\left(a_{1, r}^{-1} a_{1, r-1}^{-2} \ldots a_{1,1}^{-2}\right) T_{1, j} T_{1, j-1}^{-1} .
$$

Desta forma, chegamos a seguinte equação:

$$
A_{j, r} a_{1, r} A_{j, r}^{-1}=\left(a_{1, r-1}^{-2} \ldots a_{1,1}^{-2}\right) T_{1, j-1} T_{1, j}^{-1}\left(a_{1,1}^{2} \ldots a_{1, r-1}^{2} a_{1, r}\right) .
$$

Vamos observar que o segundo membro dessa equação é uma palavra em $G$ e, aplicando o homomorfismo $\tilde{\varphi}$ nessa palavra obtemos ela mesma, que é uma palavra em $G_{A}$. Portanto, $\left(a_{1, r-1}^{-2} \ldots a_{1,1}^{-2}\right) T_{1, j-1} T_{1, j}^{-1}\left(a_{1,1}^{2} \ldots a_{1, r-1}^{2} a_{1, r}\right)$ é a palavra $V$ que procuramos e, portanto, $A_{j, r} a_{1, r} A_{j, r}^{-1}=\left(a_{1, r-1}^{-2} \ldots a_{1,1}^{-2}\right) T_{1, j-1} T_{1, j}^{-1}\left(a_{1,1}^{2} \ldots a_{1, r-1}^{2} a_{1, r}\right)$ é uma relação de Tipo 3 que é válida em $\overline{P B_{n}(M)}$ desde que a relação encontrada é uma particularidade da relação $(\operatorname{Pr} 3)$ de $\overline{P B_{n}(M)}$.

De modo totalmente análogo, as relações de Tipo 3 da forma $T_{k, l} T_{1, j} T_{k, l}^{-1}=V$, com $V$ sendo uma palavra em $G_{A}$ seguem das relações $(\operatorname{Pr} 4)$ e $(\operatorname{Pr} 5)$ de $\operatorname{P} B_{n-1}(M)$ enquanto as relações da forma $T_{k, l} a_{1, r} T_{k, l}^{-1}=V$ seguem de $(\operatorname{Pr} 6)$ quando $i=1$. Também, se $j>k$ obtemos de $(\operatorname{Pr} 6)$ as relações de Tipo 3 da forma $A_{j, r} T_{1, k} A_{j, r}^{-1}=V$ : de fato, notemos que $T_{1, k}$ é uma palavra em $G_{A}$. Tomemos $V=T_{1, k}$. Logo, $V=T_{1, k}=A_{j, r} A_{j, r}^{-1} T_{1, k}$. E desde que $j>k$, por $(\operatorname{Pr} 6), A_{j, r}^{-1}$ comuta com $V$. Assim, temos

$$
A_{j, r} T_{1, k} A_{j, r}^{-1}=V
$$

que é ainda válida em $\overline{P B_{n}(M)}$.

Agora, as relações que faltam são aquelas da forma $A_{j, r} T_{1, k} A_{j, r}^{-1}=V$ quando $1<$ $j \leq k$, as quais são deduzidas como segue: por $(\operatorname{Pr} 7)$, sabemos que $a_{j, s}$ comuta com o elemento $a_{1, g}^{-2} \ldots a_{1,1}^{-2} T_{1, k}, s=1, \ldots, g$. Logo, pela definição, temos que $A_{j, s}$ comuta 
com o mesmo elemento.Assim,

$$
\begin{aligned}
\left(a_{1, g}^{-2} \ldots a_{1,1}^{-2} T_{1, k}\right) & =A_{j, r}\left(a_{1, g}^{-2} \ldots a_{1,1}^{-2} T_{1, k}\right) A_{j, r}^{-1} \\
& =\left(A_{j, r} a_{1, g}^{-2} A_{j, r}^{-1}\right) \ldots\left(A_{j, r} a_{1,1}^{-2} A_{j, r}^{-1}\right)\left(A_{j, r} T_{1, k} A_{j, r}^{-1}\right) .
\end{aligned}
$$

Mas usando (Pr2) e (Pr3) sabemos como escrever todos os termos no produto acima (com excessão do termo final da direita) como palavras em $G_{A}$. Assim, isolando o termo final e definindo $V$ como os outros termos que estão no outro membro da equação teremos a equação de Tipo 3 que procurávamos.

E assim, encontramos todas as relações de Tipos 1,2 e 3 de $P B_{n}(M)$. Como todas as relações são válidas em $\overline{P B_{n}(M)}$, provamos ainda que $\tilde{\varphi}$ é injetora. Desta forma, temos o seguinte resultado:

Teorema 5.5.2. Se $M$ for uma superfície fechada, não orientável de gênero $g \geq 2$ então $P B_{n}(M)$ admitirá as Apresentações 3 e 4 definidas anteriormente como apresentações.

Passo 3. Agora, nós queremos encontrar uma apresentação para $B_{n}(M)$, quando $M$ for uma superfície de gênero $g \geq 2$. Vamos definir o grupo $\overline{B_{n}(M)}$ dado pela apresentação do Teorema 5.2.3. Esta é a menor apresentação que encontramos. Mas, para mostrar sua validade, vamos precisar modificá-la um pouco, obtendo uma nova apresentação com mais geradores e mais relações, mas equivalente a primeira.

Primeiro, mudaremos um pouco nossa notação e chamaremos de $a_{1, r}$ os geradores $a_{r}$, para $r=1, \ldots, g$. Então devemos simplesmente adicionar para a apresentação dada os geradores:

- $a_{i, r}$, para $i=2, \ldots, n$ e $r=1, \ldots, g$,

- $T_{j, k}$, para $1 \leq j<k \leq n$.

e vamos incluir também as relações:

(r7) $a_{j+1, r}=\sigma_{j}^{-1} a_{j, r} \sigma_{j}$, para $1 \leq j \leq n-1 ; 1 \leq r \leq g$.

(r8) $T_{j, k}=\sigma_{j} \sigma_{j+1} \ldots \sigma_{k-2} \sigma_{k-1}^{2} \sigma_{k-2} \ldots \sigma_{j}$, para $1 \leq j<k \leq n$.

Claramente, ambas apresentações definem o mesmo grupo, isto é, $\overline{B_{n}(M)}$. Agora, 
vamos definir da forma mais natural possível:

$$
\begin{aligned}
\tilde{\psi}: \overline{B_{n}(M)} \rightarrow B_{n}(M) \\
\sigma_{i} \longmapsto \sigma_{i}, 1 \leq i \leq n-1 \\
\quad a_{i, r} \longmapsto a_{i, r}, 1 \leq i \leq n, 1 \leq r \leq g
\end{aligned}
$$

Usando os mesmos métodos da seção anterior, mostraremos que as relações $(r 7)$ e $(r 8)$ são válidas em $B_{n}(M)$ : de fato, para $(r 7)$, temos:

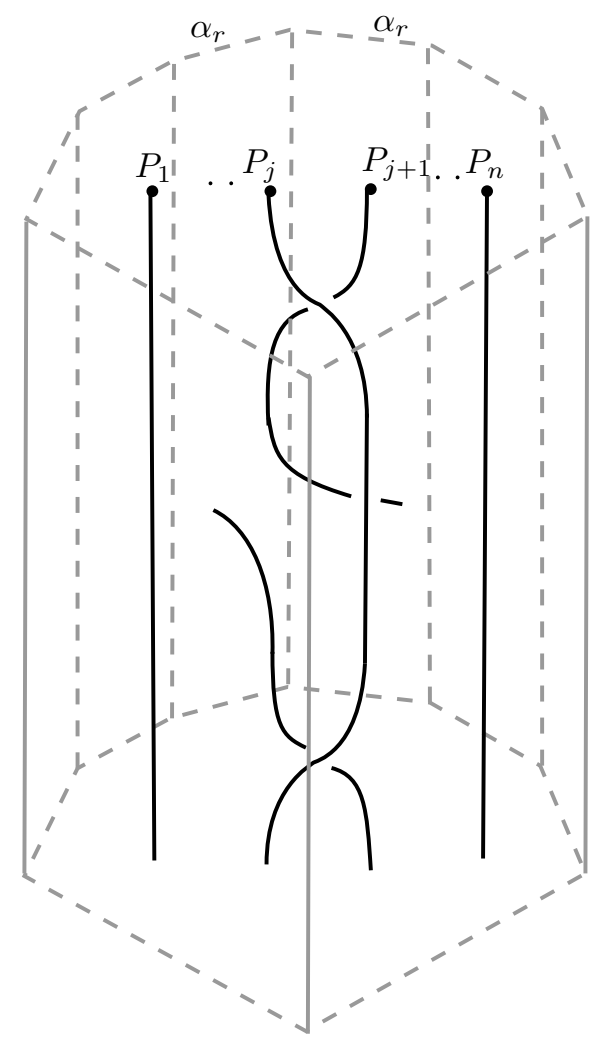

Portanto, a relação $(r 7)$ é válida em $B_{n}(M)$. A relação $(r 8)$ é a mesma trança do caso orientável. Também, mostramos que $(r 1)-(r 6)$ são válidas em $B_{n}(M)$ na primeira seção deste capítulo. Desta forma, temos que $\tilde{\psi}$ é um homomorfismo bem definido.

Agora, consideremos a sequência exata (1):

$$
1 \longrightarrow P B_{n}(M) \stackrel{e}{\longrightarrow} B_{n}(M) \stackrel{f}{\longrightarrow} \Sigma_{n} \longrightarrow 1
$$

Pelo Teorema 5.5.2, temos duas apresentações para $P B_{n}(M)$ (e por ora, vamos considerar a apresentação 3) e para o grupo das permutações $\Sigma_{n}$ temos a mesma apresentação 
que usamos no caso orientável:

\section{Apresentação de $\Sigma_{n}$ :}

Geradores: $\delta_{1}, \ldots, \delta_{n-1}$.

\section{Relações:}

$$
\begin{array}{ll}
-\delta_{i} \delta_{j}=\delta_{j} \delta_{i}, & |i-j| \geq 2, \\
-\delta_{i} \delta_{i+1} \delta_{i}=\delta_{i+1} \delta_{i} \delta_{i+1}, & 1 \leq i \leq n-2, \\
-\delta_{i}^{2}=1, & 1 \leq i \leq n-1,
\end{array}
$$

onde $\delta_{i}$ denota a permutação $(i, i+1)$, para qualquer $i$.

Observemos que $\sigma_{i}$ é uma pré-imagem de $\delta_{i}$ por $f$. Logo, aplicando o Teorema 5.3.1, temos o seguinte conjunto de geradores para $B_{n}(M)$ :

$$
\left\{a_{i, r} ; 1 \leq i \leq n, 1 \leq r \leq g\right\} \cup\left\{T_{j, k} ; 1 \leq j<k \leq n\right\} \cup\left\{\sigma_{i} ; i=1, \ldots, n-1\right\} .
$$

Como $T_{j, k}$ pode ser escrito como combinação dos elementos de $\left\{\sigma_{i} ; i=1, \ldots, n-1\right\}$, podemos simplificar o conjunto de geradores de $B_{n}(M)$ :

$$
\left\{a_{i, r} ; 1 \leq i \leq n, 1 \leq r \leq g\right\} \cup\left\{\sigma_{i} ; i=1, \ldots, n-1\right\},
$$

que é a imagem por $\tilde{\psi}$ dos geradores de $\overline{B_{n}(M)}$ e assim, $\tilde{\psi}$ é sobrejetor.

Analogamente ao que fizemos no passo 2, vamos mostrar que $\tilde{\psi}$ é um isomorfismo pelo procedimento a seguir:

Primeiro, vamos denotar por $G_{A}$ o conjunto de geradores de $P B_{n}(M)$, e por $G$ o conjunto de geradores de $\overline{B_{n}(M)}$. Para cada relação na apresentação de $P B_{n}(M)$, vamos considerá-las via e como relações em $B_{n}(M)$ e mostraremos que são válidas em $\overline{B_{n}(M)}$.

Depois, para cada relator $r$ de $\Sigma_{n}$, vamos considerar sua pré-imagem canônica por $f$, denotada por $\tilde{r}$. Então, encontramos uma palavra $U$ em $G$ tal que $\tilde{r}=U$ vale em $\overline{B_{n}(M)}$, e tal que $\tilde{\psi}(U)$ é uma palavra em $G_{A}$.

Finalmente, para cada $x \in G_{A}$ e cada gerador $\delta_{i}$ de $\Sigma_{n}$, encontramos uma palavra $V$ em $G$ tal que a igualdade $\sigma_{i} x \sigma_{i}^{-1}=V$ vale em $\overline{B_{n}(M)}$ e tal que $\tilde{\psi}(V)$ é uma palavra em $G_{A}$. 
Isto nos dá as relacões de Tipo 1, 2 e 3 do Teorema 5.3.1 e, portanto, uma apresentação de $B_{n}(M)$, e, ao mesmo tempo mostramos que $\tilde{\psi}$ é injetora, e, consequentemente, que $\tilde{\psi}$ é um isomorfismo.

- Vamos então verificar em $\overline{B_{n}(M)}$ as relações de Tipo 1. Partiremos de (r3) para chegar na relação $(\operatorname{Pr} 1)$ :

$$
a_{1,1}^{2} \ldots a_{1, g}^{2}=\sigma_{1} \ldots \sigma_{n-2} \sigma_{n-1}^{2} \sigma_{n-2} \ldots \sigma_{1}
$$

Substituindo cada $a_{i, r}, i=1, \ldots, n-1$ por $(r 7)$, temos:

$$
\begin{aligned}
\sigma_{1} \ldots \sigma_{n-2} \sigma_{n-1}^{2} \sigma_{n-2} \ldots \sigma_{1} & =a_{1,1}^{2} \ldots a_{1, g}^{2} \\
& =\left(\sigma_{1} a_{2,1}^{2} \sigma_{1}^{-1}\right) \ldots\left(\sigma_{1} a_{2, g}^{2} \sigma_{1}^{-1}\right) \\
& =\sigma_{1}\left(a_{2,1}^{2} \ldots a_{2, g}^{2}\right) \sigma_{1}^{-1} \\
& =\cdots \\
& =\sigma_{1} \sigma_{2} \ldots \sigma_{n-1}\left(a_{n, 1}^{2} \ldots a_{n, g}^{2}\right) \sigma_{n-1}^{-1} \ldots \sigma_{2}^{-1} \sigma_{1}^{-1}
\end{aligned}
$$

Desta forma a equação $(*)$ fica:

$$
a_{n, 1}^{2} \ldots a_{n, g}^{2}=\sigma_{n-1} \ldots \sigma_{1}^{2} \ldots \sigma_{n-1}
$$

Mas já provamos que $\prod_{i=1}^{n-1} T_{i, n-1}^{-1} T_{i, n}=\sigma_{n-1} \ldots \sigma_{1}^{2} \ldots \sigma_{n-1}$. Portanto:

$$
a_{n, 1}^{2} \ldots a_{n, g}^{2}=\prod_{i=1}^{n-1} T_{i, n-1}^{-1} T_{i, n}
$$

E assim, temos a relação $(\operatorname{Pr} 1)$. Via homomorfismo e, $(\operatorname{Pr} 1)$ é uma relação de $B_{n}(M)$ de Tipo 1. Mais ainda, esta relação é válida em $\overline{B_{n}(M)}$.

Usaremos daqui para frente algumas relações de $\overline{B_{n}(M)}$ facilmente deduzidas de 
$(r 1)-(r 8)$. Para a primeira relação, usando $(r 7)$, temos:

$$
\begin{aligned}
\left(\sigma_{i-1}^{-1} \ldots \sigma_{1}^{-1}\right) a_{1, r}\left(\sigma_{1} \ldots \sigma_{i-1}\right) & =\sigma_{i-1}^{-1} \ldots \sigma_{2}^{-1}\left(\sigma_{1}^{-1} a_{1, r} \sigma_{1}\right) \sigma_{2} \ldots \sigma_{i-1} \\
& =\cdots \\
& =\sigma_{i-1}^{-1} a_{i-1, r} \sigma_{i-1} \\
& =a_{i, r}
\end{aligned}
$$

Portanto,

$$
a_{i, r}=\left(\sigma_{i-1}^{-1} \ldots \sigma_{1}^{-1}\right) a_{1, r}\left(\sigma_{1} \ldots \sigma_{i-1}\right)
$$

Outras duas relações são obtidas de $(r 7)$ :

$$
\begin{aligned}
\left(\sigma_{j-1}^{-1} \ldots \sigma_{2}^{-1}\right) A_{2, s}\left(\sigma_{2} \ldots \sigma_{j-1}\right) & =\left(\sigma_{j-1}^{-1} \ldots \sigma_{2}^{-1}\right)\left(a_{2,1}^{2} \ldots a_{2, s-1}^{2} a_{2, s}^{-1} a_{2, s-1}^{-2} \ldots a_{2,1}^{-1}\right)\left(\sigma_{2} \ldots \sigma_{j-1}\right) \\
& =\sigma_{j-1}^{-1} \ldots \sigma_{3}^{-1}\left(a_{3,1}^{2} \ldots a_{3, s-1}^{2} a_{3, s}^{-1} a_{3, s-1}^{-2} \ldots a_{3,1}^{-2}\right)\left(\sigma_{3} \ldots \sigma_{j-1}\right) \\
& =\cdots \\
& =\sigma_{j-1}^{-1} a_{j-1, s} \sigma_{j-1} \\
& =A_{j, s} .
\end{aligned}
$$

E com o raciocínio análogo, mostramos que:

$$
\left(\sigma_{j-1}^{-1} \ldots \sigma_{1}^{-1}\right) A_{1, s}\left(\sigma_{1} \ldots \sigma_{j-1}\right)=A_{j, s}
$$

Portanto,

$$
A_{j, s}=\left(\sigma_{j-1}^{-1} \ldots \sigma_{1}^{-1}\right) A_{1, s}\left(\sigma_{1} \ldots \sigma_{j-1}\right)=\left(\sigma_{j-1}^{-1} \ldots \sigma_{2}^{-1}\right) A_{2, s}\left(\sigma_{2} \ldots \sigma_{j-1}\right)
$$

Da mesma forma que no caso orientável, teremos as três relações que extraímos das relações $(r 1)$ e $(r 2)$ do disco:

$$
\begin{gathered}
\sigma_{j}\left(\sigma_{k} \sigma_{k-1} \ldots \sigma_{i}\right)=\left(\sigma_{k} \sigma_{k-1} \ldots \sigma_{i}\right) \sigma_{j+1}, i \leq j<k . \\
\sigma_{j}\left(\sigma_{k}^{-1} \sigma_{k-1}^{-1} \ldots \sigma_{i}^{-1}\right)=\left(\sigma_{k}^{-1} \sigma_{k-1}^{-1} \ldots \sigma_{i}^{-1}\right) \sigma_{j+1}, i \leq j<k . \\
\sigma_{i} \ldots \sigma_{k-1} \sigma_{k}^{2} \sigma_{k-1} \ldots \sigma_{i}^{-1}=\sigma_{k}^{-1} \ldots \sigma_{i+1}^{-1} \sigma_{i}^{2} \sigma_{i+1} \ldots \sigma_{k} .
\end{gathered}
$$


Agora, usando $\left(5^{\prime}\right),\left(6^{\prime}\right),\left(7^{\prime}\right)$ e $(R 6)$, vemos que se $1 \leq k \leq j-2$, temos:

$$
\begin{aligned}
\sigma_{k} A_{j, s} & =\sigma_{k}\left(\sigma_{j-1}^{-1} \ldots \sigma_{1}^{-1}\right) A_{1, s}\left(\sigma_{1} \ldots \sigma_{j-1}\right) \\
& =\left(\sigma_{j-1}^{-1} \ldots \sigma_{1}^{-1}\right) \sigma_{k+1} A_{1, s}\left(\sigma_{1} \ldots \sigma_{j-1}\right) \\
& =\left(\sigma_{j-1}^{-1} \ldots \sigma_{1}^{-1}\right) A_{1, s} \sigma_{k+1}\left(\sigma_{1} \ldots \sigma_{j-1}\right) \\
& =\left(\sigma_{j-1}^{-1} \ldots \sigma_{1}^{-1}\right) A_{1, s}\left(\sigma_{1} \ldots \sigma_{j-1}\right) \sigma_{k+1} \\
& =A_{j, s} \sigma_{k} .
\end{aligned}
$$

Ou seja,

$$
\sigma_{k} A_{j, s}=A_{j, s} \sigma_{k}
$$

Da mesma forma, usando $\left(5^{\prime}\right),(r 4)$ e $(r 6)$, temos para $r \neq s, 1<j$ :

$$
\begin{aligned}
a_{1, r} A_{j, s} & =a_{1, r}\left(\sigma_{j-1}^{-1} \ldots \sigma_{2}^{-1}\right) A_{2, s}\left(\sigma_{j-1} \ldots \sigma_{2}\right) \\
& =\left(\sigma_{j-1}^{-1} \ldots \sigma_{2}^{-1}\right) a_{1, r} A_{2, s}\left(\sigma_{j-1} \ldots \sigma_{2}\right) \\
& =\left(\sigma_{j-1}^{-1} \ldots \sigma_{2}^{-1}\right) A_{2, s} a_{1, r}\left(\sigma_{j-1} \ldots \sigma_{2}\right) \\
& =\left(\sigma_{j-1}^{-1} \ldots \sigma_{2}^{-1}\right) A_{2, s}\left(\sigma_{j-1} \ldots \sigma_{2}\right) a_{1, r} \\
& =A_{j, s} a_{1, r} .
\end{aligned}
$$

Portanto, $a_{1, r} A_{j, s}=A_{j, s} a_{1, r}, r \neq s, 1<j$. E como em $\left(4^{\prime}\right) a_{i, r}$ é um produto de elementos o qual comuta com $A_{j, s}$, obtemos:

$$
a_{i, r} A_{j, s}=A_{j, s} a_{i, r}, i<j, r \neq s
$$

ou seja, $(\operatorname{Pr} 2)$ é válida em $\overline{B_{n}(M)}$. Mais ainda, $(\operatorname{Pr} 2)$ é uma relação de Tipo 1 via o homomorfismo e para $B_{n}(M)$.

Agora, vamos verificar a relação (Pr3). Em cada uma das igualdades a seguir serão usadas as relações conhecidas de $\overline{B_{n}(M)}$ :

$$
\begin{aligned}
& T_{i, j} T_{i, j-1}^{-1} A_{j, r}\left(a_{i, 1}^{2} \ldots a_{i, r-1}^{2} a_{i, r}\right) \\
& =\left(\sigma_{i} \ldots \sigma_{j-2} \sigma_{j-1}^{2} \sigma_{j-2}^{-1} \ldots \sigma_{i}^{-1}\right) A_{j, r}\left(a_{i, 1}^{2} \ldots a_{i, r-1}^{2} a_{i, r}\right) \\
& =\left(\sigma_{i} \ldots \sigma_{j-2} \sigma_{j-1}^{2} \sigma_{j-2}^{-1} \ldots \sigma_{i}^{-1}\right) A_{j, r}\left(\sigma_{i-1}^{-1} \ldots \sigma_{1}^{-1}\right)\left(a_{1,1}^{2} \ldots a_{1, r-1}^{2} a_{1, r}\right)\left(\sigma_{1} \ldots \sigma_{i-1}\right) \\
& =\left(\sigma_{i} \ldots \sigma_{j-2} \sigma_{j-1}^{2} \sigma_{j-2}^{-1} \ldots \sigma_{i}^{-1}\right)\left(\sigma_{i-1}^{-1} \ldots \sigma_{1}^{-1}\right) A_{j, r}\left(a_{1,1}^{2} \ldots a_{1, r-1}^{2} a_{1, r}\right)\left(\sigma_{1} \ldots \sigma_{i-1}\right) \\
& =\left(\sigma_{i} \ldots \sigma_{j-2} \sigma_{j-1}^{2} \sigma_{j-2}^{-1} \ldots \sigma_{1}^{-1}\right) A_{j, r}\left(a_{1,1}^{2} \ldots a_{1, r-1}^{2} a_{1, r}\right)\left(\sigma_{1} \ldots \sigma_{i-1}\right)
\end{aligned}
$$




$$
\begin{aligned}
& =\left(\sigma_{i} \ldots \sigma_{j-2} \sigma_{j-1}^{2} \sigma_{j-2}^{-1} \ldots \sigma_{1}^{-1}\right)\left(\sigma_{j-1}^{-1} \ldots \sigma_{2}^{-1}\right) A_{2, r}\left(\sigma_{2} \ldots \sigma_{j-1}\right)\left(a_{1,1}^{2} \ldots a_{1, r-1}^{2} a_{1, r}\right)\left(\sigma_{1} \ldots \sigma_{i-1}\right) \\
& =\left(\sigma_{i-1}^{-1} \ldots \sigma_{1}^{-1}\right)\left(\sigma_{1} \ldots \sigma_{i-1}\right)\left(\sigma_{i} \ldots \sigma_{j-2} \sigma_{j-1}^{2} \sigma_{j-2}^{-1} \ldots \sigma_{1}^{-1}\right)\left(\sigma_{j-1}^{-1} \ldots \sigma_{2}^{-1}\right) A_{2, r}\left(a_{1,1}^{2} \ldots a_{1, r-1}^{2} a_{1, r}\right) \\
& \left(\sigma_{2} \ldots \sigma_{j-1}\right)\left(\sigma_{1} \ldots \sigma_{i-1}\right) \\
& =\left(\sigma_{i-1}^{-1} \ldots \sigma_{1}^{-1}\right)\left(\sigma_{j-1}^{-1} \ldots \sigma_{2}^{-1} \sigma_{1}^{2}\right) A_{2, r}\left(a_{1,1}^{2} \ldots a_{1, r-1}^{2} a_{1, r}\right)\left(\sigma_{2} \ldots \sigma_{j-1}\right)\left(\sigma_{1} \ldots \sigma_{i-1}\right) \\
& =\left(\sigma_{i-1}^{-1} \ldots \sigma_{1}^{-1}\right)\left(\sigma_{j-1}^{-1} \ldots \sigma_{2}^{-1}\right)\left(a_{1,1}^{2} \ldots a_{1, r-1}^{2} a_{1, r}\right) A_{2, r}\left(\sigma_{2} \ldots \sigma_{j-1}\right)\left(\sigma_{1} \ldots \sigma_{i-1}\right) \\
& =\left(\sigma_{i-1}^{-1} \ldots \sigma_{1}^{-1}\right)\left(a_{1,1}^{2} \ldots a_{1, r-1}^{2} a_{1, r}\right)\left(\sigma_{j-1}^{-1} \ldots \sigma_{2}^{-1}\right) A_{2, r}\left(\sigma_{2} \ldots \sigma_{j-1}\right)\left(\sigma_{1} \ldots \sigma_{i-1}\right) \\
& =\left(\sigma_{i-1}^{-1} \ldots \sigma_{1}^{-1}\right)\left(a_{1,1}^{2} \ldots a_{1, r-1}^{2} a_{1, r}\right) A_{j, r}\left(\sigma_{1} \ldots \sigma_{i-1}\right) \\
& =\left(\sigma_{i-1}^{-1} \ldots \sigma_{1}^{-1}\right)\left(a_{1,1}^{2} \ldots a_{1, r-1}^{2} a_{1, r}\right)\left(\sigma_{1} \ldots \sigma_{i-1}\right) A_{j, r} \\
& =\left(a_{i, 1}^{2} \ldots a_{i, r-1}^{2} a_{i, r}\right) A_{j, r} .
\end{aligned}
$$

E portanto, temos $(\operatorname{Pr} 3)$. As relações $(\operatorname{Pr} 4)$ e $(\operatorname{Pr} 5)$ são tranças que não atravessam paredes, e assim são relações no grupo de tranças no disco, logo são consequências de $(r 1)$ e $(r 2)$.

Agora, vamos mostrar que $(\operatorname{Pr} 6)$ é válido em $\overline{B_{n}(M)}$ para $1 \leq j<k<i \leq n$ :

$$
\begin{aligned}
a_{i, r} T_{j, k} & =\left(\sigma_{i-1}^{-1} \ldots \sigma_{1}^{-1}\right) a_{1, r}\left(\sigma_{1} \ldots \sigma_{i-1}\right) T_{j, k} \\
& =\left(\sigma_{i-1}^{-1} \ldots \sigma_{1}^{-1}\right) a_{1, r}\left(\sigma_{1} \ldots \sigma_{i-1}\right)\left(\sigma_{j} \sigma_{j+1} \ldots \sigma_{k-2} \sigma_{k-1}^{2} \sigma_{k-2} \ldots \sigma_{j+1} \sigma_{j}\right) \\
& =\left(\sigma_{i-1}^{-1} \ldots \sigma_{1}^{-1}\right) a_{1, r}\left(\sigma_{j} \sigma_{j+1} \ldots \sigma_{k-2} \sigma_{k-1}^{2} \sigma_{k-2} \ldots \sigma_{j+1} \sigma_{j}\right)\left(\sigma_{1} \ldots \sigma_{i-1}\right) \\
& =\left(\sigma_{i-1}^{-1} \ldots \sigma_{1}^{-1}\right)\left(\sigma_{j} \sigma_{j+1} \ldots \sigma_{k-2} \sigma_{k-1}^{2} \sigma_{k-2} \ldots \sigma_{j+1} \sigma_{j}\right) a_{1, r}\left(\sigma_{1} \ldots \sigma_{i-1}\right) \\
& =\left(\sigma_{j} \sigma_{j+1} \ldots \sigma_{k-2} \sigma_{k-1}^{2} \sigma_{k-2} \ldots \sigma_{j+1} \sigma_{j}\right)\left(\sigma_{i-1}^{-1} \ldots \sigma_{1}^{-1}\right) a_{1, r}\left(\sigma_{1} \ldots \sigma_{i-1}\right) \\
& =T_{j, k} a_{i, r} .
\end{aligned}
$$

E assim, o resultado segue.

Agora, a relação $(\operatorname{Pr} 7)$ é obtida a seguir:

$$
\begin{aligned}
a_{1, r}\left(a_{j, g}^{-2} \ldots a_{j, 1}^{-2} T_{j, k}\right) & =a_{i, r}\left(\sigma_{j-1}^{-1} \ldots \sigma_{1}^{-1} a_{1, g}^{-2} \ldots a_{1,1}^{-2} \sigma_{1} \ldots \sigma_{k-1}\right) \sigma_{k-1} \ldots \sigma_{j} \\
& =a_{i, r}\left(\sigma_{j-1}^{-1} \ldots \sigma_{1}^{-1}\right)\left(\sigma_{1}^{-1} \ldots \sigma_{n-1}^{-1}\right)\left(\sigma_{n-1}^{-1} \ldots \sigma_{k}^{-1}\right)\left(\sigma_{k-1} \ldots \sigma_{j}\right) \\
& =\left(\sigma_{j-1}^{-1} \ldots \sigma_{1}^{-1}\right)\left(\sigma_{1}^{-1} \ldots \sigma_{i-2}^{-1}\right) a_{i, r} \sigma_{i-1}^{-1}\left(\sigma_{i}^{-1} \ldots \sigma_{n-1}^{-1}\right)\left(\sigma_{n-1}^{-1} \ldots \sigma_{k}^{-1}\right)\left(\sigma_{k-1} \ldots \sigma_{j}\right) \\
& =\left(\sigma_{j-1}^{-1} \ldots \sigma_{1}^{-1}\right)\left(\sigma_{1}^{-1} \ldots \sigma_{i-2}^{-1}\right) \sigma_{i-1}^{-1} a_{i-1, r}\left(\sigma_{i}^{-1} \ldots \sigma_{n-1}^{-1}\right)\left(\sigma_{n-1}^{-1} \ldots \sigma_{k}^{-1}\right)\left(\sigma_{k-1} \ldots \sigma_{j}\right) \\
& =\left(\sigma_{j-1}^{-1} \ldots \sigma_{1}^{-1}\right)\left(\sigma_{1}^{-1} \ldots \sigma_{n-1}^{-1}\right)\left(\sigma_{n-1}^{-1} \ldots \sigma_{k}^{-1}\right)\left(\sigma_{k-1} \ldots \sigma_{j}\right) a_{i-1, r} \sigma_{i-1} \sigma_{i-2} \ldots \sigma_{j} \\
& =\left(\sigma_{j-1}^{-1} \ldots \sigma_{1}^{-1}\right)\left(\sigma_{1}^{-1} \ldots \sigma_{n-1}^{-1}\right)\left(\sigma_{n-1}^{-1} \ldots \sigma_{k}^{-1}\right)\left(\sigma_{k-1} \ldots \sigma_{j}\right) a_{i, r} \\
& =\left(a_{j, g}^{-2} \ldots a_{j, 1}^{-2} T_{j, k}\right) a_{i, r} .
\end{aligned}
$$


Finalmente, a relação $(\operatorname{Pr} 8)$ é verificada da seguinte forma:

$$
\begin{aligned}
a_{j, 1}^{2} \ldots a_{j, g}^{2}\left(\prod_{i=1}^{j-1} T_{j-i, j}^{-1} T_{j-i, j-1}\right) & =a_{j, 1}^{2} \ldots a_{j, g}^{2}\left(\sigma_{j-1}^{-1} \ldots \sigma_{1}^{-2} \ldots \sigma_{j-1}^{-1}\right) \\
& =\sigma_{j-1}^{-1} \ldots \sigma_{1}^{-1} a_{1,1}^{2} \ldots a_{1, g}^{2} \sigma_{1}^{-1} \ldots \sigma_{j-1}^{-1} \\
& =\sigma_{j} \ldots \sigma_{n-1}^{2} \ldots \sigma_{j} \\
& =T_{j, n} .
\end{aligned}
$$

E aqui, terminamos as relações de Tipo 1.

- Vamos encontrar agora as relações de Tipo 2. Para cada relator na apresentação de $\Sigma_{n}$, nós devemos encontrar uma palavra $U$ mencionada anteriormente. O primeiro relator em $\Sigma_{n}$ é $\delta_{i} \delta_{j} \delta_{i}^{-1} \delta_{j}^{-1}$, quando $|i-j| \geq 2$. Podemos escrevê-lo como:

$$
f\left(\sigma_{i}\right) f\left(\sigma_{j}\right) f\left(\sigma_{i}^{-1}\right) f\left(\sigma_{j}^{-1}\right) .
$$

Logo, teremos $\tilde{r}=\sigma_{i} \sigma_{j} \sigma_{i}^{-1} \sigma_{j}^{-1}$. Por outro lado, por $(r 1)$ em $\overline{B_{n}(M)}$ :

$$
\sigma_{i} \sigma_{j} \sigma_{i}^{-1} \sigma_{j}^{-1}=1,|i-j| \geq 2
$$

Vamos observar que 1 é uma palavra em $G_{A}$. Portanto, definimos $U=1$. Assim $\tilde{r}=1$ é uma relação de Tipo 2 que procuramos e mais ainda, válida em $\overline{B_{n}(M)}$.

O segundo relator em $\Sigma_{n}$ é $\delta_{i} \delta_{i+1} \delta_{i} \delta_{i+1}^{-1} \delta_{i}^{-1} \delta_{i+1}^{-1}$. Podemos escrevê-lo como

$$
f\left(\sigma_{i}\right) f\left(\sigma_{i+1}\right) f\left(\sigma_{i}\right) f\left(\sigma_{i+1}^{-1}\right) f\left(\sigma_{i}^{-1}\right) f\left(\sigma_{i+1}^{-1}\right), i=1, \ldots, n-2 .
$$

Logo, teremos $\tilde{r}=\sigma_{i} \sigma_{i+1} \sigma_{i} \sigma_{i+1}^{-1} \sigma_{i}^{-1} \sigma_{i+1}^{-1}$. Por outro lado, por $(r 2)$ em $\overline{B_{n}(M)}$ :

$$
\sigma_{i} \sigma_{i+1} \sigma_{i} \sigma_{i+1}^{-1} \sigma_{i}^{-1} \sigma_{i+1}^{-1}=1 ; i=1, \ldots, n-2 \text {. }
$$

Como no caso anterior, $U=1$. Logo, temos a segunda relação de Tipo 2 que procurávamos a qual é válida em $\overline{B_{n}(M)}$.

E para a última relação de Tipo 2, consideremos o relator $\delta_{i}^{2}$ de $\Sigma_{n}$ que podemos 
escrever como $f\left(\sigma_{i}^{2}\right)$. Logo, $\tilde{r}=\sigma_{i}^{2}$. Por outro lado, por $(r 8)$ temos:

$$
\sigma_{i}^{2}=T_{i, i+1}
$$

e observemos que $T_{i, i+1}$ é uma palavra em $G_{A}$. Logo, $U=T_{i, i+1}$ e assim, temos nossa última relação de Tipo 2 que também é válida em $\overline{B_{n}(M)}$.

- Vamos terminar a prova do Teorema 5.2.3 obtendo as relações de Tipo 3 e mostrando que estas são válidas em $\overline{B_{n}(M)}$. Elas serão fáceis de deduzir e, para isto, usaremos as relações $\left(9^{\prime}\right),(r 1),(r 2),(r 7)$ e $(r 8)$. Consideremos a apresentação 3 de $P B_{n}(M)$.

Para cada gerador $x$ de $G_{A}$ e para cada gerador $y$ de $\Sigma_{n}$, vamos determinar tais relações: por $\left(9^{\prime}\right)$, temos $\sigma_{i} A_{j, r} \sigma_{i}^{-1}=A_{j, r}, 1 \leq i \leq j-2$. Então, temos que a trança $\sigma_{i}$ comuta com $a_{j, r}$, para $j \neq i, i+1$, ou seja, $\sigma_{i} a_{j, r} \sigma_{i}^{-1}=a_{j, r}$. Vamos observar que $a_{j, r}$ é uma palavra em $G_{A}$. Logo, tomamos $V=a_{j, r}$ e assim temos a primeira relação de Tipo 3 que procuramos.

Agora, por $(r 7)$ temos $a_{i+1, r}=\sigma_{i}^{-1} a_{i, r} \sigma_{i}, 1 \leq i \leq n-1$. Por $(r 9)$ temos $T_{i, i+1}=\sigma_{i}^{2}$, $\operatorname{logo}, T_{i, i+1}^{-1}=\sigma_{i}^{-2}$. Portanto,

$$
\begin{aligned}
\sigma_{i} a_{i, r} \sigma_{i}^{-1} & =\sigma_{i}^{2} a_{i+1, r} \sigma_{i}^{-2} \\
& =T_{i, i+1} a_{i+1, r} T_{i, i+1}^{-1}
\end{aligned}
$$

ou seja, $\sigma_{i} a_{i, r} \sigma_{i}^{-1}=a_{i+1, r} T_{i, i+1}^{-1}$. Pelo mesmo raciocínio anterior, $V=T_{i, i+1} a_{i+1, r} T_{i, i+1}^{-1}$ e assim encontramos mais uma relação de Tipo 3 .

Também, pela relação $(r 7)$, temos $\sigma_{i} a_{i+1, r} \sigma_{i}^{-1}=a_{i, r}$ e $a_{i, r}$ é uma palavra em $G_{A}$. Portanto, tomando $V=a_{i, r}$ obtemos mais uma relação de Tipo 3 .

As relações de Tipo 3 das formas: $\sigma_{i} T_{j, k} \sigma_{i}^{-1}=T_{j, k}, \operatorname{com} i \neq j-1, j, k, \sigma_{i} T_{i+1, k} \sigma_{i}^{-1}=$ $T_{i, k} T_{i, i+1}^{-1}, \sigma_{i} T_{i, k} \sigma_{i}^{-1}=T_{i, i+1} T_{i+1, k}$ e $\sigma_{i} T_{j, i} \sigma_{i}^{-1}=T_{j, i-1} T_{j, i}^{-1} T_{j, i+1}$ são as mesmas do caso orientável.

E, claramente, todas as relações são válidas em $\overline{B_{n}(M)}$. E aqui, as relações de Tipo 3 chegam ao fim.

Como todas as relações do Tipo 1,2 e 3 são válidas em $\overline{B_{n}(M)}$ então segue que $\tilde{\psi}$ é um isomorfismo e portanto, o Teorema 5.2.3 fica provado. 


\subsection{O problema da palavra}

Nesta seção vamos fazer uma aplicação dos resultados obtidos nas seções 4 e 5 deste mesmo capítulo. Vamos fornecer um algoritmo para resolver o problema da palavra no grupo de tranças em superfícies e, para isto, vamos precisar das apresentações que estudamos nas duas seções anteriores.

Faremos aqui, uma breve discussão para o caso em que $M$ é uma superfície fechada, orientável de gênero $g \geq 1$, mas observemos que o caso em que $M$ é uma superfície fechada, não orientável de gênero $g \geq 2$ é análogo.

Seja $w$ uma palavra nos geradores de $B_{n}(M)$, isto é, uma palavra composta por elementos do conjunto $\left\{\sigma_{1}, \ldots, \sigma_{n-1}, a_{1,1}, \ldots, a_{1,2 g}\right\}$ e por seus inversos. O algoritmo que vamos propor nos dá como saída uma palavra

$$
w^{\prime}=w_{1} \ldots w_{n} s
$$

tal que:

- $w^{\prime}$ é equivalente a $w$;

- cada $w_{i}$, com $i=1, \ldots, n$ é uma palavra composta por elementos do conjunto $\left\{a_{i, 1}, \ldots, a_{i, 2 g}, T_{i, i+1}, \ldots, T_{i, n-1}\right\}$ e $s$ é uma palavra composta por elementos do conjunto $\left\{\sigma_{1}, \ldots, \sigma_{n-1}\right\}$ que representa a permutação que $w$ induz nas suas cordas.

Mostraremos ainda, que esta expressão acima é única. Desta forma, teremos $w=1$ se, e somente se, $w^{\prime}$ for a palavra trivial. Este algoritmo é análogo ao método clássico de pentear tranças no grupo de tranças no disco, vide [BZ] (páginas 149 - 153).

Primeiro, vamos considerar o homomorfismo $f$ na sequência exata (1):

$$
1 \longrightarrow P B_{n}(M) \longrightarrow B_{n}(M) \stackrel{f}{\longrightarrow} \Sigma_{n} \longrightarrow 1
$$

Este homomorfismo leva a palavra $w$ na sua permutação correspondente. Agora, para qualquer elemento de $\Sigma_{n}$, podemos tomar uma forma normal, isto é, uma palavra única composta por elementos do conjunto das permutações $\left\{\delta_{1}, \ldots, \delta_{n-1}\right\}$. Por exemplo, podemos usar a forma normal descrita em $[\mathrm{H}]$, onde qualquer elemento de $\Sigma_{n}$ é escrito como um produto:

$$
t_{1, k_{1}} t_{2, k_{2}} \ldots t_{n-1, k_{n-1}},
$$


onde $t_{m, 0}=1$ e $t_{m, k}=\delta_{m} \delta_{m-1} \ldots \delta_{m-k+1}$. Se substituirmos nesta forma normal o elemento $\delta_{i}$ pelo elemento $\sigma_{i}$ para $i=1, \ldots, n-1$ obteremos uma aplicação $g: \Sigma_{n} \rightarrow W$, onde $W$ é o conjunto de palavras em $\left\{\sigma_{1}, \ldots, \sigma_{n-1}\right\}$ e seus inversos.

Desta forma, vamos considerar a composição $\epsilon=g \circ f$ :

$$
\epsilon: B_{n}(M) \stackrel{f}{\longrightarrow} \Sigma_{n} \stackrel{g}{\longrightarrow} W
$$

Esta aplicação leva qualquer trança para uma "palavra trança" induzindo a mesma permutação nas cordas. Mais ainda, a imagem de $\epsilon$ é finita desde que $\Sigma_{n}$ é finito.

Vamos definir $s=\epsilon(w)$. Desde que as formas normais de $\Sigma_{n}$ são únicas, então $s$ é única. Assim, obtemos uma palavra $\bar{w}=w s^{-1} \in P B_{n}(M)$ tal que $w=\bar{w} s$.

Agora, vamos proceder como segue: primeiro, reescrevemos $\bar{w}$ em termos dos geradores de $P B_{n}(M),\left\{a_{i, r}, T_{j, k}\right\}$, e então aplicaremos o método de pentear tranças de Artin.

Reescrevendo em termos dos geradores de $P B_{n}(M)$ :

Para todas as palavras tranças $p$ na imagem de $\epsilon$, consideremos as seguintes tranças:

$$
p a_{1, r}^{ \pm 1} \epsilon\left(a_{1, r}^{ \pm 1}\right) p^{-1}, \quad p \sigma_{i}^{ \pm 1} \epsilon\left(\sigma_{i}^{ \pm 1}\right) p^{-1}
$$

Claramente, existe apenas um número finito delas e elas são tranças puras. Não é difícil reescrever estas tranças como palavras em $\left\{a_{i, r}, T_{j, k}\right\}$ usando as relações dadas na apresentação de $B_{n}(M)$.

Suponhamos que $\bar{w}$ tenha comprimento $m$, isto é, $\bar{w}=x_{1} \ldots x_{m}$ onde $x_{i}$ é um gerador de $B_{n}(M)$ ou seu inverso. Para todo $i=1, \ldots, m$ vamos definir $\bar{w}_{i}=x_{1} \ldots x_{i}$. Desde que $\bar{w} \in P B_{n}(M)$, então $\epsilon\left(\bar{w}_{m}\right)=\epsilon(\bar{w})=1$ e assim temos:

$$
\bar{w}=x_{1} \ldots x_{m}=\left(1 x_{1} \epsilon\left(\bar{w}_{1}\right)^{-1}\right)\left(\epsilon\left(\bar{w}_{1}\right) x_{2} \epsilon\left(\bar{w}_{2}\right)^{-1}\right) \ldots\left(\epsilon\left(\bar{w}_{m-1}\right) x_{m} \epsilon\left(\bar{w}_{m}\right)^{-1}\right)
$$

Mas já sabemos como reescrever, como palavras em $\left\{a_{i, r} T_{j, k}\right\}$, todos os fatores do lado direito da equação. Então podemos reescrever $\bar{w}$ como uma palavra nos geradores de $P B_{n}(M)$.

\section{Método para pentear tranças:}


Para $j=1, \ldots, n$ definimos os seguintes conjuntos:

$$
\begin{aligned}
& W_{j}=\left\{a_{i, r}^{ \pm 1} ; i=1, \ldots, j, r=1, \ldots, 2 g\right\} \cup\left\{T_{i, k}^{ \pm 1} ; i=1, \ldots, j, k=i+1, \ldots, n-1\right\} \\
& V_{j}=\left\{A_{j, r}^{ \pm 1} ; r=1, \ldots, 2 g\right\} \cup\left\{T_{j, k}^{ \pm 1} ; k=j+1, \ldots, n-1\right\} .
\end{aligned}
$$

Para cada $x \in W_{i}$, e para cada $y \in V_{j}, i<j$, podemos escrever

$$
y x y^{-1}=Z
$$

onde $Z$ é uma palavra em $W_{i}$. Para vermos este fato, tomemos $x=a_{i, r} \in W_{i}$ e $y=T_{j, k} \in$ $V_{j}$. Assim, pela definição dos índices $i, j$ e $k$ em $W_{i}$ e $V_{j}$ e, pela relação de Tipo 3:

$$
\sigma_{j} a_{i, r} \sigma_{j}^{-1}=a_{i, r}
$$

temos o seguinte:

$$
\begin{aligned}
y x y^{-1} & =T_{j, k} a_{i, r} T_{j, k}^{-1} \\
& =\left(\sigma_{j} \ldots \sigma_{k-2} \sigma_{k-1}^{2} \sigma_{k-2} \ldots \sigma_{j}\right) a_{i, r}\left(\sigma_{j}^{-1} \ldots \sigma_{k-2}^{-1} \sigma_{k-1}^{-2} \sigma_{k-2}^{-1} \ldots \sigma_{j}^{-1}\right) \\
& =\left(\sigma_{j} \ldots \sigma_{k-2} \sigma_{k-1}^{2} \sigma_{k-2} \ldots \sigma_{j-1}\right) a_{i, r}\left(\sigma_{j-1}^{-1} \ldots \sigma_{k-2}^{-1} \sigma_{k-1}^{-2} \sigma_{k-2}^{-1} \ldots \sigma_{j-1}^{-1}\right) \\
& =\cdots \\
& =a_{i, r}
\end{aligned}
$$

que é uma palavra em $W_{i}$. Os outros casos são de raciocínio análogo, usando as outras relações de Tipo 3 que encontramos para $B_{n}(M)$.

Se y é uma letra positiva, esta expressão é exatamente uma relação de Tipo 3. Pode acontecer que em $Z$ exista uma letra da forma $T_{l, n}^{ \pm 1}, \quad(l \leq i)$, mas podemos substituí-la por uma palavra em $W_{i}$ usando a relação ( $\left.P R 8\right)$. Se y é uma letra negativa, podemos deduzir a expressão acima do mesmo modo que fizemos para as relações de Tipo 3. Então podemos supor conhecidas todas as expressões acima (existe apenas um número finitos delas).

Agora, antes de começarmos com o método de pentear tranças, vamos substituir em $\bar{w}$ todas as letras da forma $a_{n, r}^{ \pm 1}$ usando a fórmula na apresentação 2

$$
a_{n, r}^{(-1)^{r+1}}=\left(A_{n, 1} A_{n, 2}^{-1} A_{n, 3} \ldots A_{n, r-1}^{ \pm 1}\right)\left(A_{n, r+1}^{ \pm 1} \ldots A_{n, 2 g-1}^{-1} A_{n, 2 g}\right)
$$


e todas as letras da forma $T_{j, n} \pm 1$ usando a relação ( $\left.P R 8\right)$. Desta forma, obtemos $\bar{w}$ como uma palavra em $W_{n-1} \cup V_{n}$.

O primeiro passo do método de pentear tranças consiste em mover para o lado direito de $\bar{w}$ todas as letras em $V_{n}$. Isto pode ser feito usando as expressões $y x y^{-1}=Z$ mencionadas acima. Deste modo, obtemos $\bar{w}=X Y$, onde $X$ é uma palavra em $W_{n-1}$ e $Y$ é uma palavra em $V_{n}$.

Agora, vamos precisar do seguinte: para o grupo fundamental de $M$, vamos denotar por $S_{n, r}$ a $n$-ésima corda de $A_{n, r}$. Desde que $s_{n, 1}, \ldots, s_{n, g}$ geram $\pi_{1}\left(M, P_{n}\right)$, o Lema 5.4.1 implica que $\left\{S_{n, 1}, \ldots, S_{n, 2 g}\right\}$ será um novo conjunto de geradores para $\pi_{1}\left(M, P_{n}\right)$. Além disso, aplicando a fórmula do Lema 5.4.1, teremos:

$$
s_{n, 1}^{-1} s_{n, 2}^{-1} \ldots s_{n, 2 g}^{-1} s_{n, 1} s_{n, 2} \ldots s_{n, 2 g}=\left(S_{n, 2 g}^{-1} S_{n, 2 g-1} S_{n, 2 g-2}^{-1} \ldots S_{n, 1}\right)\left(S_{n, 2 g} S_{n, 2 g-1}^{-1} S_{n, 2 g-2} \ldots S_{n, 1}^{-1}\right) .
$$

Então, obtemos:

$\pi_{1}\left(M, P_{n}\right)=<\left\{S_{n, 1}, \ldots, S_{n, 2 g}\right\} ;\left(S_{n, 2 g}^{-1} S_{n, 2 g-1} S_{n, 2 g-2}^{-1} \ldots S_{n, 1}\right)\left(S_{n, 2 g} S_{n, 2 g-1}^{-1} S_{n, 2 g-2} \ldots S_{n, 1}^{-1}\right)=1>$

Consideremos então a seguinte sequência exata (vide [B]):

$$
1 \longrightarrow P B_{n-1}\left(M \backslash\left\{P_{n}\right\}\right) \stackrel{u}{\longrightarrow} P B_{n}(M) \stackrel{v}{\longrightarrow} \pi_{1}\left(M, P_{n}\right) \longrightarrow 1
$$

onde para todo $\Gamma=\left(\gamma_{1}, \ldots, \gamma_{n}\right) \in P B_{n}(M), v(\Gamma)=\gamma_{n}$. Note que $v(\bar{w})=Y \in \pi_{1}(M)$. Agora, em $\pi_{1}(M)$ existe o algoritmo de Dehn que discutimos no Capítulo 1 (vide [LS]) para obtermos uma formal normal de $Y$. Para cada passo do algoritmo de Dehn, uma subpalavra de $Y$ é substituída por uma mais curta, usando a relação

$$
\left(S_{n, 2 g}^{-1} S_{n, 2 g-1} S_{n, 2 g-2}^{-1} \ldots S_{n, 1}\right)\left(S_{n, 2 g} S_{n, 2 g-1}^{-1} S_{n, 2 g-2} \ldots S_{n, 1}^{-1}\right)=1
$$

Em vez disso, faremos um processo similar em $P B_{n}(M)$ : cada vez que o algoritmo de Dehn substituir uma subpalavra de $Y$ em $\pi_{1}(M)$, nós substituiremos a correspondente subpalavra em $\bar{w}=X Y \in P B_{n}(M)$ usando a relação

$$
\left(A_{n, 2 g}^{-1} A_{n, 2 g-1} A_{n, 2 g-2}^{-1} \ldots A_{n, 1}\right)\left(A_{n, 2 g} A_{n, 2 g-1}^{-1} A_{n, 2 g-2} \ldots A_{n, 1}^{-1}\right)=\prod_{i=1}^{n-1} T_{i, n-1}^{-1} T_{i, n}
$$

a qual é equivalente a $(P R 1)$; então removemos cada $T_{i, n}^{ \pm 1}$ usando $(P R 8)$, e movemos 
novamente as letras em $V_{n}$ para o lado direito da nossa palavra.

No final deste processo, obteremos $\bar{w}=X_{n-1} w_{n}$, onde $w_{n}$ é a forma normal de $v(\bar{w})$ em $\pi_{1}(M)$, então ela é única, e $X_{n-1}$ é uma palavra em $W_{n-1}$.

O algoritmo termina em $n-1$ passos: em cada passo, temos uma palavra $X_{m}$ em $W_{m}$, substituímos as letras da forma $a_{m, r}^{ \pm 1}$ por palavras em $V_{m}$, e então, movemos como antes todas as letras em $V_{m}$ para o lado direito. Finalmente, removemos todas as subpalavras da forma $x x^{-1}$ ou $x^{-1} x$, e obtemos $X_{m}=X_{m-1} w_{m}$, onde $X_{m-1}$ é uma palavra em $W_{m-1}$ e $w_{m}$ é uma palavra reduzida em $V_{m}$. Se provarmos que a palavra $w_{m}$ é única, teremos uma única fatoração $w=w_{1} \ldots w_{n} s$ como saída do nosso algoritmo.

Definamos $M_{n-m}=M \backslash\left\{P_{m+1}, \ldots, P_{n}\right\}$ para cada $m=1, \ldots, n-1$. Em [B] encontramos a seguinte sequência exata análoga à anterior:

$$
1 \longrightarrow P B_{m-1}\left(M_{n-m+1}\right) \stackrel{f}{\longrightarrow} P B_{m}\left(M_{n-m}\right) \stackrel{g}{\longrightarrow} \pi_{1}\left(M_{n-m}\right) \longrightarrow 1 .
$$

Precisamos apenas notar que $X_{m} \in P B_{m}\left(M_{n-m}\right), g\left(X_{m}\right)=w_{m}$. Agora, desde que $\pi_{1}\left(M_{n-m}\right)$ é um grupo livre com sistema livre de geradores $\left\{a_{m, r} ; 1 \leq r \leq 2 g\right\} \cup\left\{T_{m, j} ; m+\right.$ $1 \leq j \leq n-1\}$ e desde que $w_{m}$ é uma palavra reduzida, ela é única, como queríamos.

\subsection{Apêndice}

Para finalizar nosso estudo sobre este artigo, vamos justificar agora uma questão interessante deixada pelo autor na Seção 2. A questão também é encontrada em [M], página 34, exercício 8.9:

Qual superfície orientável é representada por $\alpha_{1} \alpha_{2} \ldots \alpha_{2 g} \alpha_{1}^{-1} \alpha_{2}^{-1} \ldots \alpha_{2 g}^{-1}$ ?

Antes de justificarmos, vamos fazer uma breve referência da teoria que vamos utilizar. Esta teoria é encontrada com mais detalhes em [M], Capítulo 1.

Definição 5.7.1. Uma triangulação de uma superfície compacta $S$ consiste de uma família finita de subconjuntos fechados $\left\{T_{1}, T_{2}, \ldots, T_{n}\right\}$ que cobrem $S$ e uma familia de

homeomorfismos $\varphi_{i}: T_{i}^{\prime} \rightarrow T_{i}$, com $i=1, \ldots, n$, onde cada $T_{i}^{\prime}$ é um triângulo em $\mathbb{R}^{2}$. Cada subconjunto fechado $T_{i}$ é chamado de triângulo. Os subconjuntos de $T_{i}$ que são as imagens dos vértices e arestas do triângulo $T_{i}^{\prime}$ sob $\varphi_{i}$ são também chamados "vértices" e 
"arestas" respectivamente. Finalmente é exigido que quaisquer dois triângulos distintos $T_{i}$ e $T_{j}$ sejam distintos ou tenham somente um vértice em comum ou tenham uma aresta inteira em comum.

Definição 5.7.2. Seja $M$ uma superfície compacta com triangulação $\left\{T_{1}, T_{2}, \ldots, T_{n}\right\}$. Definimos a característica de Euler de $M$ como sendo:

$\chi(M)=\left(n^{o}\right.$. total de vértices de $\left.M\right)-\left(n^{o}\right.$. total de arestas de $\left.M\right)+\left(n^{o}\right.$. de regiões $)$.

E também, temos o seguinte resultado:

Teorema 5.7.3. Sejam $M_{1}$ e $M_{2}$ duas superfícies compactas. Então, $M_{1}$ será homeomorfa à $M_{2}$ se, e somente se, $\chi\left(M_{1}\right)=\chi\left(M_{2}\right)$ com ambas orientáveis ou ambas não orientáveis.

Consideremos também a seguinte tabela:

\begin{tabular}{|c|c|}
\hline Superfície & Característica de Euler \\
\hline \hline Esfera & 2 \\
\hline Soma conexa de $n$-toros & $2-2 n$ \\
\hline Soma conexa de $n$-planos projetivos & $2-n$ \\
\hline Soma conexa de um plano projetivo e $n$-toros & $1-2 n$ \\
\hline Soma conexa de uma garrafa de Klein e $n$-toros & $-2 n$ \\
\hline
\end{tabular}

Com isto, vamos à justificativa:

Sejam $M$ uma superfície orientável representada por $\alpha_{1} \alpha_{2} \alpha_{3} \alpha_{4} \alpha_{1}^{-1} \alpha_{2}^{-1} \alpha_{3}^{-1} \alpha_{4}^{-1}$ e o conjunto $\left\{T_{1}, T_{2}, \ldots, T_{72}\right\}$ a triangulação representada na figura a seguir:

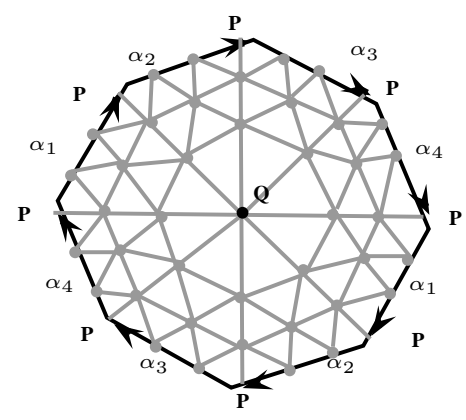


Assim, temos:

$$
\chi(M)=34-108+72=-2 .
$$

Por outro lado, pela tabela anterior sabemos que a característica de Euler da soma conexa de 2-toros é - 2. Portanto, pelo Teorema 5.7.3, $M$ é homeomorfa a soma conexa de 2-toros. Generalizando este raciocícinio, consideremos agora a superfície $M$ representada por $\alpha_{1} \alpha_{2} \ldots \alpha_{2 g} \alpha_{1}^{-1} \alpha_{2}^{-1} \ldots \alpha_{2 g}^{-1}$ e a triangulação análoga à anterior. Desta forma, vamos ter:

- $n^{o}$. de vértices: $9+(2 g-1) \cdot 5+(2 g-1) \cdot 3+1=16 g+2$.

- $n^{o}$. de arestas: $18+(2 g-1) \cdot 15+(2 g-1) \cdot 12+9=54 g$.

- $n^{o}$. de regiões: $4 g \cdot 9=36 g$.

Logo, $\chi(M)=2-2 g$ e, pela tabela anterior sabemos que a característica de Euler da soma conexa de $g$-toros é $2-2 g$. Logo, pelo Teorema 5.7.3, $M$ é homeomorfo a soma conexa de $g$-toros.

Vamos observar que precisávamos desta justificativa para trabalharmos com a representação tomada pelo autor deste artigo, já que esta representação não é a representação padrão que estudamos no Capítulo 3 da soma conexa de $g$-toros. Desta forma, mostramos que é possível usá-la já que elas são homeomorfas. 


\section{Índice Remissivo}

ação de grupos, 20

Algoritmo de Dehn, 18

característica de Euler, 116

cisão, 26

conjunto de relatores, 11

conjunto recursivo, 17

conjunto simetrizado, 17

diagrama comutativo, 22

extenção cindida, 26]

extensão de grupos, 21

família de geradores, 11

fecho normal, 11

grupo das tranças no disco

puras, 41

grupo de tranças de superfícies, 46

puras, 46

grupo de tranças no disco, 41

grupo livre, 3, 8

posto, 8

base, 8

letra, 5 monóide livre, 4

segmento, 5

segmento final, [5]

segmento inicial, 5

segmento próprio, 5

palavra, 5

ciclicamente reduzida, 9

comprimento, 5

permutação cíclica, 9

reduzida, 5

reduzida como escrito, 9

polígono, 50

inicial, 50

problema da palavra, 17

produto livre, 14

amalgamado, 16

produto semidireto, 20

push-out, 16

redução, 囵

elementar, 5 ]

relação, 11

relações definidoras, 12

relatores definidores, 12 
retração, 29

retrato, 29

por deformação, 29

sequência

exata, 22

exata curta, 22

trança geométrica, 38

elementar, 39

em superfícies, 45

tranças equivalentes, 38

em superfícies, 46

Transformações de Tietze, 13

Tipo I, 13

Tipo II, 14

triangulação de uma superfície compacta, 116 


\section{Referências Bibliográficas}

[B] J. S. Birman, Braids, Links and Mapping class Groups, Annals of Math. Studies 82 (Princeton University Press, 1973).

[B2] G. Bredon, Topology and Geometry, (Springer- Verlag New York Inc., 1993).

[BZ] G. Burde and H. Zieschang, Knots, de Gruyter Studies in Mathematics, 5 (Walter de Gruyter and Co., 1985).

[C] D. E. Cohen, Combinatorial Group Theory: A Topological Aproach, (Cambridge University Press, 1989).

[FvB] E. Fadell and J. Van Buskirk, "The braid groups of $E^{2}$ and $S^{2}$ ", Duke Math J. 29 (1962) $243-258$.

[GM] J. González-Meneses, "New Presentation of Surface Braid Groups", (Journal of Knot Theory and Its Ramifications, Vol. 10, $\left.\mathrm{n}^{\circ} .3,2001,431-451\right)$.

[H] P. De La Harpe, "An invitation to Coxeter Groups", Group theory from a geometrical viewpoint (eds E. Ghys, Haefliger and Verjovsky)(World Scientific Publishers, Singapore, 1991).

[J] D. L. Johnson, Presentation of Groups, 2nd ed., (Cambridge University Press, 1997).

[LH] V. L. Hansen, Braids and Coverings: Selected Topics, (Cambridge University Press, 1989). 
[LS] R. C. Lyndon and P. E. Schupp, Combinatorial Group Theory (Springer- Verlag, $1977)$.

[M] W. S. Massey, Algebraic Topology: An Introduction (Harcourt, Brace and World Inc., 1967).

[PR] L. Paris and D. Rolfsen, "Geometric Subgroups of Surface Braid Groups", Ann. Inst. Fourier, Grenoble, (2) 49 (1999) $101-156$.

[vB] J. Van Buskirk, "Braid Groups of Compact 2-Manifolds with Elements with Finite Order", Trans. Amer. Math. Soc. 122 (1966) 81 - 97. 